DOI 10.4171/JEMS/285

Olivier Biquard · Rafe Mazzeo

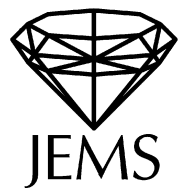

\title{
A nonlinear Poisson transform for Einstein metrics on product spaces
}

Received July 14, 2008 and in revised form September 12, 2010

\begin{abstract}
We consider the Einstein deformations of the reducible rank two symmetric spaces of noncompact type. If $M$ is the product of any two real, complex, quaternionic or octonionic hyperbolic spaces, we prove that the family of nearby Einstein metrics is parametrized by certain new geometric structures on the Furstenberg boundary of $M$.
\end{abstract}

\section{Introduction}

This paper is the first in a series to investigate the deformation theory of Einstein metrics asymptotically modelled by Riemannian globally symmetric spaces of noncompact type and of arbitrary rank. In the special case of real hyperbolic space, and the slightly more general setting of conformally compact asymptotically hyperbolic manifolds, this has been the focus of extensive study over the last fifteen years; this attention is due both to the many deep connections with conformal geometry (cf. [10]), as well as the central role that these 'Poincaré-Einstein' spaces play in the AdS/CFT correspondence in string theory, for which the proceedings [4] provide a good introduction. More recently, some of this analysis has been extended to other rank one noncompact symmetric spaces [2]. Some recent advances in linear analysis on symmetric spaces has now made it reasonable to attack this problem in greater generality.

The rank one globally symmetric spaces of noncompact type are the real, complex, and quaternionic hyperbolic spaces, denoted $\mathbb{R} H^{m}, \mathbb{C} H^{m}, \mathbb{H} H^{m}$, respectively, and the octonionic hyperbolic plane $\mathbb{O} H^{2}$. Each has curvature bounded between two negative constants and is diffeomorphic to an open ball $B^{n+1}$. There is a family of 'asymptotically $\mathbb{K}$ hyperbolic' $(\mathbb{K}=\mathbb{R}, \mathbb{C}, \mathbb{H}$ or $\mathbb{O}$ )—or briefly, A $\mathbb{K H}$-metrics, with asymptotics modelled on $\mathbb{K} H^{m}$, each of which induces a geometric structure on the sphere at infinity, $S^{n}$. In the real case, this geometric structure is a conformal class; in the complex and quaternionic case, it is a CR or quaternionic contact structure, as described in [2]. There

O. Biquard: Département de Mathématiques et Applications, École Normale Supérieure, 45 rue d'Ulm, 75005 Paris, France; e-mail: olivier.biquard@ens.fr

R. Mazzeo: Department of Mathematics, Stanford University, Stanford, CA 94305, USA; e-mail:mazzeo@math.stanford.edu

Mathematics Subject Classification (2010): Primary 53C25; Secondary 58D27 
is an octonionic contact structure on $S^{15}$, but it is rigid. We call these boundary structures either the conformal infinity data of the $\mathrm{A} \mathbb{K} \mathrm{H}$ metrics, or alternatively, $G$-conformal structures, where $G$ is the semisimple Lie group associated to $\mathbb{K} H^{m}$. One main result of [2] is that for each $G$-conformal structure near the standard one on $S^{n}$ there is a unique $\mathrm{A} \mathbb{K} \mathrm{H}$ Einstein metric near to $\mathbb{K} H^{m}$, and with that conformal infinity data. This correspondence between AKKH Einstein metrics and $G$-conformal structures can be regarded as an asymptotic boundary problem, and the assignment of the interior Einstein metric to the conformal infinity data a sort of nonlinear Poisson transform. There are other very interesting, and more subtle, problems of this type: we mention in particular LeBrun's positive frequency conjecture, concerning self-dual and anti-self-dual Einstein metrics in four dimensions, which was solved by the first author in [3].

It is our goal in this paper to establish a similar local deformation theory in the first higher rank case, namely for products of the various hyperbolic spaces listed above. There are a number of new and interesting features not encountered in the rank one case, and the details of the geometry and analysis are already sufficiently complicated that it has seemed reasonable to keep this as a separate paper. In later papers we shall treat the cases corresponding to more general noncompact higher rank symmetric spaces. This mirrors the recent developments for the linear analysis (for the scalar Laplacian) [21-24].

At the roughest level, the proof proceeds exactly as in the AKKH setting, by constructing a family of approximate Einstein metrics, parametrized by a family of boundary structures generalizing the $G$-conformal structures, and then applying the inverse function theorem to an appropriately gauged version of the Einstein operator. The solution of the resulting nonlinear elliptic equation yields the 'near-product hyperbolic' Einstein metric with the prescribed conformal infinity data. There are three main new issues in carrying this out for higher rank symmetric spaces. The first, purely geometric in nature, involves defining the appropriate analogue of $G$-conformal structures. This relies in turn on a choice of compactification for each of these product hyperbolic spaces as a manifold with corners of codimension two; the new boundary structures are defined on the corner. However, not every one of these new boundary structures can be extended to an asymptotically product hyperbolic metric which is also asymptotically Einstein everywhere near infinity. Extra hypotheses on the boundary structure must be imposed, and even then one must solve an extra Einstein-like equation to be able to extend this structure from the corner to the codimension one boundary faces. The final issue is to attain some understanding of the mapping properties of the linearized gauged Einstein operator on these asymptotically product hyperbolic metrics so that we can apply an inverse function theorem argument.

In slightly more detail, if $M_{j}$ is a rank one hyperbolic space, $j=1,2$, then the correct notion of conformal infinity data on $M_{1} \times M_{2}$ is what we call a $\left(G_{1} \times G_{2}\right)$-conformal structure on the Furstenberg boundary $\partial M_{1} \times \partial M_{2}$. This is defined carefully in $\S 5$. To construct an approximate Einstein metric associated to one of these, the first step is to extend this structure from the corner to the full boundary $\left(M_{1} \times \partial M_{2}\right) \cup\left(\partial M_{1} \times M_{2}\right)$; this involves solving an auxiliary PDE on these hypersurface boundaries, which is a coupled version of the Einstein equation on each factor $M_{j}$. Once this has been done, we can extend the conformal infinity data to an approximate solution of the problem, i.e. a 
metric which induces this designated structure on the Furstenberg boundary, and which is asymptotically Einstein uniformly near infinity. The next step is to determine the mapping properties of the linearized gauged Einstein operator on weighted Hölder spaces in order to perturb this asymptotically Einstein metric to an exact one. This is done using the geometric parametrix approach developed by the second author and Vasy, as referenced above, which involves an adaptation of the techniques of $N$-body scattering theory to study elliptic theory on symmetric spaces of rank greater than one. A subtle but important complication is that if either of the factors is quaternionic, then the approximate Einstein metrics corresponding to different $\left(G_{1} \times G_{2}\right)$-conformal structures are not mutually quasi-isometric, even up to diffeomorphism. Because of this, even the function spaces vary in a nontrivial way as we vary the boundary $\left(G_{1} \times G_{2}\right)$-conformal structure. This means that we must analyze the Green function for the linearized gauged Einstein operator not just at the exact product metric, but for all nearby approximate Einstein metrics. This necessitates that we carry out a parametrix construction at 'near-product hyperbolic metrics', which is more complicated than doing it just at the product hyperbolic space only. (This difficulty is already present in the quaternionic hyperbolic case [2]; there is a way to circumvent it then which unfortunately does not generalize to this product setting, so the parametrix construction seems unavoidable here.)

General information about the geometric analysis behind the deformation theory for Einstein metrics, particularly in the compact setting, can be found in [1]. We follow a slightly different route developed in [2]. Suppose that $g$ is Einstein, i.e. $\operatorname{Ric}^{g}-\lambda g=0$ for some real number $\lambda$. If $h$ is a sufficiently small symmetric 2-tensor, then to make the equation $h \mapsto \mathrm{Ric}^{g+h}-\lambda(g+h)$ elliptic we supplement it with the so-called Bianchi gauge condition $B^{g}(h):=\delta^{g} h+\frac{1}{2} d \operatorname{Tr}^{g} h=0$. Equivalently, we look for solutions of the nonlinear elliptic system

$$
N^{g}(h):=\operatorname{Ric}^{g+h}-\lambda(g+h)+\left(\delta^{g+h}\right)^{*} B^{g}(h)=0 .
$$

It is not hard to show that if $\lambda<0$ and $B^{g}(h) \rightarrow 0$ at infinity, then solutions of this equation correspond to Einstein metrics $g+h$ in Bianchi gauge with respect to $g$. One advantage of this gauge is that the linearization of $N^{g}$ at $h=0$ is the particularly simple operator

$$
L^{g}=\frac{1}{2}\left(\nabla^{*} \nabla-2 \stackrel{\circ}{R}\right)
$$

where the final term on the right is the usual action of the full curvature tensor for $g$ on symmetric 2-tensors. Throughout this paper, this operator will be called the linearized gauged Einstein operator.

Our principal result is

Main Theorem. Let $\left(M_{j}, g_{j}\right)$ be an $A \mathbb{K}_{j} H$ Einstein space, $j=1,2$, and let $\mathfrak{c}$ be the product $\left(G_{1} \times G_{2}\right)$-conformal structure on $\partial M_{1} \times \partial M_{2}$. Assume that 0 is not an $L^{2}$ eigenvalue for the linearized gauged Einstein operator on either $\left(M_{j}, g_{j}\right), j=1,2$, or $\left(M_{1} \times M_{2}, g_{1}+g_{2}\right)$. Let $\mathfrak{c}^{\prime}$ be any other smooth $\left(G_{1} \times G_{2}\right)$-conformal structure which is sufficiently close to $\mathfrak{c}$ in the $\mathscr{C}^{2, \alpha}$ norm. Assume also that $\mathfrak{c}^{\prime}$ satisfies the global integrability hypothesis in Definition 26. Then there is a near-product hyperbolic Einstein metric $g^{\prime}$ 
with conformal infinity data $\mathfrak{c}^{\prime}$, and moreover $g^{\prime}$ is unique amongst such metrics in a neighbourhood of the product metric $g$.

The hypothesis on the linearized gauged Einstein operator is satisfied in many situations, in particular for convex cocompact quotients of $\mathbb{K}$ hyperbolic spaces and negatively curved AKKH Einstein spaces.

We note also that the techniques and results of this paper apply somewhat more generally than when $M$ is globally a product. It would not be too difficult to define a class of manifolds and metrics with appropriate local product conditions near the corners and boundary faces to which this deformation theory also applies. As a very simple example, $M$ might be obtained by a compact topological perturbation from the product $M_{1} \times M_{2}$. However, we do not currently know any manifolds of this type which are Einstein, and so have not formulated our main result in this greater generality.

One final comment about notation. We shall be using various classes of Hölder spaces, often weighted by powers of boundary defining functions. We typically write $\mathscr{C}^{k, \alpha}$ for

Hölder spaces on compact manifolds (or in any compact set), and $\Lambda_{g}^{k, \alpha}$ when these spaces are defined relative to some complete metric $g$ on a manifold. (In fact, the subscript $g$ is replaced by some moniker for a general class of complete metrics with a type of prescribed asymptotic geometry.)

The plan of this paper is as follows. In $\S 2$ we review the geometry of AKKH hyperbolic spaces. $\$ 3$ contains a lengthy review of the geometric parametrix theory used to study elliptic theory on these spaces, which is called the $\mathbb{K} \Theta$ pseudodifferential calculus; we also establish some results here about the resolvent family of $L^{g}$ for such a metric. $\$ 4$ reviews the analysis needed to carry out the deformation theory of Einstein metrics in the AIKH setting. $\$ 5$ develops the notion of $\left(G_{1} \times G_{2}\right)$-conformal structures and some geometric properties and estimates for the corresponding asymptotically product hyperbolic metrics. The extension of these structures to the codimension one boundary faces is the subject of $\S 6$. The parametrix construction in the near-product hyperbolic case is the topic of $\S 7$, and finally, the brief $\S 8$ finishes the proof of the main theorem.

\section{Asymptotically $\mathbb{K}$ hyperbolic spaces}

This section reviews the geometry of rank one symmetric spaces of noncompact type, and of the more general class of Riemannian manifolds which are asymptotically modelled on these.

\section{Hyperbolic spaces and their conformal infinities}

The noncompact symmetric spaces of rank one are commonly called hyperbolic spaces and written as $\mathbb{K} H^{m}$, where $\mathbb{K}=\mathbb{R}, \mathbb{C}, \mathbb{H}$ (the quaternions) or $\mathbb{O}$ (the octonions). Note that $\mathbb{O} H^{m}$ exists only when $m=1,2$, and in fact $\mathbb{O} H^{1}=\mathbb{R} H^{8}$, so the only new space in this last family is the 16-dimensional octonionic hyperbolic plane $\mathbb{O} H^{2}$. As a homogeneous space, $\mathbb{K} H^{m}=G / K$, where $G$ is a real semisimple Lie group and $K$ a maximal compact 
subgroup; more specifically,

$$
\begin{array}{ll}
\mathbb{R} H^{m}=\mathrm{SO}_{1, m} / \mathrm{SO}_{m}, & \mathbb{C} H^{m}=\mathrm{SU}_{1, m} / \mathrm{U}_{m}, \\
\mathbb{H} H^{m}=\mathrm{Sp}_{1, m} / \mathrm{Sp}_{1} \mathrm{Sp}_{m}, & \mathbb{O} H^{2}=\mathrm{F}_{4}^{-20} / \mathrm{Spin}_{9} .
\end{array}
$$

These are the noncompact duals of the corresponding projective spaces $\mathbb{K} P^{m}$.

Throughout this paper we write

$$
d=\operatorname{dim}_{\mathbb{R}} \mathbb{K},
$$

so that

$$
\operatorname{dim}_{\mathbb{R}} \mathbb{K} H^{m}=m d:=n+1,
$$

where this last equality defines $n$ in terms of $m$ and $d$.

The polar coordinate expression for the metric on $\mathbb{R} H^{m}$ is

$$
g=d r^{2}+\sinh ^{2}(r) \gamma,
$$

where $\gamma$ is the standard metric on $S^{n}$. For the analogous expression on the other hyperbolic spaces, denote by $\eta \in \Omega^{1}\left(S^{n}\right) \otimes \operatorname{Im}(\mathbb{K})$ the connection 1-form of the Hopf bundle

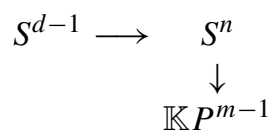

and let $\gamma$ be the pullback of the standard metric on $\mathbb{K} P^{m-1}$, regarded as a metric on the distribution $\mathscr{D}=\operatorname{ker} \eta$. The metric on $\mathbb{K} H^{m}$, normalized to have sectional curvatures in $[-4,-1]$, is given by

$$
g=d r^{2}+\sinh ^{2}(r) \gamma+\sinh ^{2}(2 r) \eta^{2} .
$$

The metric $\gamma$ on the distribution $\mathscr{D}$ can be obtained as the limit as $r \rightarrow \infty$ of the family of metrics $\gamma_{r}=\left.4 e^{-2 r} g\right|_{T S_{r}^{n}}$ on $S^{n}$; note that this limit is finite only on $\mathscr{D}$, and becomes infinite on any complementary direction. There is no natural 'origin', so $e^{-2 r}$ and $\gamma$ are defined only up to a multiplicative factor. Indeed, once we are in the fully geometric setting below, it is most natural to take $r$ as the distance from a large convex hypersurface, and then we see that $\gamma$ is only determined up to an arbitrary smooth positive factor; thus only the conformal class $[\gamma]$ of this metric on $\mathscr{D}$ is well-defined. We call this asymptotic data $(\mathscr{D},[\gamma])$ the conformal infinity of $g$.

Associated to the distribution $\mathscr{D}$ on $S^{n}$ is the bundle $\mathscr{D} \oplus\left(T S^{n} / \mathscr{D}\right)$ over $S^{n}$. The Lie bracket on sections of $\mathscr{D}$ equals $-d \eta$, and thus induces the structure of a nilpotent Lie algebra on each fibre of this extended bundle which is isomorphic to the $\mathbb{K}$-Heisenberg algebra $\mathbb{K}$ Heis $^{m-1} \cong \mathbb{K}^{m-1} \oplus \operatorname{Im}(\mathbb{K})$. The metric $\gamma$ is compatible with $d \eta \in \Omega_{\mathscr{D}}^{2} \otimes \operatorname{Im}(\mathbb{K})$ in the sense that the pair $(d \eta, \gamma)$ defines a $\mathbb{K}$-structure on $\mathscr{D}$, i.e. a collection of $d-1$ almost complex structures which are orthogonal with respect to $\gamma$, and which satisfy the algebraic relations of the basis elements in $\operatorname{Im}(\mathbb{K})$. 


\section{G-conformal structures}

The hyperbolic metrics (2.2) and (2.3) are the models for more general asymptotically hyperbolic metrics (of type $\mathbb{R}, \mathbb{C}, \mathbb{H}$ or $\mathbb{O}$ ). Before defining these, however, we first introduce terminology for the conformal infinity structures, which will also be used later in the product case.

Definition 1. Fix the $\mathbb{K}$ hyperbolic space $\mathbb{K} H^{m}=G / K$. A $G$-conformal structure on an arbitrary manifold $Y^{n}, n=m d-1$, is a codimension $d-1$ distribution $\mathscr{D} \subset T Y$, with a conformal structure $[\gamma]$ on the fibres of $\mathscr{D}$, such that the induced nilpotent Lie algebra $\mathscr{D} \oplus T Y / \mathscr{D}$ is isomorphic at each point to the $\mathbb{K}$-Heisenberg algebra, and any metric $\gamma \in[\gamma]$ is compatible with the $\mathbb{K}$ structure on $\mathscr{D}$. We say that the distribution $\mathscr{D}$ is of $\mathbb{K}$-contact type.

This definition unifies several cases:

- when $\mathbb{K}=\mathbb{R}, \mathscr{D}$ is the entire tangent space and $[\gamma]$ is a conformal structure in the usual sense;

- when $\mathbb{K}=\mathbb{C}, \mathscr{D}$ is a contact distribution in the ordinary sense; if $\eta$ is a contact 1-form which defines $\mathscr{D}$, then the compatibility of a metric $\gamma$ on $\mathscr{D}$ with $d \eta$ means that on $\mathscr{D}$ one has $d \eta(\cdot, \cdot)=\gamma(J \cdot, \cdot)$ for some almost complex structure $J$ which is orthogonal with respect to any $\gamma \in[\gamma]$; this is simply an almost CR structure on $\mathscr{D}$;

- when $\mathbb{K}=\mathbb{H}, \mathscr{D}$ is a 'quaternionic contact structure' as defined and studied in [2]; it turns out that the conformal class $[\gamma]$ is completely determined by $d \eta$;

- finally, the octonionic case is rigid; $\mathscr{D}$ is automatically locally isomorphic to the standard distribution on the sphere $S^{15}$, and $[\gamma]$ is determined completely by $d \eta$.

It is important to note here that in the quaternionic case, even though each of the tangent nilpotent Lie algebras is isomorphic to the standard quaternion Heisenberg algebra, the distribution $\mathscr{D}$ is not locally diffeomorphic to the model structure on the sphere (unless it is standard everywhere). In other words, there is no direct analogue of Darboux's theorem for quaternionic contact structures, and the infinitesimal equivalence of these structures at each point does not imply their local equivalence.

\section{A $\mathbb{K} H$ metrics}

Let $M$ be a manifold with boundary $Y=\partial M$ admitting a $G$-contact structure (associated to $\left.\mathbb{K} H^{m}=G / K\right)$. We give two equivalent definitions of the class of complete metrics on the interior of $M$ which induce a $G$-conformal structure on $Y$.

The first mimics the polar coordinate definition of the model case.

Definition 2. A metric $g$ on $M^{n+1}$ is called asymptotically $\mathbb{K}$ hyperbolic (or A $\mathbb{K} H$ for short) if the following conditions are satisfied: there is a neighbourhood $\mathscr{U}$ of $Y$ in $M$, a diffeomorphism identifying $\mathscr{U}$ with $(1, \infty)_{r} \times Y$, and a $G$-conformal structure $(\mathscr{D},[\gamma])$ on $Y$, such that, fixing a representative $(\eta, \gamma)$ of the $G$-conformal structure and defining

$$
g_{0}(\gamma, \eta)=d r^{2}+\sinh ^{2}(r) \gamma+\sinh ^{2}(2 r) \eta^{2}
$$


in $\mathscr{U}$, we have

$$
g=g_{0}(\gamma, \eta)+k
$$

where $k$ is in the weighted geometric Hölder space $e^{-v r} \Lambda_{g_{0}}^{2, \alpha}$ for some $v>0$. (The derivatives and norms are taken with respect to $g_{0}(\gamma, \eta)$.)

This definition does not depend on the choice of $(\eta, \gamma)$ in the conformal class since, replacing $(\eta, \gamma)$ by $(f \eta, f \gamma)$ for some $f \in \mathscr{C}^{\infty}(\bar{M}), f>0$, changes the model (up to diffeomorphism) by an error which is $\mathscr{O}\left(e^{-r}\right)$. The pair $([\gamma], \eta)$ (or more properly, the triple $(\mathscr{D},[\gamma], \eta))$ is called the conformal infinity of $g$.

The alternative definition simply replaces the radial variable $r$ by $x=e^{-r}$, which is a defining function for $Y$ in $M$ (recall that this means that $x \geq 0$ in $M, x=0$ only on $Y$ and $d x \neq 0$ there); thus

$$
g_{0}(\gamma, \eta)=\frac{d x^{2}+\gamma}{x^{2}}+\frac{\eta^{2}}{x^{4}}, \quad g=g_{0}(\gamma, \eta)+k,
$$

where $k \in x^{\nu} \Lambda^{2, \alpha}$. (Again, norms and derivatives are with respect to $g_{0}(\gamma, \eta)$ ). This will be more useful from our point of view since the boundary $Y$ appears explicitly as the hypersurface $\{x=0\}$. Near the boundary, the volume form of $g$ has the form

$$
d V_{g}=x^{-n-d} d x d A_{Y}(x)
$$

where $d A_{Y}(x)$ is a family of volume forms on $Y$ depending smoothly on $x$.

A straightforward calculation (cf. [2]) shows that an AKKH metric has curvature tensor which is asymptotic to that of $\mathbb{K} H^{m}$ to order $\mathscr{O}\left(e^{-v r}\right)=\mathscr{O}\left(x^{\nu}\right)$.

The complex hyperbolic metric on the unit ball $B$ in $\mathbb{C}^{m}$ has a slightly different form in standard Euclidean coordinates, and it is worth explaining the difference. This metric has Kähler form

$$
-\frac{\partial \bar{\partial} \rho}{\rho}+\frac{\partial \rho}{\rho} \wedge \frac{\bar{\partial} \rho}{\rho},
$$

where $\rho=\frac{1}{2}\left(1-|z|^{2}\right)$ is a defining function for $\partial B$. As a Hermitian metric, the first term blows up only like $1 / \rho$; its leading coefficient is the Levi form, which is positive definite on $\mathscr{D}$. The second term, which blows up at the faster rate $1 / \rho^{2}$, vanishes on (the radial extension of) $\mathscr{D}$, and is positive in the directions spanned by $\partial \rho$ and $\bar{\partial} \rho$, or equivalently, on the span of $\partial_{\rho}$ and $i \partial_{\rho}$. There are analogous expressions for the Bergman and KählerEinstein metrics on any strictly pseudoconvex domain.

The obvious discrepancy with (2.4) is resolved by setting $x=\sqrt{\rho}$. This accords with the fact that the geodesic distance function $r$ for the hyperbolic metric is comparable to $-\frac{1}{2} \log \rho$ rather than $-\log \rho$. More bluntly, the standard $\mathscr{C}^{\infty}$ structure on the closure of the Euclidean ball (or any strictly pseudoconvex domain) induced from its inclusion in $\mathbb{C}^{m}$ is not quite the right one for our purposes. 


\section{Linear elliptic theory on asymptotically hyperbolic spaces}

We now describe the structure of the Green function and mapping properties for the linearized gauged Einstein operator on an asymptotically $\mathbb{K}$ hyperbolic space.

There are several ways to approach linear elliptic problems of this type. Because the underlying geometric structure is asymptotically rank one, certain features of the operators in question are dominated by their radial behaviour, which is one-dimensional. Using this, the first author [2] carried out a detailed ODE analysis for the radial part of the relevant operators on each $\mathbb{K}$ hyperbolic space to capture the decay of the corresponding Green functions, from which the required mapping properties can be deduced. For higher rank geometries, the radial parts of these operators are multi-dimensional and must be studied using more powerful techniques. This will be done here via the machinery of geometric microlocal analysis. We begin by reviewing these methods in the setting of asymptotically $\mathbb{K}$ hyperbolic geometry, even though as we have just indicated, simpler methods are available there, because this is a good warm-up for the construction in the product case below, but also since we require in the product analysis of $\$ 7$ a number of subtle estimates on the resolvent family which do not seem to be easy to obtain in other ways.

Before embarking on all of this, we owe the reader a few words about the general strategy. Local elliptic theory (and hence global elliptic on compact manifolds) can be developed entirely via Schauder estimates. An understanding of the global mapping properties for a Laplace-type operator $L$ on a complete noncompact manifold, however, requires both this local theory and also some information about 'far-field' effects. Roughly speaking, one needs estimates at infinity for solutions of $L u=f$, even when $f \in \mathscr{C}_{0}^{\infty}$. If $L$ is invertible on $L^{2}$, for example, and we represent its inverse by an integral operator $f \mapsto u(z)=\int G\left(z, z^{\prime}\right) f\left(z^{\prime}\right) d z^{\prime}$, then $G\left(z, z^{\prime}\right)$ is a distribution on $M \times M$ usually called the Green function for $L$. Its structure near the diagonal $\left\{z=z^{\prime}\right\}$ is exactly the same as in the compact case, but the important point is to determine its asymptotics as $\left(z, z^{\prime}\right) \rightarrow \infty$ in any direction in $M \times M$. These determine the mapping properties of $L$ : for example, for nonlinear problems it is usually more convenient to use the invertibility of $L$ on Hölder spaces rather than Sobolev spaces. It is not straightforward to pass from an $L^{2}$ to a Hölder setting in general, but such things can be deduced from pointwise estimates for the offdiagonal asymptotics of $G$. Thus it is a fundamental goal to determine these asymptotics when possible.

One often deduces the existence of $G$ via Hilbert space theory and a Bochner-type argument, but this gives no information about its asymptotic structure. This is where parametrix methods can be useful. To prove that $L$ is Fredholm, it suffices to construct an operator which inverts $L$ up to compact errors. A parametrix is thus an explicitly constructed approximation to a (generalized) inverse for $L$. Hopefully, the nature of its construction yields good pointwise control on the Schwartz kernel. In special geometric settings such as the ones considered here, a parametrix construction proceeds by patching together the inverses of a set of model problems to get an approximate inverse with compact error; better approximations to the inverse are obtained by an iteration process. To organize this procedure, it is useful to perform this construction on a certain com- 
pactification $\widetilde{M^{2}}$ of $M \times M$. This compactification is a manifold with corners, and the model problems appear as the induced operators on its different boundary hypersurfaces. The off-diagonal behaviour of the parametrix (and later, the Green function) is encoded by these various boundary hypersurfaces, since these correspond to various asymptotic regimes. The fine pointwise structure of a parametrix reduces to the fact that it is a conormal (or even better, polyhomogeneous conormal) distribution on $\widetilde{M^{2}}$. A posteriori one deduces this same regularity structure for the Green function itself. The technicalities of this construction involve defining a pseudodifferential calculus on $M$ large enough to allow for a parametrix construction for 'fully elliptic' operators. Elements of such a calculus are characterized by the regularity properties of their Schwartz kernels on $\widetilde{M^{2}}$, and the main work consists in verifying the usual properties, i.e., composition, boundedness, etc. This has been carried out for a number of 'asymptotically regular' geometries. In this section we explain how this looks for the asymptotically $\mathbb{K}$ hyperbolic geometries.

We assume in this section that $L$ is a generalized Laplacian acting between sections of bundles $E$ and $F$ over $M$ associated to the bundle of orthonormal frames via a representation of the orthogonal group; these are equipped with the Levi-Civita connection $\nabla$. A geometric differential operator is a linear combination of powers $\nabla^{k}$, with coefficients determined by the metric and curvature tensor. In particular a generalized Laplacian is an operator of the form

$$
L=\nabla^{*} \nabla+R
$$

where $R$ is a symmetric endomorphism on $E$ constructed from the curvature tensor associated to $\nabla$. (Everything here adapts easily to first order Dirac-type operators, and to many other operators as well.) We shall describe the construction of a parametrix for $L$, and explain how it gives information about the Green function and mapping properties of $L$ on various function spaces. At the end of this section we collect additional facts about the resolvent family $R(\lambda)=(L-\lambda)^{-1}$ needed later.

We conclude with some historical remarks. The analysis of elliptic uniformly degenerate operators (which is the case $\mathbb{K}=\mathbb{R}$ ) appeared in [17] and [18]. The complex case was developed initially by Epstein, Melrose and Mendoza [9] (and further development and ramifications of this theory are contained in the unpublished manuscript [8]). The quaternionic and octonionic cases have not previously been written down explicitly before, though these are direct adaptations of [9]. These various pseudodifferential calculi are quite similar to one another. (For $\mathbb{K}=\mathbb{H}$, one needs to make minor adjustments because of the lack of Darboux theorem then, but this is not serious since these constructions depend on the infinitesimal, rather than the local, identifications between manifolds with $\mathbb{K} \Theta$ structure and the $\mathbb{K}$-Heisenberg models.)

\section{$\mathbb{K} \Theta$ structures}

We first describe the notion of a $\mathbb{K} \Theta$ structure on a manifold with boundary $M$, and its ancillaries: the $\mathbb{K} \Theta$ tangent, cotangent and tensor bundles, and the classes of $\mathbb{K} \Theta$ metrics and $\mathbb{K} \Theta$ differential operators, the last one containing all geometric elliptic operators for any $\mathbb{K} \Theta$ metric as elliptic elements. 
Suppose that $Y=\partial M$ carries a distribution $\mathscr{D}$ of type $\mathbb{K}$; let $([\gamma], \eta)$ be any associated conformal infinity. Choosing an identification of a neighbourhood $\mathscr{U}$ of $Y$ in $M$ as a product $Y \times[0,1)_{x}$, we extend this data to $\mathscr{U}$, and hence write down the model AKH metric $g_{0}=g_{0}(\gamma, \eta)$ as in (2.4). Now define the space $\mathscr{V}_{\mathbb{K} \Theta}$ of all smooth vector fields $V$ on the (closed) manifold $M$ such that $g_{0}(V, V)$ is smooth (in $\mathscr{U}$ ) up to $x=0$. This is independent of $\gamma$ and $\eta$, but depends on $\mathscr{D}$ (and the 1 -jet of its extension to the interior).

It is helpful to write this out in a local frame. First choose a local frame $Y_{1}, \ldots, Y_{\ell}$, $\ell=m(d-1)$, for $\mathscr{D}$ and another set of independent vector fields $Z_{1}, \ldots, Z_{r}, r=d-1$, which are complementary to $\mathscr{D}$ at each point and tangent to each $Y \times\{x\}$; the vector field $\partial_{x}$ completes this to a full basis of sections of $T M$. Then $V \in \mathscr{V}_{\mathbb{K} \Theta}$ if and only if

$$
V=a x \partial_{x}+\sum_{j=1}^{\ell} b_{j} x Y_{j}+\sum_{k=1}^{r} c_{k} x^{2} Z_{k},
$$

where $a, b_{j}, c_{k}$ are all $\mathscr{C}^{\infty}$ up to the boundary. Hence $\mathscr{V}_{\mathbb{K} \Theta}$ is locally the span over $\mathscr{C}^{\infty}(\bar{M})$ of $\left\{x \partial_{x}, x Y_{1}, \ldots, x Y_{\ell}, x^{2} Z_{1}, \ldots, x^{2} Z_{r}\right\}$.

The term ' $\Theta$-structure' comes from [9], where $\Theta$ denotes a nonvanishing section of $T_{\partial M}^{*} M \otimes \operatorname{Im}(\mathbb{K})$, the pullback of which to $T^{*} \partial M$ equals $\eta$. Hereafter, we let $\Theta$ denote not only this form on $\partial M$, but also some choice of smooth extension to the interior. All of the notions here can be defined in terms of this form, or equivalently, the corresponding oriented $(d-1)$-plane bundle $S$ of 1 -forms on $M$ at $\partial M$. Thus, for example, elements of $\mathscr{V}_{\mathbb{K} \Theta}$ are also characterized as vector fields which are smooth on $\bar{M}$ and which satisfy $\Theta(V)=\mathscr{O}\left(x^{2}\right)$.

Note that $\mathscr{V}_{\mathbb{K} \Theta}$ is closed under Lie bracket. Next, there is a vector bundle, ${ }^{\mathbb{K} \Theta} T M$, for which $\mathscr{V}_{\mathbb{K} \Theta}$ is the entire space of smooth sections. The fibres are defined by

$$
{ }^{\mathbb{K} \Theta} T_{p} M=\mathscr{V}_{\mathbb{K} \Theta} / \mathscr{I}_{p} \mathscr{V}_{\mathbb{K} \Theta},
$$

where $\mathscr{I}_{p}$ denotes the space of smooth functions on $M$ vanishing at $p$. (For a more prosaic definition, we take the sections $x \partial_{x}, x Y_{i}, x^{2} Z_{j}$ as a local basis of sections of ${ }^{\mathbb{K} \Theta} T M$.) This bundle is naturally isomorphic to $T M$ over the interior, but the natural bundle map

$$
\mathbb{K}^{\Theta} T M \rightarrow T M
$$

defined via evaluation, $V \mapsto V(p)$, is the zero map when $p \in Y$. The subbundle over $\partial M$ spanned by $\left\{x Y_{i}, x^{2} Z_{j}\right\}$ can also shown to have an invariant definition, and we denote it (with a slight abuse of notation) by ${ }^{\mathbb{K} \Theta} T \partial M$. Third, when $p \in Y$, the subspace $\mathscr{I}_{p} \mathscr{V}_{\mathbb{K} \Theta}$ is an ideal in $\mathscr{V}_{\mathbb{K} \Theta}$ with respect to the bracket of vector fields; hence for such $p,{ }^{\mathbb{K} \Theta} T_{p} M$ is a Lie algebra, where the Lie bracket of two elements given as the equivalence class of the vector field bracket of representatives of the two individual classes, and as such is isomorphic to the solvable homogeneous extension $\mathbb{K} \mathscr{S}^{m}$ of $\mathbb{K} \mathrm{Heis}^{m-1}$, the $\mathbb{K}$-Heisenberg algebra. Note that ${ }^{\mathbb{K} \Theta} T_{p} \partial M$ is a nilpotent subalgebra, isomorphic to $\mathbb{K}$ Heis $^{m-1}$ itself.

In the simplest case, when $\mathbb{K}=\mathbb{R}$, this space of vector fields is usually called the space of uniformly degenerate vector fields, denoted $\mathscr{V}_{0}$, and consists of all smooth vector fields on $\bar{M}$ vanishing at $Y$. There is no form $\Theta$ now, so to avoid complicating the presentation 
we shall mostly discuss only the other cases, save for a few passing comments about the real case. The other familiar case is when $M$ is a strictly pseudoconvex domain in $\mathbb{C}^{m}$; the $\mathbb{C} \Theta$ structure is the $\mathrm{CR}$ structure on the boundary and the canonical Bergman or KählerEinstein metrics are $\mathbb{C} \Theta$ metrics (admittedly only polyhomogeneous rather than $\mathscr{C}^{\infty}$ ). As explained earlier, one needs to take $x$ as the square root of the Euclidean distance to the boundary in order to fit this into the present framework.

The dual of the $\mathbb{K} \Theta$ tangent bundle is denoted ${ }^{\mathbb{K} \Theta} T^{*} M$. Note that smooth sections of this $\mathbb{K} \Theta$ cotangent bundle are singular in the ordinary sense: in terms of the dual basis of one-forms $d x, Y_{i}^{*}$ and $Z_{j}^{*}$,

$$
\mathscr{C}^{\infty}\left(M ;{ }^{\mathbb{\Theta}} T^{*} M\right) \ni \omega=a \frac{d x}{x}+\sum b_{i} \frac{Y_{i}^{*}}{x}+\sum c_{j} \frac{Z_{j}^{*}}{x^{2}},
$$

where $a, b_{i}, c_{j} \in \mathscr{C}^{\infty}(M)$. Similar remarks apply to all other tensor bundles too. Note in particular that an $\mathrm{A} \mathbb{K} H$ metric $g$ is a section of $S^{2}\left({ }^{\Theta} \Theta T^{*} M\right)$ which is positive definite on ${ }^{\mathbb{K} \Theta} T M$. If $E$ is any bundle constructed functorially from $T M$, then applying the same functorial operations to ${ }^{\mathbb{K} \Theta} T M$ yields a bundle which we denote ${ }^{\mathbb{K} \Theta} E$.

Definition 3. Let $M$ be a compact manifold with boundary and $\mathscr{D}$ a distribution of type $\mathbb{K}$ on $Y=\partial M$. The space Diff $\mathbb{K} \Theta_{\Theta}^{*}(M)$ of $\mathbb{K} \Theta$ differential operators on $M$ consists of all operators which can be locally expressed as a finite sum of products of elements of $\mathscr{V}_{\mathbb{K} \Theta}$. If $E, F$ are any vector bundles over $M$, then a $\mathbb{K} \Theta$ operator acting between sections of $E$ and $F$ is one which has this form with respect to any local trivialization.

It is important for $\mathscr{V}_{\mathbb{K} \Theta}$ to be closed under Lie bracket for this space of operators to be well-defined.

In our applications, the bundles $E$ and $F$ are geometric bundles, and the operator is a $\mathbb{K} \Theta$ differential operator between ${ }^{\mathbb{K} \Theta} E$ and ${ }^{\mathbb{K} \Theta} F$.

Theorem 4. Let $g$ be any $A \mathbb{K} H$ metric on $M$, and L a geometric elliptic operator of order $\mu$ between sections of two geometric bundles $E$ and $F$. Then $L \in \operatorname{Diff}_{\mathbb{K} \Theta}^{\mu}\left(M ;{ }^{K \Theta} E,{ }^{\mathbb{K} \Theta} F\right)$.

This result is tautological once one checks that the Levi-Civita connection $\nabla$ satisfies

$$
\nabla: \mathscr{C}^{\infty}(M ; E) \rightarrow \mathscr{C}^{\infty}\left(M ; E \otimes \mathbb{K}^{\Theta} T^{*} M\right) .
$$

We leave the details to the reader.

There is a principal symbol mapping for $\mathscr{V}_{\mathbb{K} \Theta}$ operators, defined by formally replacing $x \partial_{x}, x Y_{i}$ and $x^{2} Z_{j}$, respectively, by linear coordinates $\xi, \eta_{i}, \zeta_{j}$. Thus,

$$
\begin{aligned}
\operatorname{Diff}_{\mathbb{K} \Theta}^{\mu}(M ; E, F) \ni P= & \sum_{j+|\alpha|+|\beta| \leq \mu} a_{j \alpha \beta}(z)\left(x \partial_{x}\right)^{j}(x Y)^{\alpha}\left(x^{2} Z\right)^{\beta} \\
& \mapsto{ }^{\mathbb{K} \Theta} \sigma_{\mu}(P)(z ; \xi, \eta, \zeta)=\sum_{j+|\alpha|+|\beta|=\mu} a_{j \alpha \beta}(z) \xi^{j} \eta^{\alpha} \zeta^{\beta}
\end{aligned}
$$

The usual calculation shows that this is a well-defined smooth function on ${ }^{\mathbb{\Theta} \Theta} T^{*} M$ (with values in $\operatorname{Hom}(E, F)$ ), homogeneous of degree $\mu$ on the fibres.

Definition 5. The operator $P \in \operatorname{Diff}_{\mathbb{K} \Theta}^{\mu}(M ; E, F)$ is called $(\mathbb{K} \Theta)$ elliptic if ${ }^{\mathbb{K} \Theta} \sigma_{\mu}(P)(z ; \xi, \eta, \zeta)$ is an invertible endomorphism whenever $(\xi, \eta, \zeta) \neq 0$. 


\section{Parabolic dilations and model operators}

The key to the analysis of $\mathbb{K} \Theta$ operators is their approximate dilation invariance. More precisely, for any $p \in Y$, one may define an equivalence class of dilations based at $p$. When $\mathbb{K}=\mathbb{R}$, these are ordinary radial dilations, but in the other cases the dilations are 'parabolic'. Using these we can define for any $P \in \operatorname{Diff}_{\mathbb{K} \Theta}^{*}$ the normal operator $N_{p}(P)$; this is a finite-dimensional reduction in that it is a left-invariant operator on the solvable group $\mathbb{K}^{m}$, depending parametrically on $p \in Y$. Its invertibility (for all $p$ ) is the other key hypothesis, besides symbol ellipticity, needed to prove that $P$ is Fredholm.

We begin by defining these families of dilations. The situation is simplest when $\mathbb{K}=\mathbb{R}$; in this case, choose a diffeomorphism of a neighbourhood of $p$ in $M$ with a half-ball around the origin in the half-space $\mathbb{R}_{+}^{n}=\left\{(s, u): s \geq 0, u \in \mathbb{R}^{n-1}\right\}$. Now use this identification and the ordinary dilation operator $M_{\delta}:(s, u) \mapsto(\delta s, \delta u)$ to define the sequence of pushforwards of the vector field $V \in \mathscr{V}_{0}$ :

$$
\lim _{\delta \rightarrow 0}\left(M_{\delta}^{-1}\right)_{*} V=N_{p}(V) .
$$

From the local coordinate description of $V$, we see readily that $N_{p}(V)$ is a left-invariant operator defined on $\mathbb{R}_{+}^{n} \cong \mathbb{R} \mathscr{S}^{n}$. More generally, for any uniformly degenerate differential operator $P$, define

$$
\lim _{\delta \rightarrow 0}\left(M_{\delta}^{-1}\right)_{*} P=N_{p}(P)
$$

this is in the universal enveloping algebra of $\mathbb{R} \mathscr{S}^{n}$, and is well-defined up to the action of an element $A \in \mathbb{R} \mathscr{S}^{n}$.

In the other cases we begin by recalling the parabolic dilation structure on $\mathbb{K}^{m}$. To define this, recall that $\mathbb{K} \mathscr{S}=\mathbb{R}^{+} \ltimes \mathbb{K}_{\text {Heis }}{ }^{m-1}$ (this is just the $A \cdot N$ part of the $G=K A N$ decomposition). Choose a system of coordinates $(s, \sigma, u)$ where $s>0$, $\sigma \in \mathbb{R}^{d-1}=\operatorname{Im} \mathbb{K}$ and $u \in \mathbb{R}^{d(m-1)}=\mathbb{K}^{m-1}$ so that (in $\mathbb{K}$ coordinates)

$$
\Theta_{0}=d \sigma+\frac{1}{2} \operatorname{Im}(d u \cdot \bar{u})
$$

defines the standard $\mathbb{K}$ contact structure on $\mathbb{K}$ Heis $^{m-1}$. Thus,

$$
s \partial_{s}, s\left(\partial_{u_{i}}-\frac{1}{2} \operatorname{Im}\left(\partial_{u_{i}} \bar{u}\right)\right), s^{2} \partial_{\sigma_{j}}
$$

is a basis of left-invariant vector fields, where in $\operatorname{Im}\left(\partial_{u_{i}} \bar{u}\right)$ we identify the vector $\partial_{u_{i}}$ with a vector with $\mathbb{K}$ coordinates in $\mathbb{K}^{m-1}$, and the imaginary quaternions with vertical vectors $\partial_{\sigma_{j}}$. The dilation is then given by

$$
M_{\delta}(s, \sigma, u)=\left(\delta s, \delta^{2} \sigma, \delta u\right) .
$$

(Note that these vector fields are homogeneous of degree 0 with respect to $M_{\delta}$.) When $\mathbb{K}=\mathbb{C}$ or $\mathbb{O}$, we can choose a diffeomorphism as before which identifies a neighbourhood of $p$ in $M$ with a neighbourhood of 0 in $\mathbb{K} \mathscr{S}$, and carries the distribution $\mathscr{D}$ to the model distribution on $\mathbb{K}$ Heis $^{m-1}$; the model $\Theta_{0}$ above is a suitable choice for $\Theta$ on $M$. In the complex case this uses the Darboux theorem, while in the octonion case this follows 
from the local rigidity of octonion contact structures (so that $(Y, \mathscr{D})$ is locally identified with the model geometry). In terms of this identification, we define $N_{p}(P)$ by the same formula as above, arriving at an operator which is left-invariant on $\mathbb{K} \mathscr{S}$ and well-defined up to translation by an element of this group. In the last case, $\mathbb{K}=\mathbb{H}$, one has no longer the Darboux theorem or rigidity, but the following lemma is proved in the Appendix.

Lemma 6. For any quaternionic contact structure on $Y^{4 m-1}$, and any point $p \in Y$, there exist local coordinates $(\sigma, u) \in \operatorname{Im}(\mathbb{H}) \times \mathbb{H}^{m-1}$ such that the quaternionic distribution is given by the kernel of a 1-form $\Theta$ with values in $\operatorname{Im}(\mathbb{H})$, and the difference with the standard form $\Theta_{0}$ of the Heisenberg group near the origin $(\sigma=0, u=0)$ satisfies the estimate $\left|\Theta-\Theta_{0}\right|=O\left(|u|^{2}+|\sigma|\right)$. The two nilpotent algebra structures coincide at the point p.

This result constructs a diffeomorphism from a neighbourhood of $p$ to a neighbourhood of 0 in $\mathbb{H} \mathscr{S}$ so that the distributions agree at the origin. Letting $\left\{Y_{i}\right\}$ and $\left\{Y_{i}^{0}\right\}$ be local frames for $\mathscr{D}$ and the model distribution (in $\mathbb{H} H e i s^{m-1}$, extended to $\mathbb{H} \mathscr{S}^{m}$ and then transferred to this neighbourhood), then clearly $Y_{i}=Y_{i}^{0}+O\left(|u|^{2}+|\sigma|\right)$. It follows that the limit of the parabolic dilations of $P$ is still a left-invariant operator on $\mathbb{H} \mathscr{S}$.

To express this more concretely, fix a boundary defining function and smooth vector fields $Y_{i}, i=1, \ldots, d(m-1), Z_{j}, j=1, \ldots, d-1$, such that the $Y_{i}$ span the extension of $\mathscr{D}$ and the $Z_{j}$ span a subspace complementary to $\mathscr{D}$ at each point. Using the obvious multi-index notation, write

$$
P=\sum_{j+|\alpha|+|\beta| \leq m} a_{j \alpha \beta}(w)\left(x \partial_{x}\right)^{j}(x Y)^{\alpha}\left(x^{2} Z\right)^{\beta},
$$

where the coefficients are assumed to be $\mathscr{C}^{\infty}$ (up to the boundary). The values of $x \partial_{x}$, $x Y_{i}$ and $x^{2} Z_{j}$ at $p$ fix an isomorphism of ${ }^{\mathbb{K} \Theta} T_{p}^{*} M$ and $\mathbb{K} \mathscr{S}$, and

$$
N_{p}(P)=\sum_{j+|\alpha|+|\beta| \leq m} a_{j \alpha \beta}(p)\left(s \partial_{s}\right)^{j}\left(s Y^{0}\right)^{\alpha}\left(s^{2} Z^{0}\right)^{\beta}
$$

where $s \in \mathbb{R}^{+}$, and $\left\{Y_{1}^{0}, \ldots, Y_{d(m-1)}^{0}, Z_{1}^{0}, \ldots, Z_{d-1}^{0}\right\}$ is a fixed basis of left-invariant vector fields on $\mathbb{K}$ Heis $^{m-1}$.

The following result is well-known in the real and complex cases. It is obvious in the octonionic case, and a direct consequence of Lemma 6 in the quaternionic case.

Proposition 7. Let $g$ be an $A \mathbb{K} H$ metric on $M$, and L be a generalized Laplace operator on $M$. Then, at each point of $\partial M$, the normal operator of $L$ identifies to the corresponding operator on the hyperbolic space $\mathbb{K} H^{m}$. In particular, up to isomorphism, it does not depend on the point of $\partial M$.

For $P$ as above, there is a simpler family of model ordinary differential operators on $\mathbb{R}^{+}$ called the indicial family, defined by the expression

$$
I_{p}(P)=\sum_{j \leq m} a_{j 00}(p)\left(s \partial_{s}\right)^{j} .
$$


The coefficients are endomorphisms of $E_{p}$. Since this is a constant coefficient Fuchsian operator, it is equivalent by Mellin transform to multiplication by a (matrix-valued) polynomial

$$
I_{p}(P ; \zeta)=\sum_{j \leq m} a_{j 00}(p)(\zeta)^{j}
$$

A number $\zeta \in \mathbb{C}$ is called an indicial root if $I_{p}(P ; \zeta)$ is singular. This is equivalent to the requirement that

$$
P\left(x^{\zeta} v(y)\right)=\mathscr{O}\left(x^{\zeta+1}\right) \quad \forall v \in \mathscr{C}^{\infty}(Y) .
$$

These indicial roots are fundamental invariants of $P$.

\section{Blowups and the $\mathbb{K} \Theta$ double space}

There is a more sophisticated way to interpret the parabolic dilations, leading to a more obviously invariant definition of normal operators. The idea is to introduce a resolution, or blowup, of the product space $M \times M$ which reflects the scaling invariance properties of $\mathbb{K} \Theta$ differential operators near the boundary. This provides the means to define the $\mathbb{K} \Theta$ pseudodifferential operators. We describe this now.

As usual, we start with the simplest case $\mathbb{K}=\mathbb{R}$. The distributional Schwartz kernels of pseudodifferential operators are singular along the diagonal in $M \times M$. Unfortunately, this diagonal intersects the corner $\partial M \times \partial M$, making it difficult to describe the precise structure of its singularity near this intersection. To remedy this we introduce a new space

$$
M_{0}^{2}=\left[M \times M ; \operatorname{diag}_{\partial M \times \partial M}\right],
$$

where the notation on the right indicates that we blow up $M \times M$ at the boundary of the diagonal. This amounts to replacing this submanifold by the space of inward pointing unit normal vectors; the space $M_{0}^{2}$ is endowed with the smallest $\mathscr{C}^{\infty}$ structure containing the lifts of all smooth functions on $M \times M$ and polar coordinates around $\operatorname{diag}_{\partial M}$. Thus $M_{0}^{2}$ has three hypersurface boundaries, $B_{10}$ and $B_{01}$, the left and right faces, which are the ones lifted from the two hypersurface boundaries in $M \times M$, and the new front face $B_{11}$ created in this blowup, which is often also denoted ff. The blowdown map $\beta: M_{0}^{2} \rightarrow M^{2}$ is a smooth mapping of manifolds with corners.

The front face $B_{11}$ fibres over $\operatorname{diag}_{\partial M}$, with fibre at $p \in Y$ the set of unit inner normal vectors at that point; this is a quarter-sphere, the interior of which carries a natural projective structure. Let $(x, y)$ and $\left(x^{\prime}, y^{\prime}\right)$ denote coordinates on the two copies of $M$ in $M^{2}$; we are blowing up the submanifold $x=x^{\prime}=0, y=y^{\prime}$, and so it is legitimate to introduce the new singular coordinate system $s=x / x^{\prime}, u=\left(y-y^{\prime}\right) / x^{\prime}, x^{\prime}, y^{\prime}$. The coordinates $(s, u)$ are then projective coordinates on this quarter-sphere. The normal operator of $P$ is the restriction to the fibres of $B_{11}$ of the lift of $P$ from the left factor of $M$ to $M^{2}$ and then to $M_{0}^{2}$. Thus, as in the previous definition, each $N_{p}(P)$ acts on a half-space $\mathbb{R}_{+}^{n}=\mathbb{R}^{+} \ltimes \mathbb{R}^{n-1}$. The underlying dilation structure is implicit here since we are taking the normal blowup, which involves ordinary homothetic scaling in the tangent spaces.

There is a similar development for the other cases (see [9] in the complex case), but the normal blowup of the boundary of the diagonal must be replaced by a blowup of this 
submanifold which respects the underlying parabolic dilation structure. Now, instead of ordinary spherical normal vectors, we use equivalence classes of paths converging to $p$, where the equivalence relationship is governed by the form $\mathbb{K} \Theta$.

First define $I_{Y}$ to consist of the smooth functions on $M$ vanishing on $Y$ and, recalling the bundle $S \subset T_{\partial M}^{*} M$ determined by $\Theta$, let $I_{S}$ denote the subset of those elements $f \in I_{Y}$ such that $\left.d f\right|_{Y}$ are sections of $S$. Next, fix $p \in Y$ and define the set of $S$-parabolic curves at $p$ to consist of those smooth functions $\gamma:[0,1] \rightarrow M$ with $\gamma(0)=p$ and $f(\gamma(t))=\mathscr{O}\left(t^{2}\right)$ for all $f \in I_{S}$. We define an equivalence relation on such curves:

$$
\begin{aligned}
\gamma_{1} \sim \gamma_{2} \Leftrightarrow & f\left(\gamma_{1}(t)\right)-f\left(\gamma_{2}(t)\right)=\mathscr{O}\left(t^{2}\right) \forall f \in I_{Y} ; \\
& f\left(\gamma_{1}(t)\right)-f\left(\gamma_{2}(t)\right)=\mathscr{O}\left(t^{3}\right) \forall f \in I_{S} .
\end{aligned}
$$

The space of equivalence classes is the set of inward-pointing $S$-parabolic normal vectors to $Y$ at $p$, which we denote $\mathscr{S}_{p}$, and these fit together to form a bundle $\mathscr{S}$ over $Y$. Each $\mathscr{S}_{p}$ has a natural $\mathbb{R}^{+}$and additive structure, defined by $\delta[\gamma(t)]=[\gamma(\delta t)]$ and $[\gamma]=\left[\gamma_{1}\right]+$ $\left[\gamma_{2}\right]$ if $f(\gamma(t))-\left(f\left(\gamma_{1}(t)\right)+f\left(\gamma_{2}(t)\right)=\mathscr{O}\left(t^{2}\right)\right.$ for all $f \in I_{Y}$, and $\mathscr{O}\left(t^{3}\right)$ for all $f \in I_{S}$, respectively. These do not define a linear structure, however, since the scalar action does not distribute over addition. However, directly from a local coordinate calculation one finds that $\mathscr{S}_{p} \cong \mathbb{K} \mathscr{S}$, and scalar multiplication corresponds to parabolic dilation.

\section{Conormal distributions}

We make a small diversion from the main thread of this section to recall the definitions of conormal and polyhomogeneous conormal distributions on manifolds with corners since these are used in many places below. Good references for this material include [18] and [25], to which we refer for more details.

Let $X$ be a manifold with corners and $\left\{H_{j}\right\}_{j=1}^{N}$ an enumeration of the boundary hypersurfaces of $X$. We assume that each $H_{j}$ is a smooth embedded submanifold with corners in $X$, so we can fix a global defining function $\rho_{j}$ for that face, i.e. $\rho_{j} \geq 0, H_{j}=\left\{\rho_{j}=0\right\}$ and $d \rho_{j} \neq 0$ there.

The space $\mathscr{V}_{b}(X)$ of $b$-vector fields on $X$ consists of all smooth vector fields $V$ which are arbitrary in the interior of $X$ and which lie tangent to all boundary faces, and hence all corners. Any point $p \in \partial X$ lies in some corner of codimension $k, H_{j_{1}}, \ldots, H_{j_{k}}$. Choose local coordinates $\left(x_{1}, \ldots, x_{k}, y\right)$ near $p$ with $x_{i}=\rho_{j_{i}}, i=1, \ldots, k$, and where $y=$ $\left(y_{1}, \ldots, y_{n-k}\right)$ lies in an open neighbourhood of 0 in $\mathbb{R}^{n-k}$. Thus near $p$, any $V \in \mathscr{V}_{b}$ can be written as

$$
V=\sum a_{i j}(x, y) x_{i} \partial_{x_{j}}+\sum b_{\ell}(x, y) \partial_{y_{\ell}}, \quad a_{i j}, b_{\ell} \in \mathscr{C}^{\infty} .
$$

In other words, $\mathscr{N}_{b}(X)$ is spanned locally over $\mathscr{C}^{\infty}(X)$ by $x_{i} \partial_{x_{j}}, \partial_{y_{\ell}}, i, j=1, \ldots, k$, $\ell=1, \ldots, n-k$.

Definition 8. For any multi-weight $\sigma=\left(\sigma_{1}, \ldots, \sigma_{N}\right) \in \mathbb{R}^{N}$, define $\rho^{\sigma}=\rho_{1}^{\sigma_{1}} \ldots \rho_{N}^{\sigma_{N}}$; then the space of conormal distributions of order $\sigma$ is

$$
\mathscr{A}^{\sigma}(X)=\left\{u: V_{1} \ldots V_{r} u \in \rho^{\sigma} L^{\infty}(X) \text { for all } r \geq 0 \text { and } V_{j} \in \mathscr{V}_{b}\right\} .
$$

We also write $\mathscr{A}(X)=\bigcup_{\sigma} \mathscr{A}^{\sigma}(X)$. 
Elements of $\mathscr{A}(X)$ are tangentially regular, and have a certain normal regularity too, but (for example) elements of $\mathscr{A}^{0}(X)$ do not necessarily have well-defined boundary values. Typical conormal distributions include $\rho^{\gamma}$ and $|\log \rho|^{s}$, where $\gamma$ and $s$ are multiindices in $\mathbb{C}$.

Polyhomogeneous functions constitute the most useful subclass of $\mathscr{A}(X)$. By definition, $u \in \mathscr{A}(X)$ is polyhomogeneous if near any point $p$ which lies in a corner of codimension $k$ in $X$, then using coordinates and multi-indices as above,

$$
u \sim \sum a_{\gamma, \ell}(y) x^{\gamma}(\log x)^{\ell}, \quad a_{\gamma, \ell} \in \mathscr{C}^{\infty} .
$$

Here $(\gamma, \ell)$ varies over a discrete set in $\mathbb{C}^{k} \times \mathbb{N}^{k}$ which has finite intersection with each sector $\bigcup_{j=1}^{k}\left\{\gamma: \operatorname{Re}\left(\gamma_{j}\right) \leq C_{j}\right\} \times \mathbb{N}^{k}$.

A $p$-submanifold $Y \subset X$ is, by definition, one which can be expressed locally in terms of adapted boundary coordinates as $\left\{x_{i_{1}}=\cdots=x_{i_{j}}=0, y_{r_{1}}=\cdots=y_{r_{s}}=0\right\}$ (the $p$ means 'product', i.e. $Y$ is locally of product form in $X$ ). One can form the blowup of any such $Y$ in $X$ to obtain a space $[X ; Y]$. A distribution is conormal or polyhomogeneous to $Y$ if its lift to $[X ; Y]$ has either of these properties at the boundary face of $[X ; Y]$ which covers $Y$.

Although the Schwartz kernels we deal with below are polyhomogeneous, we typically only use a property only slightly stronger than conormality. Fix a boundary face $H$ and suppose that the weight $\sigma_{H}$ corresponding to this face is 0 . We say that $u \in \mathscr{A}_{H}^{\sigma}(X)$ if $u \in \mathscr{A}^{\sigma}(X)$ as before, and that near $H, u=u_{0}+v$ where both $u_{0}$ and $v$ are conormal, but $u_{0}$ is smooth up to $H$ and $v$ vanishes like $\rho_{H}^{\varepsilon}$ there. In other words, $u$ decomposes into a 'leading coefficient' $u_{0}$, which is a smooth function on $H$ (conormal at all boundaries of $H$ ) and a conormal remainder term $v$ which vanishes to some positive order. If $\mathscr{H}$ is a subset of the set of all boundary faces, then $\mathscr{A}_{\mathscr{H}}^{\sigma}(X)$ consists of functions with this type of decomposition at each face $H \in \mathscr{H}$. If $Y$ is an interior $p$-submanifold of $X$, and $\mathscr{H}$ some subset of the boundary faces of $X$, all elements of which intersect $Y$ at $\partial X$, and $\sigma_{H}=0$ for all $H \in \mathscr{H}$, then we define $\mathscr{A}_{\mathscr{H}}^{\sigma}(X ; Y)$ to consist of all $u$ which can be decomposed as a sum $u^{\prime}+u^{\prime \prime}$ where $u^{\prime} \in \mathscr{A}_{\mathscr{H}}^{\sigma}(X)$ and $u^{\prime \prime}$ is supported in a small neighbourhood of $Y$, and is polyhomogeneous along $Y$ with polyhomogeneous singularity smoothly extendible across all boundary faces of $Y$.

All of these definitions generalize immediately if $u$ is a section of some smooth vector bundle over $X$.

\section{$\mathbb{K} \Theta$ pseudodifferential operators}

The $\mathbb{K} \Theta$ double space provides the geometric setting for Schwartz kernels of $\mathbb{K} \Theta$ pseudodifferential operators.

Definition 9. For any $\mu \in \mathbb{R}$ and set of weights $\sigma=\left(\sigma_{10}, \sigma_{01}, 0\right)$ corresponding to the boundary faces $B_{10}, B_{01}$ and $B_{11}$ of $M_{\mathbb{K} \Theta}^{2}$, the space $\Psi_{\mathbb{K} \Theta}^{\mu, \sigma}(M)$ of conormal $\mathbb{K} \Theta$ pseudodifferential operators on $M$ consists of all those operators $A$ on $M$ with the following properties: 
- the Schwartz kernel $K_{A}$ of $A$ is the pushforward (under the blowdown $\beta: M_{\mathbb{K} \Theta}^{2} \rightarrow M^{2}$ ) of a distribution $\kappa_{A}$ on $M_{\mathbb{K} \Theta}^{2}$;

- $\kappa_{A}$ is a distribution on $M_{\mathbb{K} \Theta}^{2}$ which is conormal with respect to all boundaries, partially homogeneous with respect to the front face, and which has a polyhomogeneous singularity of pseudodifferential order $\mu$ along the lifted diagonal $\operatorname{diag}_{\mathbb{K} \Theta}$, i.e.

$$
\kappa_{A} \in \mathscr{A}_{B_{11}}^{\sigma}\left(M_{\mathbb{K} \Theta}^{2}, \operatorname{diag}_{\mathbb{K} \Theta}\right) \text {. }
$$

Slightly more generally, we also define $\Psi_{\mathbb{K} \Theta}^{\mu, \sigma}(M)$ in an analogous way when $\sigma=$ $\left(\sigma_{10}, \sigma_{01}, \sigma_{11}\right)$ and $\sigma_{11}>0$ (however, dropping the partial polyhomogeneity at $B_{11}$ and only requiring that $\kappa_{A}$ is conormal and vanishes to order $\sigma_{11}$ at that face).

The action of $K_{A}$ on a function $f$ on $M$ requires the choice of a density $\gamma$ on $M$ against which to integrate, so that $\left(K_{A} f\right)(z)=\int_{M} K_{A}\left(z, z^{\prime}\right) f\left(z^{\prime}\right) \gamma\left(z^{\prime}\right)$. It is purely a matter of convention whether we fix $\gamma$ to be a smooth measure on $\bar{M}$, for example, or some power of a defining function times a smooth measure; any two such choices yield equivalent theories, but one does need to make an adjustment to the index set $\sigma$ below based on this choice. We shall follow the convention that $\gamma$ is instead a volume form for some fixed $\mathbb{K} \Theta$ metric, and hence is of the form $x^{-n-d}$ times a smooth measure. This has the advantage that most of the operators in our later applications are self-adjoint.

Basic facts about these spaces of pseudodifferential operators include the composition law

$$
\Psi_{\mathbb{K} \Theta}^{\mu, \sigma} \circ \Psi_{\mathbb{K} \Theta}^{\mu^{\prime}, \sigma^{\prime}} \subset \Psi_{\mathbb{K} \Theta}^{\mu+\mu^{\prime}, \sigma^{\prime \prime}}, \quad \sigma^{\prime \prime}=\left(\sigma_{10}, \sigma_{01}^{\prime}, 0\right),
$$

which holds provided $\sigma_{01}+\sigma_{10}^{\prime}>-1$ (this condition is needed to ensure that the integration defining the composition makes sense), and the existence of a short exact symbol sequence

$$
0 \rightarrow \Psi_{\mathbb{K} \Theta}^{\mu-1, \sigma} \rightarrow \Psi_{\mathbb{K} \Theta}^{\mu, \sigma} \rightarrow S^{\mu}\left({ }^{\mathbb{K} \Theta} T^{*} M\right) \rightarrow 0 .
$$

The properties of this sequence, and the existence of a quantization map from $S^{\mu}\left({ }^{\mathbb{K} \Theta} T^{*} M\right)$ to $\Psi_{\mathbb{K} \Theta}^{\mu, \sigma}$ which is well-defined modulo operators of order $\mu-1$, follows almost exactly as in the standard interior case. The composition law (3.6) is substantially more subtle. Indeed, it could be regarded as the most technically difficult part of the $\mathbb{K} \Theta$ pseudodifferential calculus. The proof is fairly involved, but can be reduced to some simple geometric ideas involving the pullbacks and pushforwards of conormal distributions with respect to special maps, called $b$-fibrations, between manifolds with corners. This is described carefully when $\mathbb{K}=\mathbb{C}$ in [9, Theorem 12.42], and when $\mathbb{K}=\mathbb{R}$ in [18, Theorem 3.15]. The proofs in the other two cases follow exactly the same lines.

The other key facts we need concern the mapping properties of these operators. To state these we first describe the appropriate function spaces. Fix any smooth $\mathbb{K} \Theta$ metric $g$ on $M$. This determines the space $L^{2}\left(M ; d V_{g}\right)$, as well as the basic Hölder space $\Lambda_{\mathbb{K} \Theta}^{0, \alpha}(M)$, which by definition is the closure of bounded $\mathscr{C}^{\infty}$ functions with respect to the norm

$$
\|u\|_{0, \alpha}=\sup _{p \in M}|u(p)|+\sup _{\substack{p \neq q \\ \operatorname{dist}_{g}(p, q) \leq 1}} \frac{|u(p)-u(q)|}{\operatorname{dist}_{g}(p, q)^{\alpha}} .
$$


Next, for any $s \in \mathbb{N}$, set

$$
\begin{aligned}
& H_{\mathbb{K} \Theta}^{s}(M)=\left\{u: V_{1} \ldots V_{\ell} u \in L^{2}\left(M ; d V_{g}\right) \text { for all } V_{j} \in \mathscr{V}_{\mathbb{K} \Theta}, j \leq \ell, \text { and } \ell \leq s\right\}, \\
& \Lambda_{\mathbb{K} \Theta}^{s, \alpha}(M)=\left\{u: V_{1} \ldots V_{\ell} u \in \Lambda_{\mathbb{K} \Theta}^{0, \alpha} \text { for all } V_{j} \in \mathscr{V}_{\mathbb{K} \Theta}, j \leq \ell, \text { and } \ell \leq s\right\}
\end{aligned}
$$

and finally, for any defining function $x$ for $\partial M$ and $\delta \in \mathbb{R}$,

$$
x^{\delta} H_{\mathbb{K} \Theta}^{s}(M)=\left\{u=x^{\delta} v: v \in H_{\mathbb{K} \Theta}^{s}(M)\right\}, \quad x^{v} \Lambda_{\mathbb{K} \Theta}^{s, \alpha}(M)=\left\{u=x^{v} v: v \in \Lambda_{\mathbb{K} \Theta}^{s, \alpha}(M)\right\} .
$$

There is a somewhat loose relationship between certain of these weighted Sobolev and Hölder spaces. This is based on the fact that $x^{a} \in x^{\delta} H_{\mathbb{K} \Theta}^{s}$ (locally near $x=0$ ) if and only if $a>\delta+(n+d-1) / 2$; similarly, $x^{a} \in x^{\nu} \Lambda_{\mathbb{K} \Theta}^{s, \alpha}$ near $x=0$ if and only if $a \geq v$. Because of this we say that $x^{\delta} H_{\mathbb{K} \Theta}^{s}$ and $x^{v} \Lambda_{\mathbb{K} \Theta}^{s, \alpha}$ are commensurable when $v=\delta+(n+d-1) / 2$.

Proposition 10. Fix $\mu \in \mathbb{N}$ and $\sigma_{10}, \sigma_{01}, \delta \in \mathbb{R}$ such that $\sigma_{01}+\delta>0$ and $\sigma_{10}>\delta$. Let $A \in \Psi_{\mathbb{K} \Theta}^{-\mu, \sigma}(M)$. Then the maps

$$
\begin{gathered}
A: x^{\delta} H_{\mathbb{K} \Theta}^{k}(M) \rightarrow x^{\delta} H_{\mathbb{K} \Theta}^{k+\mu}(M), \\
A: x^{\delta+(n+d-1) / 2} \Lambda_{\mathbb{K} \Theta}^{k, \alpha}(M) \rightarrow x^{\delta+(n+d-1) / 2} \Lambda_{\mathbb{K} \Theta}^{k+\mu, \alpha}(M)
\end{gathered}
$$

are bounded.

We sketch a few points in the proof. First note that since these spaces are defined relative to $\mathbb{K} \Theta$ derivatives, and since $\mathscr{V}_{\mathbb{K} \Theta} \circ \Psi_{\mathbb{K} \Theta}^{j} \subset \Psi_{\mathbb{K} \Theta}^{j+1}$, we may immediately reduce to the case $\mu=0$. Furthermore, conjugating by $x^{-\delta}$ in the first case and $x^{-(\delta+(n+d-1) / 2)}$ in the second, and observing that $\tilde{x} / x$ lifts to a conormal function on $M_{\mathbb{K} \Theta}^{2}$ which is smooth (and nonvanishing) up to $B_{11}$, reduces us further to the unweighted case. Finally, the boundedness of $\Psi_{\mathbb{K} \Theta}^{0}(M)$ on $L^{2}\left(M ; d V_{g}\right)$ may be deduced via Hörmander's method of using the symbol calculus to find $B \in \Psi_{\mathbb{K} \Theta}^{0}$ satisfying $A^{*} A+B^{*} B=C^{2} \mathrm{Id}+R$ for some $C>0$ and $R \in \Psi_{\mathbb{K} \Theta}^{-\infty}$ and then using Cauchy-Schwarz to prove $L^{2}$ boundedness for operators of order $-\infty$. The boundedness of $\Psi_{\mathbb{K} \Theta}^{0}$ on $\Lambda_{\mathbb{K} \Theta}^{0, \alpha}$ is also deduced in two steps. Decompose $A$ into a sum $A^{\prime}+A^{\prime \prime}$ where the Schwartz kernel of $A^{\prime}$ vanishes to infinite order at $B_{10}$ and $B_{01}$ and that of $A^{\prime \prime}$ is smooth across the diagonal. The boundedness of $A^{\prime}$ on Hölder spaces is equivalent to the standard local boundedness of pseudodifferential operators (of order 0) on a neighbourhood in $\mathbb{R}^{n}$ (cf. [27]). This argument is discussed in detail in [18] for the case $\mathbb{K}=\mathbb{R}$.

Proposition 11. Suppose that $A \in \Psi_{\mathbb{K} \Theta}^{\mu, \sigma}(M)$, where $\sigma=\left(\sigma_{10}, \sigma_{01}, \sigma_{11}\right)$ indicates conormal order of vanishing at each of the three boundary faces $B_{10}, B_{01}, B_{11}$. If $\mu<0$ and $\sigma_{11}>0$, and if $\sigma_{01}+\delta>0$ and $\sigma_{10}>\delta$, then $A$ is compact on $x^{\delta} L^{2}$ and on $x^{\delta+(n+d-1) / 2} \Lambda_{\mathbb{K} \Theta}^{0, \alpha}$.

This follows directly from the Arzelà-Ascoli theorem. 


\section{The parametrix construction for fully elliptic operators}

We finally apply the theory of $\mathbb{K} \Theta$ operators outlined above to prove that under certain hypotheses, the linearized gauged Einstein operator $L^{g}$ is an isomorphism on certain weighted $L^{2}$ and Hölder spaces. We state the result in slightly greater generality for an arbitrary generalized Laplacian $P=\nabla^{*} \nabla+R \in \operatorname{Diff}_{\mathbb{K} \Theta}^{2}(M ; E)$, associated to a $\mathbb{K} \Theta$ metric $g$. The symmetry of this operator with respect to the volume form $d V_{g}$ simplifies some of the numerology below, but all of these results have direct analogues for more general fully elliptic $\mathbb{K} \Theta$ operators.

Definition 12. The generalized Laplacian $P^{g}$ is fully elliptic if its $\mathbb{K} \Theta$ symbol is invertible as a section of $\mathscr{C}^{\infty}\left(\mathbb{K} \Theta T^{*} \bar{M} ; \operatorname{Hom}(E)\right)$, and if, in addition, its normal operator $N\left(P^{g}\right)$, which is identified via Proposition 7 with the corresponding operator $P^{g_{0}}$ on the model hyperbolic space $\mathbb{K} H^{m}$, is invertible as an unbounded operator on $L^{2}\left(\mathbb{K} H^{m} ; E_{0}\right)$.

Before stating the main theorem of this subsection, let us explore the relationship of this full ellipticity condition with the indicial root structure of $P^{g}$. The indicial roots of $P^{g}$ are the roots of the indicial polynomial for $P^{g}$, and give the rates of vanishing of formal solutions of this operator. If $\zeta$ is an indicial root, then there exists some $\phi(y)$ such that $P\left(x^{\zeta} \phi(y)\right)=\mathscr{O}\left(x^{\zeta+1}\right)$. The indicial roots of $P^{g}$ and of its normal operator $N\left(P^{g}\right)$ are the same. The indicial roots are arranged symmetrically around $(n+d-1) / 2$ in $\mathbb{C}$. The complement in $\mathbb{R}$ of the set of real parts of all indicial roots of $P$ is a union of open intervals and half-lines, again symmetric around $(n+d-1) / 2$. The significance of these intervals is as follows. First, if $\delta+(n+d-1) / 2$ is the real part of some indicial root, hence at the boundary of two contiguous intervals, then neither of the mappings

$$
\begin{gathered}
P: x^{\delta} H_{\mathbb{K} \Theta}^{2}\left(M, E ; d V_{g}\right) \rightarrow x^{\delta} L^{2}\left(M, E ; d V_{g}\right), \\
P: x^{\delta+(n+d-1) / 2} \Lambda_{\mathbb{K} \Theta}^{2, \alpha}(M, E) \rightarrow x^{\delta+(n+d-1) / 2} \Lambda_{\mathbb{K} \Theta}^{0, \alpha}(M, E)
\end{gathered}
$$

has closed range. This is straightforward to check from basic definitions. Significantly deeper is

Theorem 13. Let $P^{g} \in \operatorname{Diff}_{\mathbb{K} \Theta}^{2}(M, E)$ be a fully elliptic generalized Laplacian. Define $\delta_{0}$ by the condition that $(n+d-1) / 2 \pm \delta_{0}$ are the real parts of the indicial roots of $P$ closest to $(n+d-1) / 2$. If $\delta_{0}>0$ and $|\delta|<\delta_{0}$, then (3.8) and (3.9) are both Fredholm, and are isomorphisms if and only if the nullspace of $P^{g}$ on $L^{2}\left(M ; d V_{g}\right)$ is trivial.

Proof. The fact that these mappings are Fredholm when $|\delta|<\delta_{0}$ will follow immediately if we can establish the existence of a parametrix $G \in \Psi_{\mathbb{K} \Theta}^{-2, \sigma, 0}(M ; E)$ for $P$ with the property that $P G-I=G P-I=Q \in \Psi_{\mathbb{K} \Theta}^{-\infty, \sigma, \infty}(M ; E)$. Here for convenience we set $\tau=(n+d-1) / 2+\delta_{0}$ and $\sigma=\left(\sigma_{10}, \sigma_{01}\right)=(\tau, \tau)$. The final index (here 0 or $\left.\infty\right)$ corresponds to $\sigma_{11}$.

This parametrix is constructed in stages. We first choose an element $G_{0}$ in the small calculus, i.e. $G_{0} \in \Psi_{\mathbb{K} \Theta}^{-2}(M ; E)$, so that $P G_{0}=I-Q_{0}, Q_{0} \in \Psi_{\mathbb{K} \Theta}^{-\infty}$. This uses only the symbol calculus and the symbol ellipticity of $P$, and proceeds exactly as in the usual (local) elliptic parametrix construction. 
For the second step we seek a correction term $G_{1} \in \Psi_{\mathbb{K} \Theta}^{-\infty, \sigma, 0}$ chosen so that the remainder term $Q_{1}=I-P\left(G_{0}+G_{1}\right)$ lies in $\Psi_{\mathbb{K} \Theta}^{-\infty, \sigma, 1}$, and in particular is compact. For this we must solve the normal problem $N(P) N\left(G_{1}\right)=N\left(Q_{0}\right) \in \mathscr{C}_{0}^{\infty}\left(\mathbb{K} H^{m} ; E\right)$. By the second part of the full ellipticity hypothesis there is a unique solution to this equation in $L^{2}\left(\mathbb{K} H^{m}, E\right)$ and (since the right hand side is $\mathscr{C}_{0}^{\infty}$ ) it is a simple matter to check that the solution is conormal at the boundaries of the quarter-sphere fibres of the front face. Indeed, using the analysis from [2, Proposition I.2.2] giving the decay of the Green function of $P$ on $\mathbb{K} H^{m}$, we see that $N\left(G_{0}\right) \in \mathscr{A}^{\sigma}\left(S_{++}^{n-1}\right)$, where $\sigma=(\tau, \tau)$ gives the orders of conormal vanishing at the two boundaries of the quarter-sphere fibres.

Using $|\delta|<\delta_{0}$, we see from Proposition 10 that $G_{1}$ and $Q_{1}$ are bounded between these weighted spaces; from Proposition 11 we also find that $Q_{1}$ is compact. This already shows that $P^{g}$ is Fredholm. However, it is useful to refine this parametrix further.

Using the composition formula for $\mathbb{K} \Theta$ pseudodifferential operators, we see that the iterated compositions of this error term with itself vanish to increasingly high order at the front face, specifically $Q_{1}^{j} \in \Psi_{\mathbb{K} \Theta}^{-\infty, \sigma, j}$. We can therefore take an asymptotic sum of the series $R \sim \sum_{j=1}^{\infty} Q_{1}^{j}$ as an element of $\Psi_{\mathbb{K} \Theta}^{-\infty, \sigma, 1}$. Now multiply $P\left(G_{0}+G_{1}\right)=I-Q_{1}$ on the right by $I+R$. We see that $G^{\prime}=\left(G_{0}+G_{1}\right)(I+R)$ satisfies $P G^{\prime}=I-Q^{\prime}$ where $Q^{\prime} \in \Psi_{\mathbb{K} \Theta}^{-\infty, \sigma, \infty}$.

This error term lies in the very residual space of smoothing operators with Schwartz kernels which are conormal on $M^{2}$. One consequence is that elements of the nullspace of $L$ in either of these function spaces are conormal and vanish like $x^{\tau}$. Furthermore, since these very residual operators form a semi-ideal (on one of these weighted $L^{2}$ spaces, say), a standard argument (cf. [18, proof of Theorem 6.1]) shows that the true generalized inverse $G$ of any of the maps between weighted spaces, which a priori is only defined as a bounded operator, is actually an element of $\Psi_{\mathbb{K} \Theta}^{-2, \sigma, 0}$; the error term $P G-I=$ $G P-I=Q$ is the projector onto the nullspace and is still very residual. If the nullspace is trivial, then $Q=0$ and hence $P$ is invertible.

We remark, but do not prove, that if $\delta$ lies in any of the other open intervals or half-lines described above, then these maps have closed range but are not Fredholm since either the kernel or cokernel is infinite-dimensional.

With not much more effort, we can prove that the Schwartz kernel of $G$ has a polyhomogeneous expansion at all boundary faces of $M_{\mathbb{K} \Theta}^{2}$ (as well as a polyhomogeneous expansion along the lifted diagonal of this space which is smoothly extendible across the front face). This implies that $G$ maps polyhomogeneous sections to polyhomogeneous sections, and also shows that any section $\kappa$ which satisfies $L \kappa=0$ (even just in a neighbourhood of infinity) must have a complete polyhomogeneous expansion there.

\section{The resolvent family}

The invertibility of the linearized gauged Einstein operator $L^{g}$ on weighted Hölder spaces, which is a direct consequence of Theorem 13 and Proposition 10, is the key ingredient in the deformation theory of $\mathrm{A} \mathbb{K H}$ Einstein metrics. For the analogous result on products of $\mathrm{A} \mathbb{K} \mathrm{H}$ spaces, we shall use a spectral synthesis formula for the inverse of this operator 
which expresses it in terms of the resolvent families of the corresponding operators on each factor. In preparation for this, we now recall the basic theory of these resolvent families in terms of the $\mathbb{K} \Theta$ calculus and prove some estimates on their Schwartz kernels which are uniform in the spectral parameter.

First let us recall some results from [2, §I.4]. Define $\delta_{0}=\delta_{0}^{\mathbb{K}}$ for the operator $L^{g}$ as in the statement of Theorem 13; then

$$
\begin{array}{ll}
\delta_{0}=(n+d-1) / 2 & \text { for } \mathbb{K}=\mathbb{R} \text { or } \mathbb{C}, \\
\delta_{0}>(n+d-1) / 2 & \text { for } \mathbb{K}=\mathbb{H} \text { or } \mathbb{O} .
\end{array}
$$

This means that the interval of weights $\delta$ for which $L^{g}$ is Fredholm is exactly $(0, n+d-1)$ in the real and complex cases, and is larger in the quaternionic and octonionic cases.

By definition, the resolvent of $L^{g}$ is the family of $L^{2}$ bounded operators $\left(L^{g}-\lambda\right)^{-1}$, which exists precisely when $\lambda \notin \operatorname{spec}\left(L^{g}\right)$. We wish to recognize these operators as elements of $\Psi_{\mathbb{K} \Theta}^{-2, *}(M)$, depending holomorphically on $\lambda$ in an appropriate sense. This will follow from Theorem 13, which in turn requires

Lemma 14. The operator $L^{g}-\lambda$ is fully elliptic (as a $\mathbb{K} \Theta$ operator) if and only if $\lambda \notin$ $\left[\delta_{0}^{2}, \infty\right)$.

Proof. First note that

$$
\mathbb{K} \Theta \sigma_{2}\left(L^{g}-\lambda\right)={ }^{\mathbb{K} \Theta} \sigma_{2}\left(\nabla^{*} \nabla\right)=|\zeta|^{2} \mathrm{Id},
$$

which is obviously invertible. In addition, $N\left(L^{g}-\lambda\right)=L^{g_{0}}-\lambda$, so we conclude that $L^{g}-\lambda$ is fully elliptic if and only if $\lambda \notin \operatorname{spec}\left(L^{g_{0}}\right)$.

The indicial operator of $L^{g_{0}}$ is a second order matrix-valued ordinary differential operator, and the indicial roots correspond to solutions of the form $x^{\zeta} \kappa_{0}$, where $\kappa_{0}$ is a constant symmetric 2-tensor. By reducing to the various irreducible components in $\mathrm{Sym}^{2}$, we obtain them as the roots of a finite number of quadratic polynomials $\zeta^{2}-(n+d-1) \zeta+\alpha$, where $\alpha$ is a constant depending on dimension and the irreducible component of the decomposition. The roots from any one of these polynomials are $(n+d-1) / 2 \pm \frac{1}{2} \sqrt{(n+d-1)^{2}-4 \alpha}$. For some $\alpha_{0}$ we obtain the roots with real part closest to $(n+d-1) / 2$, that is, $(n+d-1) / 2 \pm \delta_{0}$. So we see that each $\alpha \leq \alpha_{0}$. Now, the indicial roots of $L^{g_{0}}-\lambda$ are the roots of the various polynomials $\zeta^{2}-(n+\bar{d}-1) \zeta+\alpha+\lambda$, hence are equal to

$$
(n+d-1) / 2 \pm \frac{1}{2} \sqrt{(n+d-1)^{2}-4 \alpha-4 \lambda}
$$

Define

$$
\delta_{0}(\lambda)=\min _{\alpha} \frac{1}{2} \operatorname{Re} \sqrt{(n+d-1)^{2}-4 \alpha-4 \lambda}=\operatorname{Re} \sqrt{\delta_{0}^{2}-\lambda} .
$$

By Theorem 13, $L^{g_{0}}-\lambda$ is at least Fredholm on $L^{2}$ provided

$$
\delta_{0}(\lambda)>0
$$

or equivalently, if $\lambda \notin\left[\delta_{0}^{2}, \infty\right)$. 
So far we have proved that if $\lambda$ is outside this half-line, then $L^{g_{0}}-\lambda$ is at least Fredholm. This shows that $\operatorname{spec}\left(L^{g_{0}}\right)$ is the union of $\left[\delta_{0}^{2}, \infty\right)$ and finite point spectrum of multiplicity in the half-line $\left(-\infty, \delta_{0}^{2}\right)$. However, this point spectrum must be empty, since otherwise, if $L^{g_{0}} \phi=\widehat{\lambda} \phi$ for some $\widehat{\lambda}<\delta_{0}^{2}$ and $\phi \in L^{2}$, then the subspace spanned by all translates of $\phi$ by isometries of $\mathbb{K} H^{m}$ would be infinite-dimensional, contradicting the fact that $L^{g_{0}}-\widehat{\lambda}$ is Fredholm. This finishes the proof.

The same reasoning leads to

Theorem 15. Let $L^{g}$ be the linearized gauged Einstein operator on the manifold $M$ with $\mathbb{K} \Theta$ metric $g$. Then $L^{g}-\lambda$ is Fredholm if and only if $\lambda \notin\left[\delta_{0}^{2}, \infty\right)$. More precisely,

$$
\operatorname{spec}\left(L^{g}\right)=\left[\delta_{0}^{2}, \infty\right) \cup\left\{\lambda_{i}\right\}_{i=1}^{N},
$$

where $\lambda_{i}$ lies in $\left(-\infty, \delta_{0}^{2}\right)$ and is an $L^{2}$ eigenvalue of finite multiplicity.

An $\mathrm{A} \mathbb{K} H$ Einstein space $(M, g)$ is nondegenerate if and only if 0 is not in this point spectrum.

We shall need to know slightly more about the dependence of the inverse on $\lambda$.

Proposition 16. Fix $\epsilon>0$ and define $\Omega_{\epsilon} \subset \mathbb{C} \backslash\left[\delta_{0}^{2}, \infty\right)$ to consist of the set of all $\lambda$ for which $\delta_{0}(\lambda)>\epsilon$. Let $\tau_{\varepsilon}=(n+d-1) / 2+\varepsilon$ and $\sigma_{\varepsilon}=\left(\tau_{\varepsilon}, \tau_{\varepsilon}, 0\right)$. Then for each $\varepsilon>0$, the resolvent family

$$
\Omega_{\varepsilon} \ni \lambda \mapsto R(\lambda)=\left(L^{g}-\lambda\right)^{-1} \in \Psi_{\mathbb{K} \Theta}^{-2, \sigma_{\varepsilon}}\left(M ; \operatorname{Sym}^{2}\left({ }^{\mathbb{K} \Theta} T^{*} M\right)\right)
$$

is meromorphic in the sense that the Schwartz kernels of these operators, as elements of a fixed space of distributions, depend meromorphically on $\lambda$. The poles occur only at each $\lambda_{i}$ and are all simple; the residues are the finite rank orthogonal projections onto the corresponding eigenspaces.

The proof is based on the fact that the model resolvent $\left(L^{g_{0}}-\lambda\right)^{-1}$ is itself holomorphic, which can be checked by direct ODE analysis, and the analytic Fredholm theorem. This is the direct generalization of [20] and [9] (cf. also [12]).

We conclude this section by proving uniform estimates for the off-diagonal Schwartz kernel of this resolvent when $\lambda=i \mu$ lies on the imaginary axis.

Proposition 17. Let $(M, g)$ and $L^{g}$ be as above. When $\mu \in \mathbb{R}$, the indicial root of $L^{g}-i \mu$ which has the smallest strictly positive real part is equal to

$$
\zeta(\mu)=\frac{n+d-1}{2}+\sqrt{\delta_{0}^{2}-i \mu} .
$$

In particular $\gamma(\mu):=\operatorname{Re} \zeta(\mu) \geq n+d-1$ with equality if and only if $\mu=0$. Furthermore,

$$
\zeta(\mu) \sim \sqrt{|\mu| / 2}(1 \pm i) \quad \text { as } \mu \rightarrow \pm \infty
$$


Let $K\left(z, z^{\prime}, i \mu\right)$ be the Schwartz kernel of the resolvent of this operator. Then there exists $\mu_{0}>0$ such that for any $c>0$ and $\varepsilon \in(0,1)$, if $z, z^{\prime} \in M$ satisfy $d\left(z, z^{\prime}\right) \geq c>0$ and $|\mu| \geq \mu_{0}$, we have

$$
\left|K\left(z, z^{\prime}, i \mu\right)\right| \leq C \mu^{-1} x^{(1-\varepsilon) \gamma(\mu) d\left(z, z^{\prime}\right)}
$$

where the constant $C$ is independent of $\mu$. A similar estimate holds for all b-derivatives of $K$.

Proof. The calculation of the indicial root and the statements about its asymptotics are straightforward, based on the remarks in the proof of Lemma 14. As for the main assertion, first suppose that $f \in \mathscr{C}_{0}^{\infty}(M),|f| \leq 1$, and define $u=u_{\mu}=R(i \mu) f$. We claim that for any $\varepsilon>0$, there exists a constant $C_{\varepsilon}>0$ such that for $|\mu| \geq \mu_{0}$,

$$
|u| \leq C \mu^{-1} x^{(1-\varepsilon) \gamma(\mu)} .
$$

In particular, $C$ is independent of $\mu$ (and in addition, sup $|u|$ depends linearly on sup $|f|$ ).

To prove this, first recall that since $R(i \mu) \in \Psi_{\mathbb{K} \Theta}^{-2, \gamma(\mu), \gamma(\mu)}(M)$, it is immediate that $|u| \leq A_{\mu} x^{\gamma(\mu)}$; the issue is to prove the uniformity in $\mu$ of the constant $A$. Suppose this fails, i.e. suppose there exists a sequence of $\mu$ tending to infinity so that

$$
\sup _{z \in M} \mu|u(z)| x^{-(1-\varepsilon) \gamma(\mu)}=A_{\mu} \rightarrow \infty .
$$

This supremum is attained at a point $q_{\mu} \in M$, so if we define

$$
w(z)=\left(\mu x\left(q_{\mu}\right)^{-(1-\varepsilon) \gamma(\mu)} / A_{\mu}\right) u(z),
$$

then

$$
|w(z)| \leq\left(x / x\left(q_{\mu}\right)\right)^{(1-\varepsilon) \gamma(\mu)}
$$

with equality at $z=q_{\mu}$ and

$$
\left(\mu^{-1} L-i\right) w(z)=\left(x\left(q_{\mu}\right)^{-(1-\varepsilon) \gamma(\mu)} / A_{\mu}\right) f(z) .
$$

We shall consider various cases depending on whether or not $q_{\mu}$ remains in a compact subset of $M$.

Suppose first that $x\left(q_{\mu}\right) \geq c>0$. Then the right hand side of (3.12) tends to zero uniformly. Let $B$ be a geodesic ball of radius 1 centred at $q_{\mu}$, fix a trivialization of the bundle over $B$ and suppose that $z$ are Riemann normal coordinates in this ball. Set $\xi=$ $\sqrt{\mu} z$, so that $\xi$ lies in a ball of radius $\sqrt{\mu}$ in $\mathbb{R}^{n+1}$. In terms of these coordinates,

$$
\mu^{-1} L=-\sum_{j=1}^{n+1} \frac{\partial^{2}}{\partial \xi_{j}^{2}}+\mathscr{O}\left(\mu^{-1 / 2}\right)
$$

The remainder term is a second order operator with coefficients which converge to 0 uniformly on compact sets in these expanding balls in $\mathbb{R}^{n+1}$. Using standard local elliptic 
theory, we can take a limit in (3.12), and obtain a function $w_{\infty}$ defined on the entire Euclidean space such that

$$
\left(\Delta_{\xi}-i\right) w_{\infty}=0, \quad\left|w_{\infty}\right| \leq 1, \quad\left|w_{\infty}(0)\right|=1 .
$$

However, no such function exists. To see this, take Fourier transform (for $w$ as an element of $\mathscr{S}^{\prime}\left(\mathbb{R}^{n+1}\right)$ ) and use that the full symbol $|\xi|^{2}-i$ is nowhere vanishing. Hence this case cannot occur.

Now suppose that $x\left(q_{\mu}\right) \rightarrow 0$. Pass to a subsequence so that $q_{\mu} \rightarrow \bar{q} \in \partial M$, and then apply a sequence of parabolic dilations $D_{\mu}$ based at appropriate points converging to $\bar{q}$ and with strength $x\left(q_{\mu}\right)^{-1}$ so that $D_{\mu}\left(q_{\mu}\right)=(1,0)$ in a fixed coordinate system $\tilde{z}$. We now proceed much as before. Let $B$ be a unit geodesic ball centred at $(1,0)$, and define $\xi=\sqrt{\mu} \tilde{z}$. The sequence of operators $\mu^{-1} D_{\mu}^{*} L$ converges to $\Delta_{\xi}$ as before. The bound on $\tilde{w}=D_{\mu}^{*} w$ now takes the form

$$
|\tilde{w}| \leq e^{(1-\varepsilon) \gamma(\mu) / \sqrt{\mu}}
$$

with equality at the origin; here $t=\log \left(x / x\left(q_{\mu}\right)\right)$, and we can assume this is the first coordinate $\xi_{1}$ in the $\xi$ system. We again pass to the limit. The limiting function $\tilde{w}_{\infty}$ satisfies $\left|\tilde{w}_{\infty}(0)\right|=1$,

$$
\left(\Delta_{\xi}-i\right) \tilde{w}_{\infty}=0, \quad\left|\tilde{w}_{\infty}\right| \leq e^{(1-\varepsilon) t / \sqrt{2}} .
$$

To analyze whether this is possible, note that this exponential bound on $\tilde{w}_{\infty}$ implies that its Fourier transform is well defined as an element of $\mathscr{S}^{\prime}$ on the subspace $\left\{\xi \in \mathbb{C}^{n+1}\right.$ : $\left.\operatorname{Im} \xi_{1}=(1-\varepsilon) / \sqrt{2}, \xi_{j} \in \mathbb{R}, j>1\right\}$. The symbol $\xi \cdot \xi-i$ is again invertible here, which precludes the existence of this limit; hence this case is also impossible. This proves that the function $u=R(i \mu) f$ satisfies the stated bound uniformly in $\mu$.

An essentially identical argument proves that a similar bound holds regardless of the location of the support of $f$. In other words, suppose that $\operatorname{supp}(f) \subset B_{1}\left(p_{\mu}\right)$ and $\sup |f| \leq 1$. Then for the $L^{2}$ solution to $(L-i \mu) u=f$, we have $|u(q)| \leq$ $C \mu^{-1} \exp \left(-(1-\varepsilon) \gamma(\mu) d\left(q, p_{\mu}\right)\right)$. The only modification needed is that if there is a sequence $u_{\mu}$ for which the constant increases without bound, and if the centre $q_{\mu}$ of the support of $f_{\mu}$ tends to infinity, then we parabolically rescale so as to obtain a sequence of problems $\left(L_{\mu}-i \mu\right) \tilde{u}_{\mu}=\tilde{f}_{\mu}$, where the rescaled operators $L_{\mu}$ converge to the limiting model operator for the $\mathbb{K} \Theta$ structure. The validity of the bound in this case follows by what we have done above.

We have now proved that $K\left(z, z^{\prime}, i \mu\right)$ decays like $e^{-(1-\varepsilon) \gamma(\mu) d\left(z, z^{\prime}\right)} \mu^{-1}$ for $d\left(z, z^{\prime}\right) \geq$ $c>0$ in a weak sense. More precisely, let $\mathscr{U}$ be any neighbourhood in $M \times M$ with compact closure which does not intersect the diagonal; then if $\sigma>2 n+2$, the $H^{-\sigma}$ norm of the restriction of the Schwartz kernel to $\mathscr{U}$ satisfies this bound. Using that $\left(L_{z}-i \mu\right) K=\left(L_{z^{\prime}}-i \mu\right) K=0$ away from the diagonal, we can estimate any $\mathscr{C}^{k}$ norm of $K$ in $\mathscr{U}$ at the cost of introducing an extra factor $\mu^{k+\sigma}$ into the estimate. This in turn may be absorbed into the exponential by decreasing the factor $\varepsilon$ slightly. This completes the proof of the $\mathscr{C}^{0}$ bound, and indeed also of bounds with respect to any $\mathscr{C}^{k}$ norm 
in the interior. In fact, it gives slightly more, namely that this bound holds even after applying any sequence of $\mathbb{K} \Theta$ vector fields to $K$ on the left and right; this is because $\mathbb{K} \Theta$ derivatives are controlled by powers of $L$, which as above are equivalent to powers of $\mu$.

To finish, we also need to check the conormal bounds, i.e. that the same estimates remain true if we apply any sequence of $b$ vector fields to $K$ on the left and right. For this we point out the following facts: first, since $K\left(z^{\prime}, z, i \mu\right)=K\left(z, z^{\prime},-i \mu\right)^{*}$, we need only the case where all $b$-derivatives are applied to the left $(z)$ factor; next, $K$ itself is conormal, so these $b$-derivatives behave well locally uniformly in $\mu$, i.e. it is only the large $\mu$ behaviour that might be problematic; finally, we can repeat the same proof as for $K$ itself, using at the final step to convert the weak bounds to strong ones that if $V$ is any $b$-vector field, then $[L, V]$ is a $\mathbb{K} \Theta$ operator of order 2 , hence is bounded by multiplication by $\mu$.

Remark 18. We have stated the results on the resolvent family for the linearized Einstein operator, but the results remain true for any geometric Laplacian, provided we choose $\delta_{0}$ as in Theorem 13. For example, on an asymptotically quaternionic hyperbolic space, Theorem 15 gives the spectrum of the Hodge Laplacian acting on differential forms (except when the degree equals half the dimension, then there is a zero eigenvalue of infinite multiplicity).

\section{Einstein deformation theory}

We now present some basic facts about the (Bianchi gauged) Einstein operator and its linearization. Using results from the last section, we review how this yields the deformation theory for rank one hyperbolic spaces in the class of $\mathrm{A} \mathbb{K} H$ Einstein spaces. This is contained in [2] for all $\mathbb{K}$ (see also [11] and [15] for the result when $\mathbb{K}=\mathbb{R}$ ), so the only novelty here is showing how this follows immediately through the use of the $\mathbb{K} \Theta$ calculus. These same arguments are used again in $\$ 6$ for a coupled generalization of these same equations, and in the product case in $\S 8$.

\subsection{The Einstein equation and the Bianchi gauge}

The Einstein equation $\mathrm{Ric}^{g}+\lambda g=0$ is not elliptic because of its diffeomorphism invariance. Amongst many viable gauge choices, the Bianchi gauge introduced in [2] is particularly convenient. Define the map from symmetric 2-tensors to 1 -forms, relative to the fixed background metric $g$,

$$
k \mapsto B^{g}(k)=\delta^{g} k+\frac{1}{2} d \operatorname{Tr}^{g} k .
$$

Note that $B^{g}(g)=0$, so the subspace of metrics $\tilde{g}$ close to $g$ which are in Bianchi gauge (i.e. $\left.B^{g}(\tilde{g})=0\right)$ is identified with the set of tensors $k$ near 0 such that $B^{g}(k)=0$. The system

$$
\operatorname{Ric}^{\tilde{g}}+\lambda \tilde{g}=0, \quad B^{g}(\tilde{g})=0,
$$


which is elliptic in the sense of Agmon-Douglis-Nirenberg, can be rolled up into the single elliptic equation

$$
N^{g}(k):=\operatorname{Ric}^{g+k}+\lambda(g+k)+\left(\delta^{g+k}\right)^{*} B^{g}(k)=0 .
$$

As proved in [2, Chapter 1], we have

Proposition 19. Suppose that $N^{g}(k)=0$, and in addition $\left|B^{g}(k)\right|$ tends to 0 at the boundary and the Ricci curvature of $g+k$ is nonpositive and strictly negative somewhere. Then $g+k$ satisfies (4.2), i.e. is Einstein and in Bianchi gauge.

The proof follows from the Weitzenböck formula

$$
B^{g+k} N^{g}(k)=\delta^{g+k}\left(\delta^{g+k}\right)^{*} B^{g}(k)=\left(\left(\nabla^{g+k}\right)^{*} \nabla^{g+k}-\operatorname{Ric}^{g+k}\right) B^{g}(k)
$$

and the Bochner technique.

From the same Weitzenböck formula, the converse follows almost immediately, that is, any Einstein metric $\tilde{g}$ close to $g$ can be put in the Bianchi gauge to satisfy the system (4.2). More precisely, let Diff denote the set of all diffeomorphisms on the $\mathrm{A} \mathbb{K} H$ space $M$ which are close to the identity and exponentials of vector fields $X \in x^{\nu} \Lambda_{\mathbb{K} \Theta}^{3, \alpha}(M), \mathscr{M}$ the set of all metrics $\tilde{g}=g+k$ with $k \in x^{\nu} \Lambda_{\mathbb{K} \Theta}^{2, \alpha}(M)$, and $\mathscr{S} \subset \mathscr{M}$ the set of metrics $\tilde{g}$ which satisfy $B^{g}(\tilde{g})=0$. Then one has the following slice statement [2, Chapter 1]:

Proposition 20. If $\mathrm{Ric}^{g}<0$, then the natural map

$$
\operatorname{Diff} \times \mathscr{S} \rightarrow \mathscr{M}, \quad(\tilde{g}, \Phi) \mapsto \Phi^{*}(\tilde{g}),
$$

is a local homeomorphism.

An advantage of this gauge is that the linearization takes the simple form

$$
L^{g} \kappa:=\left.2 D N^{g}\right|_{0}(\kappa)=\nabla^{*} \nabla \kappa-2 \stackrel{R}{\kappa}+\operatorname{Ric} \circ \kappa+\kappa \circ \operatorname{Ric}+2 \lambda \kappa
$$

here

$$
(\stackrel{\circ}{R} \kappa)_{i j}=R_{i p j q} \kappa^{p q}, \quad(\operatorname{Ric} \circ \kappa)_{i j}=\operatorname{Ric}_{i}^{p} \kappa_{p j}, \quad(\kappa \circ \mathrm{Ric})_{i j}=\kappa_{i}^{p} \operatorname{Ric}_{p j}
$$

and all curvatures and covariant derivatives are computed relative to $g$. Note in particular that if $\operatorname{Ric}^{g}=-\lambda g$, then

$$
L^{g}=\nabla^{*} \nabla-2 \stackrel{\circ}{R}
$$




\subsection{Deformation theory for $A \mathbb{K} H$ Einstein spaces}

We now review the basic deformation theory for $\mathrm{A} \mathbb{K} H$ Einstein spaces, proved originally in [11] when $\mathbb{K}=\mathbb{R}$, and in [2] in the other two cases. We do not discuss the more subtle aspects of this deformation theory, but restrict attention to the simpler case of perturbations of nondegenerate $\mathrm{A} \mathbb{K} H$ Einstein metrics.

Definition 21. An AKKH Einstein metric $g$ is said to be nondegenerate if the $L^{2}$ nullspace of the linearized Bianchi-gauged Einstein operator $L^{g}$ is trivial.

Recall that $g$ is nondegenerate if for any $v>-\delta_{0}^{\mathbb{K}}$, the nullspace of $L^{g}$ on $x^{\nu+(n+d-1) / 2} \Lambda_{\mathbb{K} \Theta}^{2, \alpha}\left(M, \operatorname{Sym}^{2}\left(T^{*} M\right)\right)$ is trivial. This follows from the regularity theorem stating that if $L^{g} \kappa=0$ and $|\kappa| \leq C x^{\nu^{\prime}}$ for some $v^{\prime}>(n+d-1) / 2-\delta_{0}$, then $\kappa \in \mathscr{A}_{\text {phg }}^{(n+d-1) / 2+\delta_{0}}$, and in particular $\kappa \in L^{2}$.

The significance of this nondegeneracy condition is contained in

Proposition 22 ([11], [2], [15]). Let $g$ be a nondegenerate AKKH Einstein metric with $\mathscr{C}^{\infty}$ conformal infinity $\mathfrak{c}$. Then every $\mathscr{C}^{\infty}$ conformal infinity datum $\mathfrak{c}^{\prime}$ sufficiently close to $\mathfrak{c}$ in the $\mathscr{C}^{2, \alpha}$ topology is the conformal infinity of an $A \mathbb{K} H$ Einstein metric $g^{\prime}$ such that $g^{\prime}-g_{\mathfrak{c}^{\prime}} \in x^{\nu} \Lambda_{\mathbb{K} \Theta}^{2, \alpha}$ for some $v>0$. (Here $g_{\mathfrak{c}^{\prime}}$ is an $A \mathbb{K} H$ metric with conformal infinity $\mathfrak{c}^{\prime}$ constructed below in the proof.) This metric $g^{\prime}$ is unique amongst $A \mathbb{K} H$ metrics with the specified conformal infinity and such that $\left\|g^{\prime}-g_{\mathfrak{c}^{\prime}}\right\|_{2, \alpha, v}$ is small.

Proof. First define an extension operator which associates to the conformal infinity $\mathfrak{c}^{\prime}$ an AKH metric $g_{\mathfrak{c}^{\prime}}$. If $\mathfrak{c}=([\gamma], \eta)$, then we choose a product decomposition $(0, \epsilon) \times X$ of a collar neighbourhood of $\partial M$ and a radial coordinate $x$ so that $g$ has the form $\left(d x^{2}+\gamma\right) / x^{2}+\eta^{2} / x^{4}+k$ with $k \in x^{\nu} \Lambda_{\mathbb{K} \Theta}^{2, \alpha}$. (We can take the weight $v$ to equal 1 when $\mathfrak{c}$ is smooth, but could also use any smaller positive value.) Fixing a cutoff function $\chi(x)$ which equals 1 for $x \leq \epsilon / 3$ and vanishes for $x \geq 2 \epsilon / 3$, for any conformal infinity $\mathfrak{c}^{\prime}=\left(\left[\gamma^{\prime}\right], \eta^{\prime}\right)$, set

$$
g_{\mathfrak{c}^{\prime}}=(1-\chi(x)) g+\chi(x)\left(\frac{d x^{2}+\gamma^{\prime} / 4}{x^{2}}+\frac{\left(\eta^{\prime}\right)^{2}}{4 x^{4}}+k\right) .
$$

(In the quaternionic case, the function spaces $x^{\nu} \Lambda_{\mathbb{K} \Theta}^{2, \alpha}$ vary with $\mathfrak{c}^{\prime}$ so one has also to choose $k$ varying continuously with $\mathfrak{c}^{\prime}$.) Write $f_{\mathfrak{c}^{\prime}}=N^{g_{\mathfrak{c}^{\prime}}}(0)=\operatorname{Ric}^{g_{\mathfrak{c}^{\prime}}}+\lambda g_{\mathfrak{c}^{\prime}}$; then from the form of the metric $g_{\mathfrak{c}^{\prime}}$ (it is asymptotically hyperbolic in the sense of Definition 2) it is clear that it is an asymptotic solution of the Einstein equation: $f_{\mathfrak{c}^{\prime}}=O\left(x^{\nu}\right)$, and more precisely

$$
\left\|f_{\mathfrak{c}^{\prime}}\right\|_{0, \alpha, \nu} \leq C\left(\left\|\gamma^{\prime}-\gamma\right\|_{2, \alpha}+\left\|\eta^{\prime}-\eta\right\|_{2, \alpha}\right)
$$

The second step is then to deform $g_{\mathfrak{c}^{\prime}}$ into an exact solution $g_{\mathfrak{c}^{\prime}}+k$ of the Einstein equation. Taylor expansion gives

$$
N^{g_{\mathfrak{c}^{\prime}}}(k)=f_{\mathfrak{c}^{\prime}}+L^{\mathfrak{c}^{\prime}} k+Q\left(\mathfrak{c}^{\prime}, k\right),
$$

where the second term on the right is the linearized Bianchi-gauged Einstein operator at $g_{\mathfrak{c}^{\prime}}$. The nondegeneracy of $L^{g}$ implies that $L^{\mathfrak{c}^{\prime}}$ is also invertible if $\mathfrak{c}^{\prime}$ is close enough 
to $\mathfrak{c}$; we denote its inverse by $G^{\mathfrak{c}^{\prime}}$. When $\mathfrak{c}^{\prime}$ is $\mathscr{C}^{\infty}$, this operator is a $\mathbb{K} \Theta$ pseudodifferential operator of order -2 , and for $0<v \leq 1$,

$$
G^{\mathfrak{c}^{\prime}}: x^{\mathcal{v}} \Lambda_{\mathbb{K} \Theta}^{0, \alpha}\left(M, \operatorname{Sym}^{2}\left(T^{*} M\right)\right) \rightarrow x^{\mathcal{v}} \Lambda_{\mathbb{K} \Theta}^{2, \alpha}\left(M, \operatorname{Sym}^{2}\left(T^{*} M\right)\right)
$$

is bounded. Furthermore, the norm of this operator is bounded independently of $\mathfrak{c}^{\prime}$ in a neighbourhood of $c$. When $\mathbb{K}=\mathbb{H}$, the function spaces vary with $\eta^{\prime}$ (i.e. with the distribution $\mathscr{D}^{\prime}$ ).

Now write the equation to be solved as

$$
k=-G^{\mathfrak{c}^{\prime}}\left(f_{\mathfrak{c}^{\prime}}+Q\left(\mathfrak{c}^{\prime}, k\right)\right) .
$$

This makes sense since for our initial approximated solution $g_{\mathfrak{c}^{\prime}}$ the error $f_{\mathfrak{c}^{\prime}}$ is in the domain of $G^{\mathfrak{c}^{\prime}}$. Now the right side defines a contraction mapping when $\mathfrak{c}^{\prime}$ is sufficiently close to $\mathfrak{c}$, and from this we immediately obtain a unique solution $k$. The metric $g_{\mathfrak{c}^{\prime}}+k$ is an $\mathrm{A} \mathbb{K} H$ metric which solves the gauged Einstein equation. By Proposition 19, it is in fact an $\mathrm{A} \mathbb{K} H$ Einstein metric in Bianchi gauge.

Remark 23. A precise statement about the regularity of $g$ and $g^{\prime}$ near $\partial X$ has been omitted, and indeed this is a subtle issue. There is a substantial difference between understanding the dependence of the asymptotic regularity for an arbitrary AIKH Einstein metric on that of its conformal infinity data, and the same question for such a metric obtained by perturbation from one which is a priori known to be polyhomogeneous. The reason is that in the former case one needs to deal explicitly with the gauge conditions, while in the latter, the gauge choice is part of the setup and the perturbation term automatically satisfies a $\mathbb{K} \Theta$ elliptic equation. Here is a summary of what is known.

For the nonperturbative case, when $\mathbb{K}=\mathbb{R}$ and the conformal infinity data $\mathfrak{c}$ of the $\mathrm{A} \mathbb{R H}$ Einstein metric $g$ is $\mathscr{C}^{\infty}$, then $g$ is polyhomogeneous, and in fact, in even dimensions has a smooth conformal compactification [5], [13]. The corresponding result has not been proved in the other cases (except when $\mathbb{K}=\mathbb{C}$ and $g$ is Kähler-Einstein [16]), but is surely true by essentially the same method as in [5].

As for the perturbative case, a simple adaptation of the argument in [19], which depends only on the commutation properties of $\Psi_{\mathbb{K} \Theta}^{*}$ with $b$-vector fields on $X$, proves that if the perturbed conformal infinity data $\mathfrak{c}^{\prime}$ is $\mathscr{C}^{\infty}$, then the solution $k$, and hence the metric $g^{\prime}$, constructed above is polyhomogeneous.

These issues will not be emphasized here, and we shall tacitly assume the polyhomogeneous regularity of A $\mathbb{K H}$ metrics with $\mathscr{C}^{\infty}$ conformal infinities. To ease the reader's conscience, however, since we will not supply the full proof of that fact, note that this issue only arises in $\S 7$, and one can easily adapt the arguments to accommodate metrics with lower regularity, as we discuss briefly there.

\section{Asymptotically product hyperbolic metrics and their conformal infinities}

Let $M_{i}^{n_{i}+1}=G_{i} / K_{i}$ be an $\mathrm{A} \mathbb{K}_{i} \mathrm{H}$ space, $i=1,2$. The boundary at infinity, $S^{n_{i}}=$ $K_{i} / H_{i}$, is equipped with the standard $\mathbb{K}_{i}$-contact distribution $\mathscr{D}_{i}$, which has a conformal 
$H_{i}$ structure inducing a compatible conformal class $\left[\gamma_{i}\right]$. The product hyperbolic space $M=M_{1} \times M_{2}$ is a (reducible) rank two symmetric space with Furstenberg boundary $X=S^{n_{1}} \times S^{n_{2}}$. Let $g_{i}$ be the standard metric on each factor, so that Ric ${ }^{g_{i}}+\lambda_{i} g_{i}=0$. Then

$$
g=\lambda_{1} g_{1}+\lambda_{2} g_{2}
$$

is Einstein with

$$
\operatorname{Ric}^{g}+g=0 .
$$

We begin this section by describing a class of boundary structures on $X$, called $\left(G_{1} \times G_{2}\right)$-conformal structures, which constitute the conformal infinity data for the class of Einstein metrics we eventually construct. The problem of extending one of these boundary structures to a metric on $M$ which is asymptotically Einstein in an appropriately strong sense is far from immediate. The 'obvious' extension has Einstein tensor vanishing in some sector near $X$, but not uniformly near infinity. The main goal of this section is to explore the geometry of asymptotically product hyperbolic metrics in order to find the correct compatibility conditions for metrics which are asymptotically Einstein in this stronger sense. Their construction is carried out in the next section.

\section{1. $\left(G_{1} \times G_{2}\right)$-conformal structures}

Using the notation above, we make

Definition 24. A $\left(G_{1} \times G_{2}\right)$-conformal structure on $X$ consists of a pair of distributions, each equipped with a conformal class of metrics, $\left(\mathscr{D}_{i},\left[\gamma_{i}\right]\right), i=1,2$, such that

(1) the distributions $\mathscr{F}_{i}=\mathscr{D}_{i}+\left[\mathscr{D}_{i}, \mathscr{D}_{i}\right]$ are integrable;

(2) $\mathscr{F}_{1} \oplus \mathscr{F}_{2}=T X$;

(3) the pair $\left(\mathscr{D}_{i}, \mathscr{F}_{i} / \mathscr{D}_{i}\right)$ with induced bracket

$$
[,]: \mathscr{D}_{i} \times \mathscr{D}_{i} \rightarrow \mathscr{F}_{i} / \mathscr{D}_{i}
$$

is isomorphic to the graded $\mathbb{K}_{i}$-Heisenberg algebra;

(4) $\mathscr{D}_{i}$ is equipped with a conformal $H_{i}$ structure, compatible with the bracket, and inducing the conformal metric $\left[\gamma_{i}\right]$;

(5) $\left[\mathscr{D}_{1}, \mathscr{D}_{2}\right] \subseteq \mathscr{D}_{1}+\mathscr{D}_{2}$.

The basic example, of course, is a product structure on $X=X_{1} \times X_{2}$ : here $\mathscr{D}_{1} \subset$ $\mathscr{F}_{1}=T X_{1} \oplus\{0\}$ and $\mathscr{D}_{2} \subset \mathscr{F}_{2}=\{0\} \oplus T X_{2}$, and each $\left(\mathscr{D}_{i},\left[\gamma_{i}\right]\right)$ is the pullback of a $G_{i}{ }^{-}$ conformal structure from $X_{i}$. Our main focus in this paper is with perturbations of these product structures. As we now indicate, there is substantial rigidity in the deformation theory, and nearby structures retain many vestiges of the product case.

Lemma 25. For $i=1,2$, let $X_{i}$ be a compact simply connected manifold with $G_{i}$ conformal structure $\left(\mathscr{D}_{i},\left[\gamma_{i}\right]\right)$. Then any small deformation of the product $\left(G_{1} \times G_{2}\right)$ conformal structure on $X=X_{1} \times X_{2}$ has the following properties:

1. the pairs of distributions $\left(\mathscr{D}_{1}, \mathscr{D}_{2}\right)$ and $\left(\mathscr{F}_{1}, \mathscr{F}_{2}\right)$ remain of product type; 
2. if $\mathbb{K}_{i}=\mathbb{R}$ or $\mathbb{C}$, then for that $i, \mathscr{D}_{i}$ remains fixed (up to a global diffeomorphism), but the deformation of $\left[\gamma_{i}\right]$ may depend on both factors in $X_{1} \times X_{2}$;

3. if $\mathbb{K}_{i}=\mathbb{H}$, then the distribution $\mathscr{D}_{i}$ varies amongst quaternionic contact structures, but since the conformal class $\left[\gamma_{i}\right]$ is determined by $\mathscr{D}_{i}$, any deformation of $\left[\gamma_{i}\right]$ depends only on the factor $X_{i}$;

4. if $\mathbb{K}_{i}=\mathbb{O}$, then both $\mathscr{D}_{i}$ and $\left[\gamma_{i}\right]$ remain fixed in the deformation (and in fact $X_{i}=S^{n_{i}}$ and the structure is standard).

In particular, the distribution $\mathscr{D}_{i}$ can change (modulo diffeomorphisms) only in the quaternionic case, and the conformal metric $\left[\gamma_{i}\right]$ may depend on both factors $X_{1}$ and $X_{2}$ only if $X_{i}$ is real or complex.

Proof. At the initial product structure, the leaves of the foliation corresponding to $\mathscr{F}_{i}$ are just the parallel copies of $X_{i}$. After a small deformation, the leaves are covering spaces for $X_{i}$, and since both $X_{1}$ and $X_{2}$ are simply connected, these leaves must remain diffeomorphic to $X_{i}$. In particular, the perturbed distributions $\mathscr{F}_{i}$ are still equal to the tangent bundles of the respective factors.

Now observe that as an immediate consequence of conditions 3 and 5,

$$
\left[\mathscr{F}_{1}, \mathscr{D}_{2}\right]=\left[\mathscr{D}_{1}+\left[\mathscr{D}_{1}, \mathscr{D}_{1}\right], \mathscr{D}_{2}\right] \subseteq \mathscr{F}_{1}+\mathscr{D}_{2}
$$

(by the Jacobi identity), and hence $\mathscr{D}_{2}$ is invariant along the leaves of the foliation corresponding to $\mathscr{F}_{1}$; similarly $\mathscr{D}_{1}$ is invariant along the leaves of the foliation for $\mathscr{F}_{2}$.

When $\mathbb{K}_{i}=\mathbb{R}, \mathscr{D}_{i}$ remains equal to the tangent bundle $T X_{i}$, while if $\mathbb{K}_{i}=\mathbb{C}$, then by Darboux's lemma, we may still assume that $\mathscr{D}_{i}$ remains fixed in the deformation.

In other words, in this deformation theory, we may as well assume that the distributions $\mathscr{D}_{i}$ and $\mathscr{F}_{i}$ remain of product type. The remaining assertions follow directly from this.

When $X_{1}$ or $X_{2}$ are not simply connected, we shall impose this product structure as a separate hypothesis:

Definition 26. A deformation of a product $\left(G_{1} \times G_{2}\right)$-conformal structure on $X=$ $X_{1} \times X_{2}$ is called globally integrable if (modulo diffeomorphism) the foliations $\mathscr{F}_{1}$ and $\mathscr{F}_{2}$ remain the tangent spaces of the two factors of $X=X_{1} \times X_{2}$.

It is possible to define $\left(G_{1} \times G_{2}\right)$-conformal structures on any closed manifold $X$ of the appropriate dimension. Looking ahead to the main goals of this paper, one could then try to extend this to an asymptotically Einstein metric on some manifold $M$ with two boundary hypersurfaces $F_{1}$ and $F_{2}$ and $X$ as its corner of codimension 2. However, we have already noted that this extension problem is not at all easy; in fact, the main difficulty seems to be the extension from $X$ to the boundary faces $F_{i}$. For this, it appears to be almost necessary that $X$ and $M$ be products, and that the metrics and boundary structures are globally rather similar to the ones considered here. Thus, in all that follows, we shall assume for simplicity that $X, M$ and the distributions $\mathscr{D}_{i}$ and $\mathscr{F}_{i}$ are products. 


\subsection{Asymptotically product hyperbolic metrics}

It is always possible to construct a complete metric on the interior of $M$ which is 'weakly' product hyperbolic and with any given $\left(G_{1} \times G_{2}\right)$-conformal structure on $X$ as its prescribed conformal infinity. In fact, we can just write down a formula which directly generalizes (2.4): let $x_{i}$ be defining functions for the boundary hypersurfaces $X_{i} \subset M_{i}$, and choose $\operatorname{Im} \mathbb{K}_{i}$-valued 1-forms $\eta_{i}$ defining $\mathscr{D}_{i}$ and compatible metrics $\gamma_{i}$ representing the given conformal classes. In a neighbourhood of $X$ of the form $(0, \epsilon)_{x_{1}} \times(0, \epsilon)_{x_{2}} \times X$, set

$$
g_{\gamma_{1}, \eta_{1}, \gamma_{2}, \eta_{2}}=\lambda_{1}\left(\frac{d x_{1}^{2}}{x_{1}^{2}}+\frac{\gamma_{1}}{4 x_{1}^{2}}+\frac{\eta_{1}^{2}}{4 x_{1}^{4}}\right)+\lambda_{2}\left(\frac{d x_{2}^{2}}{x_{2}^{2}}+\frac{\gamma_{2}}{4 x_{2}^{2}}+\frac{\eta_{2}^{2}}{4 x_{2}^{4}}\right) .
$$

Slightly more generally:

Definition 27. A metric $g$ on $M$ is weakly asymptotically product hyperbolic if

$$
g=g_{\gamma_{1}, \eta_{1}, \gamma_{2}, \eta_{2}}+k, \quad k \in\left(x_{1}+x_{2}\right)^{\nu} \Lambda^{2, \alpha}
$$

for some $v>0$. (The norms and covariant derivatives are with respect to the metric (5.1).) Then we say that $\left(\mathscr{D}_{1}, \mathscr{D}_{2},\left[\gamma_{1}\right],\left[\gamma_{2}\right]\right)$ is the conformal infinity of $g$.

Just as in the rank one setting, $g$ determines its conformal infinity. Conversely, replacing $\gamma_{i}$ and $\eta_{i}$ in (5.2) by any other conformal representatives

$$
\tilde{\gamma}_{i}=f_{i} \gamma_{i}, \quad \tilde{\eta}_{i}=f_{i} \eta_{i},
$$

where the $f_{i}$ are strictly positive smooth functions on $X$, yields a new metric

$$
\tilde{g}=\lambda_{1}\left(\frac{d x_{1}^{2}}{x_{1}^{2}}+\frac{f_{1} \gamma_{1}}{4 x_{1}^{2}}+\frac{f_{1}^{2} \eta_{1}^{2}}{4 x_{1}^{4}}\right)+\lambda_{2}\left(\frac{d x_{2}^{2}}{x_{2}^{2}}+\frac{f_{2} \gamma_{2}}{4 x_{2}^{2}}+\frac{f_{2}^{2} \eta_{2}^{2}}{4 x_{2}^{4}}\right) .
$$

We claim that up to a diffeomorphism $\Phi, \tilde{g}$ is asymptotically equivalent to $g$. Indeed, if $\Phi^{*} \tilde{x}_{i}=x_{i} / \sqrt{f_{i}}$ and $\left.\Phi\right|_{X}=\mathrm{id}$, then

$$
\frac{d \tilde{x}_{i}}{\tilde{x}_{i}}=\frac{d x_{i}}{x_{i}}-\frac{d f_{i}}{2 f_{i}} \quad \text { and } \quad\left|\frac{d \tilde{x}_{i}}{\tilde{x}_{i}}-\frac{d x_{i}}{x_{i}}\right|_{g}=\mathscr{O}\left(x_{1}+x_{2}\right),
$$

and hence

$$
\left|\Phi^{*} \tilde{g}-g\right|_{g}=\mathscr{O}\left(x_{1}+x_{2}\right) .
$$

A weakly asymptotically product hyperbolic metric $g$ is sometimes also called weakly asymptotically Einstein, because of

Lemma 28. The metric (5.1) satisfies the estimate

$$
\left|\operatorname{Ric}^{g}+g\right|_{g}=\mathscr{O}\left(x_{1}+x_{2}\right) .
$$


The proof is deferred to the next subsection, where the formalism for the necessary calculations is developed.

Definition 27 fixes the behaviour of the metric $g$ in regular asymptotic directions (when both $x_{1}$ and $x_{2}$ tend to 0 ). As indicated earlier, we shall also define a narrower class of strongly asymptotically Einstein metrics, for which the Einstein tensor decays uniformly near the entire boundary of $M$, i.e. where $x_{1}$ or $x_{2}$ but not necessarily both tend to 0 . The goal in the next few subsections is to find the equations which the limiting values of the metric $g$ must satisfy at $x_{1}=0$ and at $x_{2}=0$, in order that $g$ lie in this smaller class.

Later in the paper, in $\$ 7.3$, we shall also define a class of 'near-product hyperbolic' metrics; these will be defined by slightly different conditions, but we show there that any strongly asymptotically Einstein metric is of near-product type.

This profusion of similar names is indicative of the fact that for metrics which are modelled by symmetric spaces of rank greater than one, it is by no means clear what the precise conditions are under which a metric should really be considered 'asymptotically symmetric'; each of the classes of metrics above has some claim to this moniker in the product hyperbolic setting.

\subsection{Asymptotic curvature calculations}

We now calculate the asymptotics of the Ricci curvature for a weakly asymptotically product hyperbolic metric (5.2) on $M_{1} \times M_{2}$. In the course of this the proof of Lemma 28 will emerge, as well as motivation for the extra conditions imposed on $g$ to warrant the name strongly asymptotically Einstein.

As before, assume that $M, X$ and the distributions $\mathscr{D}_{i} \subset \mathscr{F}_{i}$ are all of product type. The main calculations are local near the boundary faces; to be definite we work in the region where $x_{2} \rightarrow 0$, and write $g$ in the form

$$
g=g_{1}+\lambda_{2}\left(\frac{d x_{2}^{2}}{x_{2}^{2}}+\frac{\gamma_{2}}{4 x_{2}^{2}}+\frac{\eta_{2}^{2}}{4 x_{2}^{4}}\right),
$$

where the two terms are metrics along horizontal and vertical slices, $M_{1} \times\left\{p_{2}\right\}$ and $\left\{p_{1}\right\} \times M_{2}$, respectively. We assume that

- the $x_{2}$ dependence is only what is written explicitly; in other words, $g_{1}, \eta_{2}$ and $\gamma_{2}$ are defined and smooth on $M_{1} \times X_{2}$ and are independent of $x_{2}$;

- $\eta_{2}$ is the pullback of a contact form from $X_{2}$, hence is independent of $M_{1}$;

- $\gamma_{2}$ is a family of metrics on $\mathscr{D}_{2}$ compatible with $\eta_{2}$ (and hence gives a $G_{2}$-conformal structure on each slice $\left.\left\{p_{1}\right\} \times X_{2}\right)$.

The precise form of $g_{1}$ is not so important for the moment, but in order to maintain consistency with (5.2), we also impose that in analogous coordinates near the boundary of $M_{1}, g_{1} \sim \lambda_{1}\left(d x_{1}^{2} / x_{1}^{2}+\gamma_{1} / 4 x_{1}^{2}+\eta_{1}^{2} / 4 x_{1}^{4}\right)$ as $x_{1} \rightarrow 0$, and that $\gamma_{2}$ converges to a representative of the specified conformal class $\left[\gamma_{2}\right]$ as $x_{1} \rightarrow 0$. However, these last conditions do not enter into the immediate considerations. 
Well-known formulæ due to O'Neill (cf. Proposition 9.36 in [1]) express the Ricci curvature of a Riemannian submersion in terms of the Ricci curvatures of the base and fibres and two additional tensors: the second fundamental form $T$ of the fibres and another tensor which measures the deviation of the horizontal subspaces from being integrable. To adapt this to our setting, we regard $M$ as a fibration $M_{1} \times M_{2} \rightarrow M_{1}$. The two factors are orthogonal, and the horizontal subspaces are integrable (with leaves the $M_{1}$ slices, i.e. the submanifolds $M_{1} \times\left\{q_{2}\right\}$ ), but this is still not quite a Riemannian submersion because $g_{1}$ depends also on $M_{2}$. In the curvature computations below, however, it behaves asymptotically as $x_{2} \rightarrow 0$ like a Riemannian submersion: the negative powers of $x_{2}$ in all terms in $g_{2}$ add an extra $x_{2}$ factor to all derivatives in the $M_{2}$ directions.

We continue by defining the various quantities which appear in the O'Neill formulæ, and developing some of their properties. The first is the second fundamental form for $\gamma_{2}$. This is the section $T$ of $T^{*} M_{1} \otimes \operatorname{Sym}^{2}\left(\mathscr{D}_{2}^{*}\right)$ defined by

$$
\left\langle T\left(\xi_{2}, \zeta_{2}\right), \xi_{1}\right\rangle=-\frac{1}{2}\left(\mathscr{L}_{\xi_{1}} \gamma_{2}\right)\left(\xi_{2}, \zeta_{2}\right) \Leftrightarrow T=-\frac{1}{2} d^{M_{1}} \gamma_{2}
$$

(Here and later, a subscript 1 or 2 of a vector indicates the factor to which it is tangent.) For either of these expressions we regard $\gamma_{2}$ as a section of $\operatorname{Sym}^{2}\left(\mathscr{D}_{2}^{*}\right)$, which in turn is a trivial bundle over each $M_{1}$ slice. For each $\xi_{2} \in \mathscr{D}_{2}$ we also set

$$
T_{\xi_{2}} \in \operatorname{End}\left(\mathscr{D}_{2}, T M_{1}\right), \quad T_{\xi_{2}}^{*} \in \operatorname{End}\left(T M_{1}, \mathscr{D}_{2}\right)
$$

where the metrics $g_{1}$ and $\gamma_{2}$ are used to dualize.

There is still freedom in the choice of the representative $\gamma_{2} \in\left[\gamma_{2}\right]$, but we now fix the normalization that the volume form $d V^{\gamma_{2}}$ is constant in the $M_{1}$ directions. Consequently, the mean curvature vector vanishes:

$$
\operatorname{Tr}^{\gamma_{2}} T=0
$$

The trivial connection $d^{M_{1}}$ on $\mathscr{D}_{2}$ is not compatible with the metric, but to find one which is, it suffices to add the second map in (5.5); thus

$$
\left.\nabla=d^{M_{1}}+T \quad \text { (i.e. } \nabla_{\xi_{1}} \xi_{2}=d_{\xi_{1}}^{M_{1}} \xi_{2}+T_{\xi_{2}}^{*} \xi_{1}\right)
$$

defines a unitary connection on $\mathscr{D}_{2}$ over $M_{1}$. The divergence of $T$ with respect to this connection is the bilinear form on $\mathscr{D}_{2}$,

$$
\left(\delta^{M_{1}} T\right)\left(\xi_{2}, \zeta_{2}\right)=-\sum\left\langle\left(\nabla_{e_{\alpha}} T\right)\left(\xi_{2}, \zeta_{2}\right), e_{\alpha}\right\rangle,
$$

where $\left\{e_{\alpha}\right\}$ is an orthonormal frame for $T M_{1}$. The final ingredient we need is the bilinear form $Q$ on $T M_{1}$ defined by contracting the product of $T$ with itself in the $\operatorname{Sym}^{2}\left(\mathscr{D}_{2}^{*}\right)$ component with respect to $\gamma_{2}$,

$$
Q\left(\xi_{1}, \eta_{1}\right):=\left\langle T_{\xi_{1}}, T_{\eta_{1}}\right\rangle_{\gamma_{2}}
$$

Before proceeding, we derive the crucial first order properties of $T$. 
Lemma 29. Let $d_{\nabla}^{M_{1}}$ denote the exterior derivative on $M_{1}$ coupled to the connection $\nabla$ on $\mathscr{D}_{2}$. Then

$$
d_{\nabla}^{M_{1}} T=0 .
$$

Proof. By (5.6), $d_{\nabla}^{M_{1}} T=d^{M_{1}} T+T \wedge T$, where in the last term we regard the two factors as elements of $T^{*} M_{1} \otimes \operatorname{End}\left(\mathscr{D}_{2}\right)$ and $T^{*} M_{1} \otimes \operatorname{Sym}^{2}\left(\mathscr{D}_{2}\right)$, respectively. The second expression for $T$ in (5.4) gives $d^{M_{1}} T=0$, hence it suffices to prove

$$
T \wedge T=0 \text {. }
$$

This identity in turn is a direct consequence of the symmetry of the action

$$
(u \cdot q)(x, y)=q(u x, y)+q(x, u y)=\gamma_{2}((u v+v u) x, y)
$$

of a symmetric endomorphism $u$ on a quadratic form $q(x, y)=\gamma_{2}(v x, y)$ in the pair $(u, v)$.

Lemma 30. On each slice $M_{1} \times\left\{z_{2}\right\}$ there is a Bianchi identity of the form

$$
\left(\delta^{M_{1}} Q+\frac{1}{2} d \operatorname{Tr} Q\right)_{\xi}=\left\langle\delta^{M_{1}} T, T^{*} \xi\right\rangle, \quad \xi \in T M_{1} .
$$

Proof. Choose an orthonormal frame $\left\{e_{\alpha}\right\}$ for $T M_{1}$ and extend $\xi$ to a vector field on $M_{1}$ which is parallel with respect to $\nabla$ at some point $z_{1}$. Then, calculating at $z_{1}$,

$$
\begin{aligned}
\left(\delta^{M_{1}} Q\right)(\xi) & =-\sum_{\alpha} \nabla_{e_{\alpha}} Q\left(e_{\alpha}, \xi\right)=-\sum_{\alpha}\left\langle\left(\nabla_{e_{\alpha}} T^{*}\right) e_{\alpha}, T^{*} \xi\right\rangle+\left\langle T^{*} e_{\alpha},\left(\nabla_{e_{\alpha}} T^{*}\right) \xi\right\rangle \\
& =\left\langle\delta^{M_{1}} T, T^{*} \xi\right\rangle-\sum_{\alpha}\left\langle T^{*} e_{\alpha},\left(\nabla_{\xi} T^{*}\right)\left(e_{\alpha}\right)\right\rangle=\left\langle\delta^{M_{1}} T, T^{*} \xi\right\rangle-\frac{1}{2} d \operatorname{Tr} Q(\xi) .
\end{aligned}
$$

The second equality uses that $d_{\nabla}^{M_{1}} T=0$.

There is also a second fundamental form $\mathbb{I}_{i}$ and corresponding mean curvature vector $N_{i}=\operatorname{Tr}^{g_{i}} \mathbb{I}_{i}$ for each $M_{i}$ slice, $i=1,2$. Note that $N_{2}$ is different from $\operatorname{Tr}^{\gamma_{2}} T$ (which we are assuming is equal to 0 ), since in the latter one only takes the trace in the $\mathscr{D}_{2}$ directions.

We can now state an exact formula for the Ricci curvature.

Lemma 31. Let $\left(M=M_{1} \times M_{2}, g=g_{1}+g_{2}\right)$ be a metric on $M$ keeping the factors $M_{1}$ and $M_{2}$ orthogonal. Let $\mathbb{I}_{i}$ be the second fundamental form of $M_{i}$ and $N_{i}$ the mean curvature vectors. Then the Ricci tensor of $g$ is given by

$$
\begin{aligned}
\operatorname{Ric}^{g}\left(\xi_{1}, \zeta_{1}\right)= & \operatorname{Ric}^{g_{1}}\left(\xi_{1}, \zeta_{1}\right)-\left(\delta^{M_{2}} \mathbb{I}_{1}\right)\left(\xi_{1}, \zeta_{1}\right)-\left\langle\mathbb{I}_{1}\left(\xi_{1}, \zeta_{1}\right), N_{1}\right\rangle \\
& +\left(\delta^{M_{1}}\right)^{*} N_{2}\left(\xi_{1}, \zeta_{1}\right)-\left\langle\mathbb{I}_{2}^{*} \xi_{1}, \mathbb{I}_{2}^{*} \zeta_{1}\right\rangle,
\end{aligned}
$$

with an analogous expression for the restriction of $\mathrm{Ric}^{g}$ to $T M_{2}$, where $\delta^{*}$ is the symmetrization of the covariant derivative; and

$$
\begin{aligned}
\operatorname{Ric}^{g}\left(\xi_{1}, \xi_{2}\right)= & \left\langle\delta^{M_{1}} \mathbb{I}_{1}\left(\xi_{1}\right), \xi_{2}\right\rangle+\left\langle\nabla_{\xi_{1}} N_{1}, \xi_{2}\right\rangle \\
& +\left\langle\delta^{M_{2}} \mathbb{I}_{2}\left(\xi_{2}\right), \xi_{1}\right\rangle+\left\langle\nabla_{\xi_{2}} N_{2}, \xi_{1}\right\rangle,
\end{aligned}
$$

where $\delta^{M_{i}} \mathbb{I}_{i}$ is the divergence of $\mathbb{I}_{i}$ regarded as a symmetric 2-tensor along $M_{i}$.

The derivations of these two formulæ are left to the reader. 
As a first application, we have

Proof of Lemma 28. We will be applying (5.8) and (5.9) with $M_{i}=(0,1)_{x_{i}} \times X_{i}$. The second fundamental form of the slices $M_{1} \times\left\{p_{2}\right\}$ is

$$
\left\langle\mathbb{I}_{1}, \xi_{2}\right\rangle=-\frac{1}{2} \mathscr{L}_{\xi_{2}} g_{1}=-\frac{\lambda_{1}}{2}\left(\frac{1}{4 x_{1}^{2}} \mathscr{L}_{\xi_{2}} \gamma_{1}+\frac{1}{4 x_{1}^{4}} \mathscr{L}_{\xi_{2}} \eta_{1}^{2}\right) .
$$

If $\xi_{2}$ is a unit vector in $T X_{2}$, then

$$
\frac{1}{4 x_{1}^{2}} \mathscr{L}_{\xi_{2}} \gamma_{1}+\frac{1}{4 x_{1}^{4}} \mathscr{L}_{\xi_{2}} \eta_{1}^{2}=\mathscr{O}\left(x_{2}\right),
$$

since $\mathscr{D}_{1}$ does not depend on $M_{2}$. Hence on each slice $M_{1} \times\left\{x_{2}\right\}, \mathbb{I}_{1}=\mathscr{O}\left(x_{2}\right)$, and the same is true for all its derivatives. On these same slices one also has

$$
\operatorname{Ric}^{g_{1}}=-\lambda_{1} g_{1}+\mathscr{O}\left(x_{1}\right) .
$$

Analogously, on the slices $\left\{p_{1}\right\} \times M_{2}$, we have

$$
\mathbb{I}_{2}=\mathscr{O}\left(x_{1}\right) \text { and } \quad \operatorname{Ric}^{g_{2}}=-\lambda_{2} g_{2}+\mathscr{O}\left(x_{2}\right) .
$$

Inserting these in (5.8) and (5.9) gives $\operatorname{Ric}^{g}=-g+\mathscr{O}\left(x_{1}+x_{2}\right)$, as desired.

The main result of this subsection is

Lemma 32. Let $g$ be defined by (5.3), and suppose that

$$
\left\{\begin{array}{l}
\delta^{M_{1}} T=0 \\
\operatorname{Ric}^{g_{1}}\left(\xi_{1}, \zeta_{1}\right)+\lambda_{1}\left\langle\xi_{1}, \zeta_{1}\right\rangle_{g_{1}}=\left\langle T^{*} \xi_{1}, T^{*} \zeta_{1}\right\rangle_{\gamma_{2}},
\end{array}\right.
$$

for all vectors $\xi_{1}, \zeta_{1} \in T M_{1}$. Then $\mathrm{Ric}^{g}=-g+\mathscr{O}\left(x_{2}\right)$.

The point in this lemma is that the error term $\mathscr{O}\left(x_{2}\right)$ does not depend on $x_{1}$, so the metric $g$ is asymptotically Einstein not only in regular directions, but also when one goes to the face $x_{2}=0$. Doing the same along the other face will lead to the notion of 'strongly asymptotically Einstein'.

Remark 33. Note that the right hand side of the Bianchi identity in Lemma 30 vanishes when the first equation in (5.10) is satisfied.

Proof. We apply (5.8) and (5.9) as follows. First, just as before, $\mathbb{I}_{1}=\mathscr{O}\left(x_{2}\right)$, and hence $N_{1}=\mathscr{O}\left(x_{2}\right)$ too. On the other hand, the normalization on the volume form implies that $N_{2}=0$, thus

$$
\begin{aligned}
& \operatorname{Ric}^{g}\left(\xi_{1}, \zeta_{1}\right)=\operatorname{Ric}^{g_{1}}\left(\xi_{1}, \zeta_{1}\right)-\left\langle\mathbb{I}_{2}^{*} \xi_{1}, \mathbb{I}_{2}^{*} \zeta_{1}\right\rangle+\mathscr{O}\left(x_{2}\right), \\
& \operatorname{Ric}^{g}\left(\xi_{1}, \xi_{2}\right)=\mathscr{O}\left(x_{2}\right), \\
& \operatorname{Ric}^{g}\left(\xi_{2}, \zeta_{2}\right)=\operatorname{Ric}^{g_{2}}\left(\xi_{2}, \zeta_{2}\right)-\left(\delta^{M_{1}} \mathbb{I}_{2}\right)\left(\xi_{2}, \zeta_{2}\right)+\mathscr{O}\left(x_{2}\right) .
\end{aligned}
$$


From the formula (5.3) and the fact that $\eta_{2}$ is constant along $M_{1}$ slices, we get

$$
\mathbb{I}_{2}=T / x_{2}^{2},
$$

and since the connection (5.6) on $\mathscr{D}_{2}$ along $M_{1}$ is exactly the one induced by the LeviCivita connection of $g$, the result follows.

To conclude the section, observe that the formulæ in the lemma correspond exactly to the standard formulæ obtained for a Riemannian submersion with integrable horizontal distribution [1, Proposition 9.36], as expected from our claim that the asymptotic behaviour when $x_{2} \rightarrow 0$ is that of a Riemannian submersion.

\section{Extending the approximate solution to the codimension one boundary faces}

Let $\left(M=M_{1} \times M_{2}, g=g_{1}^{0}+g_{2}^{0}\right)$ be a product of A $\mathbb{K} H$ Einstein metrics, with conformal infinity $\mathfrak{c}^{0}=\left(\mathscr{D}_{1}^{0}, \mathscr{D}_{2}^{0},\left[\gamma_{1}^{0}\right],\left[\gamma_{2}^{0}\right]\right)$ on $X=X_{1} \times X_{2}$. As in the last section, we consider deformations $\mathfrak{c}$ of $\mathfrak{c}^{0}$ (assumed to be globally integrable in case either $X_{1}$ or $X_{2}$ is not simply connected). According to Lemma 25 , the pair of distributions $\mathscr{D}_{1}, \mathscr{D}_{2}$ remains of product type on $X$, and we then extend these by pullback to a pair of transverse distributions of product type on all of $M$. Choose metrics $\gamma_{i}$ representing each of the conformal classes $\left[\gamma_{i}\right]$. In the real or complex case, these may depend on both factors of $X$, but we maintain the normalization so that, still just over $X, d V^{\gamma_{1}}$ is independent of $X_{2}$, and similarly $d V^{\gamma_{2}}$ is independent of $X_{1}$. Based on the calculations of $\S 5.3$, we now address the problem of how to extend $\left(\gamma_{1}, \gamma_{2}\right)$ over the faces $M_{1} \times X_{2}$ and $X_{1} \times M_{2}$ to obtain a metric which is strongly asymptotically Einstein.

\subsection{Extension along boundary faces}

We focus on the extension of $\gamma_{1}$ over $M_{1} \times X_{2}$, since the other case is treated exactly the same.

Let us restate the problem more carefully. On the face $M_{1} \times X_{2}$, we seek metrics $g_{1}$ on $T M_{1}$ and $\tilde{\gamma}_{2}$ on $\mathscr{D}_{2}$ which solve the system (5.10). The solutions are constrained by the requirements that $d V \tilde{\gamma}_{2}$ is independent of $M_{1}$, and that $\left(g_{1}, \tilde{\gamma}_{2}\right)$ is asymptotic at $X=$ $\partial M_{1} \times X_{2}$ to the given $\left(G_{1} \times G_{2}\right)$-conformal structure in the sense that

$$
g_{1} \sim \lambda_{1}\left(\frac{d x_{1}^{2}}{x_{1}^{2}}+\frac{\gamma_{1}}{4 x_{1}^{2}}+\frac{\eta_{1}^{2}}{4 x_{1}^{4}}\right), \quad \tilde{\gamma}_{2} \sim \gamma_{2},
$$

as $x_{1} \rightarrow 0$ (with an error term $\mathscr{O}\left(x_{1}^{v}\right)$ for some $v>0$ ).

Lemma 34. Suppose that $\mathbb{K}_{2}=\mathbb{H}$ or $\mathbb{O}$. Then (5.10) reduces to a single uncoupled equation on $M_{1}$ which is simply the usual Einstein equation. 
Proof. Under this hypothesis, $\gamma_{2}$ is independent of the $X_{1}$ factor. Hence $T \equiv 0$ and the first equation in (5.10) is satisfied. The second equation reduces to the uncoupled Einstein equation on $M_{1}$. By Proposition 22 we can extend the conformal class [ $\left.\gamma_{1}\right]$ on $X_{1}$ to an $\mathrm{A} \mathbb{K}_{1} \mathrm{H}$ Einstein metric $g_{1}$ on $M_{1}$; note that this is actually done parametrically, depending on $q_{2} \in X_{2}$.

When $M_{2}$ is real or complex, (5.10) cannot be reduced in this way, but fortunately, solutions can still be obtained near to the standard one by perturbation methods.

We can now state and prove the main result of this section.

Theorem 35. Suppose $M=M_{1} \times M_{2}$ is a product of $A \mathbb{K} H$ Einstein spaces such that the $L^{2}$ nullspace for the linearized gauged Einstein operator on $M_{1}$ vanishes. Then, for any small globally integrable perturbation of the product $\left(G_{1} \times G_{2}\right)$-conformal structure on $X$, the system (5.10) has a global solution $\left(g_{1}, \tilde{\gamma}_{2}\right)$ on the face $M_{1} \times X_{2}$ with the prescribed asymptotic behaviour at $X$, more precisely on each slice $M_{1} \times\left\{q_{2}\right\}$,

$$
g_{1}-\lambda_{1}\left(\frac{d x_{1}^{2}}{x_{1}^{2}}+\frac{\gamma_{1}}{4 x_{1}^{2}}+\frac{\eta_{1}^{2}}{4 x_{1}^{4}}\right) \in x_{1}^{v} \Lambda^{2, \alpha}, \quad \tilde{\gamma}_{2}-\gamma_{2} \in x_{1}^{v} \Lambda^{2, \alpha},
$$

with smooth dependence with respect to $q_{2}$.

Proof. This proof is similar to that for Proposition 22. Consider the slice $M_{1} \times\left\{q_{2}\right\}$, and begin with the conformal structures $\gamma_{1}, \gamma_{2}$ on $X_{1} \times X_{2}$, with $d V^{\gamma_{2}}$ independent of $X_{1}$. Fix a smooth extension map assigning to $\gamma_{1}$ a metric $g_{1}$ on $M_{1}$ with

$$
g_{1}=\lambda_{1}\left(\frac{d x_{1}^{2}}{x_{1}^{2}}+\frac{\gamma_{1}}{4 x_{1}^{2}}+\frac{\eta_{1}^{2}}{4 x_{1}^{4}}\right)+k, \quad k \in x_{1}^{\nu} \Lambda^{2, \alpha},
$$

as $x_{1} \rightarrow 0$. Here $\Lambda^{2, \alpha}$ is the geometric Hölder space on $M_{1} \times\left\{q_{2}\right\}$. The weight $v$ is positive; we can fix $v=1$, but any smaller value is possible. Recall that we already have

$$
\operatorname{Ric}^{g_{1}}+g_{1}=\mathscr{O}\left(x_{1}\right) .
$$

As before, extend $\gamma_{2}$ by pullback on $M_{1}$, so that the corresponding second fundamental form $T=-\frac{1}{2} d^{M_{1}} \tilde{\gamma}_{2}$ satisfies also

$$
T=\mathscr{O}\left(x_{1}\right)
$$

When $\mathbb{K}=\mathbb{R}$, we consider perturbations $\phi$ of $\gamma_{2}$ which fix $d V^{\gamma_{2}}$, so the tangent space consists of trace-free symmetric 2-tensors, i.e. sections of

$$
\mathscr{S}=\operatorname{Sym}_{0}^{2}\left(T X_{2}\right) \text {. }
$$

When $\mathbb{K}=\mathbb{C}, \tilde{\gamma}_{2}(\cdot, \cdot)=d \eta_{2}(\cdot, J \cdot)$, where $J$ is an almost complex structure; the perturbation $\phi$ must also remain compatible with $d \eta_{2}$ on $\mathscr{D}_{2}$, or equivalently is a deformation of $J$, so the tangent space consists of trace-free $J$-skew-Hermitian symmetric 2-tensors, i.e. sections of

$$
\mathscr{S}=\operatorname{Sym}_{0}^{-}\left(\mathscr{D}_{2}\right)
$$


Consider elements

$$
h \in \Lambda_{v}^{2, \alpha}\left(M_{1} \times\left\{q_{2}\right\}, \operatorname{Sym}^{2}\left(T M_{1}\right)\right), \quad \phi \in \Lambda_{v}^{2, \alpha}\left(M_{1} \times\left\{q_{2}\right\}, \mathscr{S}\right),
$$

and assume that both $h$ and $\phi$ have sufficiently small norm. Suppose that $\left(g_{1}+h, \tilde{\gamma}_{2}+\phi\right)$ is a solution of the system (5.10) along $M_{1}$.

Denote by $T^{\phi}$ and $Q^{\phi}$ the second fundamental form and corresponding quadratic form defined by $\gamma_{2}+\phi$. To break the diffeomorphism invariance of the equation, we add the Bianchi gauge condition

$$
B^{g_{1}}(h)=\left(\delta^{g_{1}}+\frac{1}{2} d \operatorname{Tr}^{g_{1}}\right) h=0 .
$$

Thus we consider the system

$$
\Phi^{g_{1}, \tilde{\gamma}_{2}}(h, \phi):=\left(\operatorname{Ric}^{g_{1}+h}-\lambda\left(g_{1}+h\right)-Q^{\phi}+\left(\delta^{g_{1}+h}\right)^{*} B^{g_{1}}(h), \delta^{g_{1}+h, \phi} T^{\phi}\right)=0 .
$$

By Lemma 30, any solution of this equation must also satisfy

$$
B^{g_{1}+h}\left(\delta^{g_{1}+h}\right)^{*} B^{g_{1}}(h)=0 .
$$

By the same argument as in the uncoupled case (cf. Proposition 19), we conclude that $B^{g_{1}} h=0$. Hence a solution of $\Phi^{g_{1}, \tilde{\gamma}_{2}}(h, \phi)=0$ is also a solution of the original system (5.10).

As in the proof of Proposition 22, it suffices to check that

$$
\Phi^{g_{1}, \tilde{\gamma}_{2}}: \Lambda_{v}^{2, \alpha}\left(M_{1} \times\left\{q_{2}\right\}, \operatorname{Sym}^{2}\left(T M_{1}\right) \oplus \mathscr{S}\right) \rightarrow \Lambda_{v}^{0, \alpha}\left(M_{1} \times\left\{q_{2}\right\}, \operatorname{Sym}^{2}\left(T M_{1}\right) \oplus \mathscr{S}\right)
$$

is a $\mathscr{C}^{1}$ mapping of Banach spaces, for $(h, \phi)$ of sufficiently small norm, and furthermore, that its linearization

$$
\begin{aligned}
\left.D \Phi^{g_{1}, \tilde{\gamma}_{2}}\right|_{(0,0)}: \Lambda_{v}^{2, \alpha}\left(M_{1} \times\left\{q_{2}\right\}, \operatorname{Sym}^{2}\left(T M_{1}\right) \oplus \mathscr{S}\right) & \\
& \rightarrow \Lambda_{v}^{0, \alpha}\left(M_{1} \times\left\{q_{2}\right\}, \operatorname{Sym}^{2}\left(T M_{1}\right) \oplus \mathscr{S}\right)
\end{aligned}
$$

is an isomorphism at the product metric. Since the linearization of the Bianchi-gauged Einstein equation is $\nabla^{*} \nabla-2 \stackrel{\circ}{R}$, and $T=0$ at the product metric, this linearization decouples as

$$
\left.D \Phi\right|_{(0,0)}(\dot{h}, \dot{\phi})=\left(\left(\nabla^{*} \nabla-2 \stackrel{R}{R} \dot{h}, \nabla^{*} \nabla \dot{\phi}\right)\right.
$$

for the metric $g_{1}^{0}$. By the hypothesis on the vanishing of the $L^{2}$ nullspace for $\left(M_{1}, g_{1}\right)$, the first component is an isomorphism. The second component $\nabla^{*} \nabla$ is the rough Laplacian, and this is an isomorphism for weights $v \in(0, n+d-1)$.

The last statement comes from the smooth dependence of the solution constructed by the inverse function theorem with respect to the parameter $q_{2}$. 
Corollary 36. With the same hypotheses as in Theorem 35 , let $\left(g_{1}, \tilde{\gamma}_{2}\right)$ be the solution of the system (5.10) on the face $M_{1} \times X_{2}$. Then the metric

$$
h=g_{1}+\lambda_{2}\left(\frac{d x_{2}^{2}}{x_{2}^{2}}+\frac{\tilde{\gamma}_{2}}{4 x_{2}^{2}}+\frac{\eta_{2}^{2}}{4 x_{2}^{4}}\right),
$$

defined in some neighbourhood of the face $M_{1} \times X_{2}$ where $x_{2} \ll 1$ on $M_{1} \times M_{2}$, satisfies:

1. $\operatorname{Ric}^{h}+h=O\left(x_{2}\right)$ uniformly on the closure of this face, and more precisely $\operatorname{Ric}^{h}+h$ $\in x_{2} \Lambda^{\alpha}$

2. when $x_{1} \rightarrow 0$, then $h-h_{0} \in x_{1} \Lambda^{2, \alpha}$, where $h_{0}$ is the model metric (5.1) given by the formula

$$
h_{0}=\lambda_{1}\left(\frac{d x_{1}^{2}}{x_{1}^{2}}+\frac{\gamma_{1}}{4 x_{1}^{2}}+\frac{\eta_{1}^{2}}{4 x_{1}^{4}}\right)+\lambda_{2}\left(\frac{d x_{2}^{2}}{x_{2}^{2}}+\frac{\gamma_{2}}{4 x_{2}^{2}}+\frac{\eta_{2}^{2}}{4 x_{2}^{4}}\right) .
$$

Proof. This is a direct consequence of the formulæ (5.8) and (5.9) for Ric ${ }^{g}$, since the solution $\left(g_{1}, \gamma_{2}\right)$ depends smoothly on the parameter $q_{2} \in X_{2}$.

We conclude this section with a comment about regularity. Exactly as in Remark 23 at the very end of $\S 4$, the solutions obtained in Theorem 35 are polyhomogeneous at the boundaries of the codimension one faces provided the $\left(G_{1} \times G_{2}\right)$-conformal infinity data on $X$ is smooth. The proof is identical to the one for the uncoupled $\mathrm{A} \mathbb{K} \mathrm{H}$ Einstein equations.

\subsection{Strongly asymptotically Einstein metrics}

We are now ready to define, given any small deformation $\mathfrak{c}$ of the given $\left(G_{1} \times G_{2}\right)$ conformal structure $\mathrm{c}^{0}$ on $X$, a global, approximately Einstein metric $g$ on $M$. By Theorem 35 and Corollary 36, we extend the data $\left(\gamma_{1}, \gamma_{2}\right)$ on the two faces $F_{1}=M_{1} \times X_{2}$ and $F_{2}=X_{1} \times M_{2}$ to get pairs $\left(g_{1}, \tilde{\gamma}_{2}\right)$ and $\left(\tilde{\gamma}_{1}, g_{2}\right)$ solving the system (5.10) on each face. Therefore, the two metrics on $M$,

$$
h_{1}=g_{1}+\lambda_{2}\left(\frac{d x_{2}^{2}}{x_{2}^{2}}+\frac{\tilde{\gamma}_{2}}{4 x_{2}^{2}}+\frac{\eta_{2}^{2}}{4 x_{2}^{4}}\right), \quad h_{2}=\lambda_{1}\left(\frac{d x_{1}^{2}}{x_{1}^{2}}+\frac{\tilde{\gamma}_{1}}{4 x_{1}^{2}}+\frac{\eta_{1}^{2}}{4 x_{1}^{4}}\right)+g_{2}
$$

defined in the neighbourhoods $x_{2} \ll 1$ and $x_{1} \ll 1$ of $F_{1}$ and $F_{2}$, respectively, satisfy

$$
h_{1}-h_{0} \in x_{1} \Lambda^{2, \alpha}, \quad h_{2}-h_{0} \in x_{2} \Lambda^{2, \alpha},
$$

where

$$
h_{0}=\lambda_{1}\left(\frac{d x_{1}^{2}}{x_{1}^{2}}+\frac{\gamma_{1}}{4 x_{1}^{2}}+\frac{\eta_{1}^{2}}{4 x_{1}^{4}}\right)+\lambda_{2}\left(\frac{d x_{2}^{2}}{x_{2}^{2}}+\frac{\gamma_{2}}{4 x_{2}^{2}}+\frac{\eta_{2}^{2}}{4 x_{2}^{4}}\right)
$$

is the initial model metric (5.1). It remains to glue $h_{1}$ and $h_{2}$ in the region $\left\{x_{1} \ll 1, x_{2} \ll 1\right\}$ where they both exist. Choose some cutoff function $\chi$ such that $\chi(x)=1$ for $x<1 / 2$ and $\chi(x)=0$ for $x>2$, and consider the metric

$$
h=\chi\left(\frac{x_{2}}{x_{1}}\right) h_{1}+\left(1-\chi\left(\frac{x_{2}}{x_{1}}\right)\right) h_{2},
$$


now defined in a neighbourhood of the whole boundary $F_{1} \cup F_{2}$. In the region $1 / 2<$ $x_{2} / x_{1}<2$, all the derivatives of $\chi\left(x_{2} / x_{1}\right)$ remain bounded for the metric $d x_{1}^{2} / x_{1}^{2}+$ $d x_{2}^{2} / x_{2}^{2}$, hence in that region $h-h_{0} \in x_{1} \Lambda^{2, \alpha}$ (or equivalently $x_{2} \Lambda^{2, \alpha}$ ), and therefore $\mathrm{Ric}^{h}+h=\mathscr{O}\left(x_{1}\right)$, or more precisely $\mathrm{Ric}^{h}+h \in x_{1} \Lambda^{2, \alpha}$. Globally, in a neighbourhood of $F_{1} \cup F_{2}$,

$$
\operatorname{Ric}^{h}+h \in x_{1}^{v} x_{2}^{v} \Lambda^{\alpha}, \quad v=1 / 2 .
$$

We now generalize this model. Fix $0<v \leq 1 / 2$.

Definition 37. A metric $g$ on $M$ is strongly asymptotically Einstein if it differs from the metric $h$ defined above by a term in $x_{1}^{\nu} x_{2}^{\nu} \Lambda^{2, \alpha}$.

In particular, the Ricci curvature of any such metric satisfies (6.1). Note too that any other reasonably method of patching $h_{1}$ and $h_{2}$ together near the corner yields a metric $h^{\prime}$ which is strongly asymptotically Einstein in this same sense.

\section{Generalized Laplacians on near-product hyperbolic spaces}

We now discuss the construction of a parametrix for $L^{g}$ when $g$ is a strongly asymptotically Einstein perturbation of a product hyperbolic metric. Our goal is to show that $L^{g}$ is invertible between two weighted Hölder spaces. We do this in the following steps. First, we analyze the Schwartz kernel of the inverse of $L^{g}$ when $(M, g)$ is exactly product hyperbolic using a contour integral representation; we go on to obtain conormal bounds for this Schwartz kernel on the 'product hyperbolic double space' $M_{\mathrm{ph}}^{2}$. This serves as an ansatz for the parametrix of $L^{g}$ when the metric $g$ is weakly asymptotically product hyperbolic. We introduce a stronger condition on $g$ of being near-product hyperbolic, and show that under this hypothesis we can construct a parametrix with Schwartz kernel conormal on $M_{\mathrm{ph}}^{2}$ which is an inverse of $L^{g}$ up to a compact error term. The final step is to show that each of these operators is bounded between weighted Hölder spaces, which implies that $L^{g}$ is Fredholm on these spaces. The fact that when $g$ is a product we have an exact inverse for $L^{g}$ on $L^{2}$ which is bounded between these Hölder spaces shows that $L^{g}$ is then invertible between these spaces. The parametrix construction varies continuously with $g$, so we conclude that $L^{g}$ remains invertible when $g$ is near-product hyperbolic and sufficiently close to a product metric.

\subsection{The inverse of $L$ when $g$ is a product}

We begin with an examination of the structure of the inverse of $L^{g}$ on $L^{2}(M)$ when $(M, g)$ is a product of $\mathrm{A} \mathbb{K H}$ spaces. This is mostly a review of the analysis in [21]. We first present a contour integral representation for the $L^{2}$ inverse $G$ of $L^{g}$ involving the resolvent families of the operators $L^{g_{j}}$ on each factor; from this we deduce estimates for the pointwise off-diagonal behaviour of the Schwartz kernel of $G$ using the analogous estimates for the Schwartz kernels of the two constituent resolvents. We do not make an effort to obtain the most precise pointwise estimates on $G$ here, but see [21] and [14] for more on this. 


\section{A representation formula for the Green function}

Let $(M, g)$ be a product of two $\mathrm{A} \mathbb{K} H$ Einstein spaces, with linearized gauged Einstein operator $L^{g}=\nabla^{*} \nabla-2 \stackrel{\circ}{R}$, acting on sections of

$$
\operatorname{Sym}^{2}\left(T^{*} M\right)=\operatorname{Sym}^{2}\left(T^{*} M_{1}\right) \oplus\left(T^{*} M_{1} \otimes T^{*} M_{2}\right) \oplus \operatorname{Sym}^{2}\left(T^{*} M_{2}\right) .
$$

The operator $L^{g}$ preserves the three summands and acts by

$$
L^{g_{1}}+\left(\nabla^{M_{2}}\right)^{*} \nabla^{M_{2}}, \quad\left(\nabla^{M_{1}}\right)^{*} \nabla^{M_{1}}+\left(\nabla^{M_{2}}\right)^{*} \nabla^{M_{2}}, \quad\left(\nabla^{M_{1}}\right)^{*} \nabla^{M_{1}}+L^{g_{2}},
$$

respectively. In each of these three cases, it has the form

$$
L^{g}=L_{1} \otimes I_{B_{2}}+I_{B_{1}} \otimes L_{2}
$$

where $L_{i}$ acts on a Banach space $B_{i}$ of sections of a bundle $E_{i}$ on $M_{i}$, and $L^{g}$ acts on the completed tensor product $B_{1} \widehat{\otimes} B_{2}$ of sections of $E=E_{1} \otimes E_{2}$ on $M$.

For the moment, let $B_{i}=L^{2}\left(M_{i}, E_{i} ; d V_{g_{i}}\right)$. Denote by $R_{i}(\mu)$ the resolvent family $\left(L_{i}-\mu\right)^{-1}$. This is a holomorphic family of bounded operators on $B_{i}$ for $\mu$ in the resolvent set $\mathbb{C} \backslash \operatorname{spec}\left(L_{i}\right)$; according to Theorem 15 ,

$$
\operatorname{spec}\left(L_{i}\right)=\left\{\lambda_{i j}\right\}_{j=1}^{N_{i}} \cup\left[\alpha_{i}, \infty\right)
$$

for some $\alpha_{i}>0$, with $\lambda_{i j} \in \mathbb{R}, \lambda_{i j}<\alpha_{i}$. Nondegeneracy of $\left(M_{i}, g_{i}\right)$ for the Einstein problem is the assumption that $0 \notin\left\{\lambda_{i j}\right\}$.

The resolvent family of the product operator, $R(\lambda)=(L-\lambda)^{-1}$, can be expressed as a sort of convolution of the resolvents on the two factors. More precisely, it is proved in [21] that

$$
R(\lambda)=-\frac{1}{2 \pi i} \int_{\Gamma_{\lambda}} R_{1}(\mu) R_{2}(\lambda-\mu) d \mu,
$$

where $\Gamma_{\lambda}$ is a contour lying in the common region of holomorphy of the two factors in the integrand with ends converging linearly to $\pm i \infty$ and such that the spectrum of $L_{1}$ lies entirely on one side and the spectrum of $L_{2}$ lies entirely on the other side. For us it suffices to take $\lambda=0$. In the simplest situation, neither $L_{1}$ nor $L_{2}$ has any negative eigenvalues, and in this case we take $\Gamma_{0}=i \mathbb{R}$. The general case, where one or the other does have such eigenvalues, requires a slight modification to this formula.

Setting $R(0)=L^{-1}$, and $G$ its Schwartz kernel, if $f \in \mathscr{C}_{0}^{\infty}(M)$, then $u=G f$ is the unique $L^{2}$ solution to the equation $L u=f$.

\section{Estimates on $G$}

Fix local coordinates $z_{j}=\left(x_{j}, y_{j}\right)$ near a boundary point of $M_{j}$, and denote by $K_{j}\left(z_{j}, z_{j}^{\prime}, \mu\right)$ the Schwartz kernel of $R_{j}(\mu)$. Also, replace $\mu$ by $i \mu$. Then (assuming neither $L_{j}$ has negative eigenvalues), the Schwartz kernel of $G$ equals

$$
G\left(z_{1}, z_{2}, z_{1}^{\prime}, z_{2}^{\prime}\right)=\frac{1}{2 \pi} \int_{-\infty}^{\infty} K_{1}\left(z_{1}, z_{1}^{\prime}, i \mu\right) K_{2}\left(z_{2}, z_{2}^{\prime},-i \mu\right) d \mu .
$$


We now obtain pointwise estimates for $G$ using the bounds on $K_{j}\left(z_{j}, z_{j}^{\prime}, i \mu\right)$ in Proposition 17. Our goal is to prove that $G$ lifts to a conormal distribution on a certain blowup of $M^{2}$ which is conormal at all boundaries, polyhomogeneous along the lifted diagonal, and has a leading polyhomogeneous term at the front faces with conormal remainder.

First observe that the integral (7.2) converges in the Banach space of bounded operators on $L^{2}\left(M ; d V_{g}\right)$. This follows from the elementary estimate

$$
\left\|R_{j}(i \mu)\right\|_{\mathscr{B}\left(L^{2}\left(M_{j}\right)\right)} \leq 1 /|\mu|
$$

which is a direct consequence of the spectral theorem for the selfadjoint operator $L_{j}$. This already uniquely specifies $G$ as an element of $\mathscr{D}^{\prime}\left(M^{2}\right)$.

Next, introduce the product hyperbolic double space

$$
M_{\mathrm{ph}}^{2}=\left(M_{1}\right)_{\mathbb{K}_{1} \Theta}^{2} \times\left(M_{2}\right)_{\mathbb{K}_{2} \Theta}^{2} ;
$$

this manifold with corners is simply the product of the $\mathbb{K}_{j} \Theta$ double spaces of the two factors. It has six codimension one boundary faces: the two front faces, which are the boundary hypersurfaces intersecting the lifted diagonal $\operatorname{diag}_{\mathrm{ph}}$ :

$$
\mathrm{ff}_{1}=\operatorname{ff}\left(\left(M_{1}\right)_{\mathbb{K}_{1} \Theta}^{2}\right) \times\left(M_{2}\right)_{\mathbb{K}_{2} \Theta}^{2} \quad \text { and } \quad \mathrm{ff}_{2}=\left(M_{1}\right)_{\mathbb{K}_{1} \Theta}^{2} \times \operatorname{ff}\left(\left(M_{2}\right)_{\mathbb{K}_{2} \Theta}^{2}\right),
$$

and the four side faces, which are the products of the side faces of one factor with the interior of the other factor. We denote the side faces by $B_{10, j}$ and $B_{01, j}, j=1,2$, and for uniformity also write $\mathrm{ff}_{j}=B_{11, j}$; the $j$ signifies that the face in question comes from a boundary face in the $M_{j}$ factor. Defining functions for any one of these faces will be written $\rho_{p q, j}, j=1,2$ and $p q=10,01,11$.

Continuing on with this notation, an index set $\sigma$ on $M_{\mathrm{ph}}^{2}$ is a tuple of real numbers $\left(\sigma_{p q, j}\right)$, where $\sigma_{p q, j}$ indicates a rate of conormal decay at the boundary face $B_{p q, j}$.

We shall prove that $G$ is conormal at all boundary faces of $M_{\mathrm{ph}}^{2}$ and along the lifted diagonal. The conormal estimates here are not quite sharp; there is a subtle cancellation in (7.2), explored more carefully in [21], which leads to vanishing rates which are slightly better (by a logarithmic factor), but this is not needed here.

Proposition 38. The lift of the Schwartz kernel $G$ to the space $M_{\mathrm{ph}}^{2}$ is an element of $\mathscr{A}_{\mathrm{ff}}^{\sigma}\left(M_{\mathrm{ph}}^{2}, \operatorname{diag}_{\mathrm{ph}}\right)$, where $\sigma=\left(\sigma_{p q, j}\right)$ is the index set with $\sigma_{10, j}=\sigma_{01, j}=n_{j}+d_{j}-1$, $\sigma_{11, j}=0$, and where $\mathrm{ff}=\mathrm{ff}_{1} \cup \mathrm{ff}_{2}$.

Proof. To separate out the contributions from the near-diagonal parts of each factor, we use standard results concerning the symbol calculus with spectral parameter (cf. [26]). Write $K_{j}=A_{j}+B_{j}$ where $A_{j}$ contains the full diagonal singularity, is supported near the lifted diagonal in $\left(M_{j}\right)_{\mathbb{K}_{j} \Theta}^{2}$, and is smooth across the front face of this double space, while $B_{j} \in \mathscr{A}_{\mathrm{ff}}^{\sigma_{j}}\left(\left(M_{j}\right)_{\mathbb{K}_{j} \Theta}^{2}\right), \sigma_{j}=\left(\left(n_{j}+d_{j}-1\right) / 2+\delta_{0}^{\mathbb{K}_{j}},\left(n_{j}+d_{j}-1\right) / 2+\delta_{0}^{\mathbb{K}_{j}}\right)$.

We first analyze the integral of $A_{1}(i \mu) A_{2}(-i \mu)$. Recall from [26] that the norm of $A_{j}$ as a map on any fixed weighted Sobolev or weighted Hölder space decays like $1 /|\mu|$. In that source, this result is only stated in the compact case, but applies here equally well because of the support properties of $A_{j}$. In any case, this shows that this integral converges 
in the operator norm on any one of these spaces. It is also clear that its Schwartz kernel is supported in a neighbourhood of $\operatorname{diag}_{\mathrm{ph}}$ which intersects the front faces of $M_{\mathrm{ph}}^{2}$ but not the side faces. This distribution may be estimated directly (just as for the analogous computation on a product of two compact manifolds) using the oscillatory integral representations in the conormal bundle of $\operatorname{diag}_{\mathrm{ph}}$, and from this we see that it is smooth up to the front faces and has a pseudodifferential singularity of order -2 along the diagonal, as required.

Using similar arguments, we deduce that the integrals of $A_{1}(i \mu) B_{2}(-i \mu)$ and $A_{2}(i \mu) B_{1}(-i \mu)$ are smooth on the interior of $M_{\mathrm{ph}}^{2}$. By taking any number of derivatives with respect to $b$-vector fields on the $B$ factor, we obtain the appropriate conormal estimates too.

As for the integral of $B_{1}(i \mu) B_{2}(-i \mu)$, divide the contour $\Gamma_{0}$ into a compact portion, where $|\mu| \leq \mu_{0}$, and its remaining noncompact ends $|\mu| \geq \mu_{0}$. The conormal estimates on $B_{j}$ hold locally uniformly in $\mu$, so in particular

$$
\int_{|\mu| \leq \mu_{0}} B_{1}\left(z_{1}, z_{1}^{\prime}, i \mu\right) B_{2}\left(z_{2}, z_{2}^{\prime},-i \mu\right) d \mu \in \mathscr{A}_{B_{11}}^{\sigma}\left(M_{\mathrm{ph}}^{2}\right) .
$$

On the other hand, using the exponential bounds on $K_{j}$ from Proposition 17 we immediately deduce that the integral over $|\mu|>\mu_{0}$ satisfies the same $\mathscr{C}^{0}$ bound. The estimates for higher tangential derivatives required to check conormality are obtained in exactly the same way, using the corresponding pointwise bounds for the higher tangential derivatives on each of the two factors.

This completes the estimation of the Schwartz kernel $G$.

\section{Modifications when either factor has negative point spectrum}

If inf $\operatorname{spec}\left(L_{1}\right)$ or inf $\operatorname{spec}\left(L_{2}\right)$ is negative, then (7.2) needs to be altered slightly. To understand this, begin by noting that (7.1) remains valid when $\lambda=i \epsilon$ and the contour separates the sets $i \epsilon-\operatorname{spec}\left(L_{1}\right)$ and $\operatorname{spec}\left(L_{2}\right)$ in two different half-planes. In fact, we take the contour to be the union of the vertical rays $[2 i \epsilon, i \infty),(-i \infty,-i \epsilon]$ and two long thin half-ellipses, one in the first quadrant with minor axis connecting $\frac{1}{2} i \epsilon$ and $2 i \epsilon$, and the other in the third quadrant, with minor axis connecting $-\frac{1}{2} i \epsilon$ and $\frac{1}{2} i \epsilon$. Now let $\epsilon \searrow 0$; we arrive at the formula that $\left(L_{1}+L_{2}\right)^{-1}$ is equal to the sum

$$
-\frac{1}{2 \pi i} \int_{-i \infty}^{i \infty} R_{1}(-\mu) R_{2}(\mu) d \mu+\sum_{\mu_{j}^{(1)}<0} P_{1}\left(-\mu_{j}^{(1)}\right) R_{2}\left(\mu_{j}^{(1)}\right)+\sum_{\mu_{j}^{(2)}<0} R_{1}\left(-\mu_{j}^{(2)}\right) P_{2}\left(\mu_{j}^{(2)}\right) .
$$

Here $\mu_{j}^{(i)}$ are the (negative) eigenvalues of $L_{i}$, and $P_{i}\left(\mu_{j}^{(i)}\right)$ is the orthogonal projection onto the corresponding eigenspace in the $i^{\text {th }}$ factor.

The main result in the last subsection, that the Schwartz kernel of $\left(L^{g}\right)^{-1}$ is conormal on $M_{\mathrm{ph}}^{2}$, clearly remains valid. The analysis of the main term, which is the first summand in this expression, is exactly the same as before. The remaining terms are much simpler to analyze since each is a simple tensor product of one term which is polyhomogeneous on one factor and a finite rank polyhomogeneous term on the other factor. 


\subsection{Function spaces and mapping properties}

We now introduce the natural geometric Hölder spaces on the product space $M$ with respect to a weakly product hyperbolic metric and describe the mapping properties for the types of pseudodifferential operators encountered in the last subsection. These mapping properties are needed to conclude that $L^{g}$ is actually invertible when $g$ is an approximate Einstein metric sufficiently close to a product. Indeed, the arguments in the rest of this paper are complicated by the fact that if $\mathbb{K}_{1}=\mathbb{H}$, then the Hölder spaces corresponding to two different weakly product hyperbolic metrics $g$ and $g^{\prime}$ are not quasi-isometric to one another, unless the corresponding distributions $\mathscr{D}_{1}$ and $\mathscr{D}_{1}^{\prime}$ on $\partial M_{1} \times M_{2}$ are diffeomorphically equivalent. This failure of the Darboux lemma is the reason we must study the inverse of $L^{g}$ for $g$ near to but not exactly equal to a product metric.

Closely following $\S 3$, the class of geometric Hölder spaces $x_{1}^{\nu_{1}} x_{2}^{\nu_{2}} \Lambda_{\mathrm{ph}}^{k, \alpha}(M)$ is defined relative to differentiations by locally finite combinations of smooth multiples of elements of $\mathscr{V}_{\mathbb{K}_{1} \Theta}\left(M_{1}\right)+\mathscr{V}_{\mathbb{K}_{2} \Theta}\left(M_{2}\right)$, and by Hölder difference quotients adapted to the distance relative to the weakly product hyperbolic metric $g$.

Proposition 39. Let $H$ have Schwartz kernel in $\mathscr{A}_{\mathrm{ff}}^{\sigma}\left(M_{\mathrm{ph}}^{2}\right.$, $\left.\operatorname{diag}_{\mathrm{ph}}\right)$, where $\sigma$ is the usual weight family with $\sigma_{11, j}=0$ and all other $\sigma_{p q, j}=\left(n_{j}+d_{j}-1\right) / 2+\delta_{0}^{\mathbb{K}_{j}}$, and with pseudodifferential order $-r<0$ along $\operatorname{diag}_{\mathrm{ph}}$. Then, provided $0<v_{j}<\left(n_{j}+d_{j}-1\right) / 2+\delta_{0}^{\mathbb{K}_{j}}$, $j=1,2$, the map

$$
H: x_{1}^{\nu_{1}} x_{2}^{\nu_{2}} \Lambda_{\mathrm{ph}}^{k, \alpha}(M) \rightarrow x_{1}^{\nu_{1}} x_{2}^{\nu_{2}} \Lambda_{\mathrm{ph}}^{k+r, \alpha}(M)
$$

is bounded. If $Q$ has Schwartz kernel in $\mathscr{A}^{\tilde{\sigma}}\left(M_{\mathrm{ph}}^{2}\right)$ (hence no interior singularities), where $\tilde{\sigma}_{11, j}>0$ but all other weights are the same as for $\sigma$, then $Q$ is a compact mapping between these same spaces.

Proof. As in Proposition 10, decompose $H=H^{\prime}+H^{\prime \prime}$. The boundedness of $H^{\prime}$ follows from the classical interior boundedness of pseudodifferential operators on Hölder spaces and the fact that the Schwartz kernel of $H^{\prime}$ is supported in a tube of finite radius around the diagonal of $M_{1} \times M_{2}$.

The proof of the weighted $\mathscr{C}^{0}$ bound for $H^{\prime \prime} f$ when $f$ is in the weighted ph-Hölder space proceeds by a direct and elementary estimation of this integral. Any higher derivative of $H^{\prime \prime} u$ with respect to a $\mathbb{K}_{1} \Theta$ vector field on the first factor or a $\mathbb{K}_{2} \Theta$ vector field on the second factor, or an iterated combination of such vector fields, is handled by noting that any of these vector fields applied to $H^{\prime \prime}$ gives a Schwartz kernel of exactly the same form, with the same orders of vanishing at all the side faces.

Finally, to prove that $Q$ is a compact operator, by Proposition 10 again, the fact that it has order $-\infty$ and vanishes to some positive order at the front faces gives

$$
Q: x_{1}^{\nu_{1}} x_{2}^{\nu_{2}} \Lambda_{\mathrm{ph}}^{s_{1}, \alpha}(M) \rightarrow x_{1}^{\nu_{1}+\varepsilon} x_{2}^{\nu_{2}+\varepsilon} \Lambda_{\mathrm{ph}}^{s_{2}, \alpha}(M)
$$

for any $s_{1}, s_{2} \in \mathbb{N}$. This range space on the right includes compactly into the domain space on the left (provided $s_{2}>s_{1}$ ), using the Arzelà-Ascoli theorem.

In the course of the parametrix construction, we shall need a slightly refined version of this result which allows us to conclude that a solution $u$ to $L^{g} u=f$ has as much tangential 
regularity as $f$ does, and in particular that $u$ is conormal if the same is true of $f$. In order to measure tangential regularity, we introduce a class of hybrid spaces which allow some of the $\mathscr{V}_{\mathbb{K}_{1} \Theta}\left(M_{1}\right)+\mathscr{V}_{\mathbb{K}_{2} \Theta}\left(M_{2}\right)$ derivatives to be replaced by $\mathscr{V}_{b}\left(M_{1} \times M_{2}\right)$ derivatives. For want of a better name, we denote such a space by $x_{1}^{\nu_{1}} x_{2}^{\nu_{2}} \Lambda_{\mathrm{ph}-b}^{k, \ell, \alpha}(M)$ for any $0 \leq \ell \leq k$; it consists of functions $u \in x_{1}^{\nu_{1}} x_{2}^{\nu_{2}} \Lambda_{\mathrm{ph}}^{k, \alpha}(M)$ such that $V_{1} \ldots V_{j} u \in x_{1}^{\nu_{1}} x_{2}^{\nu_{2}} \Lambda_{\mathrm{ph}}^{k-j, \alpha}(M)$ for any $b$-vector fields $V_{1}, \ldots, V_{j} \in \mathscr{V}_{b}(M)$ and any $j \leq \ell$. The index $\ell$ denotes the degree of full tangential regularity and

$$
\bigcap_{k \geq \ell \geq 0} x_{1}^{\nu_{1}} x_{2}^{\nu_{2}} \Lambda_{\mathrm{ph}-b}^{k, \ell, \alpha}(M)=\mathscr{A}^{\nu_{1}, \nu_{2}}(M),
$$

the space of conormal functions on $M$ vanishing like $x_{1}^{\nu_{1}}$ at $\partial M_{1} \times M_{2}$ and $x_{2}^{\nu_{2}}$ at $M_{1} \times \partial M_{2}$.

Corollary 40. Let $H$ and $v_{1}, v_{2}$ be exactly the same as in Proposition 39. Then for any $k \geq 0$ and $0 \leq \ell \leq k$,

$$
H: x_{1}^{\nu_{1}} x_{2}^{\nu_{2}} \Lambda_{\mathrm{ph}-b}^{k, \ell, \alpha}(M) \rightarrow x_{1}^{\nu_{1}} x_{2}^{\nu_{2}} \Lambda_{\mathrm{ph}-b}^{k+r, \ell, \alpha}(M)
$$

is bounded.

Proof. The key idea is a commutation relation: if $V$ is any $b$-vector field on $M$ and $V_{L}$ and $V_{R}$ denote its lifts to $M^{2}$ from the left and right factors, respectively, then the Schwartz kernel of the composition $\left(V_{L}+V_{R}\right) H$ has exactly the same regularity properties and index sets on $M_{\mathrm{ph}}^{2}$ as $H$ itself. This is established by straightforward calculation. The subtlety concerns its behaviour near the two front faces since $V_{L} H$ and $V_{R} H$ individually blow up to first order there, but these leading singular terms cancel and the sum is again bounded here. This follows from the cancellation for the analogous computation on $\left(M_{j}\right)_{\mathbb{K}_{j} \Theta}^{2}$, which can be found in $\left[18\right.$, Proposition 3.30] when $\mathbb{K}_{j}=\mathbb{R}$, and can be proved in exactly the same way in the other cases.

\subsection{Near-product hyperbolic metrics and the parametrix construction}

If $g=g_{1}+g_{2}$ is a nondegenerate product hyperbolic metric, then we proved in $\S 7.1$ that the Schwartz kernel of the $L^{2}$ inverse of $L^{g}$ is conormal on the product hyperbolic double space. Consequently, by the results of $\S 7.2$, this inverse is bounded on certain weighted Hölder spaces, hence $L^{g}$ is also invertible on those spaces. Our goal is to establish that $L^{g}$ is invertible on these weighted Hölder spaces when $g$ is strongly asymptotically Einstein and sufficiently close to a product metric. For this it suffices to establish a similar structure theorem for a parametrix $H$ for $\left(L^{g}\right)^{-1}$; more precisely, we construct $H$ and show that it has Schwartz kernel in $\Psi_{\mathrm{ph}}^{-2}(M)+\mathscr{A}_{\mathrm{ff}}^{\sigma}\left(M_{\mathrm{ph}}^{2}\right)$, where $\sigma$ is the index family from Proposition 39, which implies that it is bounded and the error terms $L^{g} H-I$ and $H L^{g}-I$ have small norm, and hence finally that $L^{g}$ is invertible on these same spaces.

The parametrix construction is much the same as in the AKKH case. The Schwartz kernels of general ph pseudodifferential operators are by definition distributions on $M_{\mathrm{ph}}^{2}$ 
which are conormal at the boundaries and polyhomogeneous at the diagonal. There is a small calculus of operators with Schwartz kernels supported near the lifted diagonal, and a large calculus which also includes operators with Schwartz kernels conormal up to all faces, and with positive vanishing order at the front faces. The corresponding decomposition of the parametrix $H$ is written $H^{\prime}+H^{\prime \prime}$.

Let $g$ be a weakly asymptotically product hyperbolic metric on $M$. The initial approximation $H^{\prime}$ to $\left(L^{g}\right)^{-1}$ is obtained via the standard elliptic parametrix construction using the symbol calculus on the conormal bundle of $\operatorname{diag}_{\mathrm{ph}} \subset M_{\mathrm{ph}}^{2}$. This is done exactly as in the AKKH setting, and simply uses the uniform invertibility of the ph-symbol of $L^{g}$ on $M_{\mathrm{ph}}^{2}$ up to the two front faces (this is valid for any weakly asymptotically product hyperbolic metric). If $g$ is only polyhomogeneous at the boundaries of $M$, then $H^{\prime} \in \mathscr{A}_{\mathrm{ff}}^{\tau}\left(M_{\mathrm{ph}}^{2}, \operatorname{diag}_{\mathrm{ph}}\right)$, where $\tau_{10, j}=\tau_{01, j}=\infty$ and $\tau_{11, j}=0, j=1,2$.

Set $I-L^{g} H^{\prime}=Q_{0}$; this is an element of $\mathscr{A}_{\mathrm{ff}}^{\tau}\left(M_{\mathrm{ph}}^{2}\right)$. The correction term $H^{\prime \prime}$ is chosen so that $L^{g}\left(H^{\prime}+H^{\prime \prime}\right)=I-Q_{1}$, or equivalently, $L^{g} H^{\prime \prime}=Q_{0}-Q_{1}$, where $H^{\prime \prime} \in \mathscr{A}_{\mathrm{ff}}^{\sigma}\left(M_{\mathrm{ph}}^{2}, \operatorname{diag}_{\mathrm{ph}}\right)$ and $Q_{1} \in \mathscr{A}^{\tilde{\sigma}}\left(M_{\mathrm{ph}}^{2}\right)$; here $\sigma_{11, j}=0, \tilde{\sigma}_{11, j}>0$, and all other $\sigma_{p q, j}=\tilde{\sigma}_{p q, j}=n_{j}+d_{j}-1$ for $p q \neq 11$. The Schwartz kernel of $Q_{1}$ vanishes to some positive order at the front faces so it is compact on weighted Hölder spaces.

To determine $H^{\prime \prime}$, restrict the equation $L^{g} H^{\prime \prime}=Q_{0}-Q_{1}$ to each of the two front faces. Set

$$
N_{j}\left(L^{g}\right)=\left.L^{g}\right|_{\mathrm{ff}_{j}}, \quad N_{j}\left(Q_{0}\right)=\left.Q_{0}\right|_{\mathrm{ff}_{j}}, \quad j=1,2 .
$$

We must first solve the two equations $N_{j}\left(L^{g}\right) H_{j}^{\prime \prime}=N_{j}\left(Q_{0}\right)$ and then choose some $H^{\prime \prime}$ which has normal operators at $\mathrm{ff}_{j}$ equal to these $H_{j}^{\prime \prime}$. Note that $N_{j}\left(Q_{0}\right)$ is smooth on the interior of $\mathrm{ff}_{j}$, vanishes to infinite order at the intersection with the side faces, and is smooth up to the other front face, or at least, the dependence in this direction is exactly as regular as the metric $g$ near the corner.

We begin by analyzing the structure of $N_{j}\left(L^{g}\right)$ when $g$ is weakly product hyperbolic. Fixing $j=2$ to be definite, the front face $\mathrm{ff}_{2}$ is a product $\left(M_{1}\right)_{\mathbb{K}_{1} \Theta}^{2} \times \mathrm{ff}\left(M_{2}\right)$. The second factor, which is the front face of $\left(M_{2}\right)_{\mathbb{K}_{2} \Theta}^{2}$, is a fibration with base space the diagonal of $\left(\partial M_{2}\right)^{2}$ and each fibre identified with the hyperbolic space $\mathbb{K}_{2} H^{m_{2}}$. As we explain below, the identification is not completely natural, however, when $\mathbb{K}_{2}=\mathbb{R}$ or $\mathbb{C}$, but is natural in the remaining two cases $\mathbb{K}_{2}=\mathbb{H}$ or $\mathbb{O}$. The lift $L^{g}$ from the left factor of $M$ to $M_{\mathrm{ph}}^{2}$ acts on the 'left factor' of $M_{j}$ in each $\mathbb{K}_{j} \Theta$ double space. It acts tangentially to all boundary faces on $M_{\mathrm{ph}}^{2}$, and in particular, its restriction to $\mathrm{ff}_{2}$ is a sum of products of derivatives of two types: some which act on the left factor of $M_{1}$ in $\left(M_{1}\right)_{\mathbb{K}_{1} \Theta}^{2}$ and others which act on the $\mathbb{K}_{2} H^{m_{2}}$ fibres of $\mathrm{ff}\left(M_{2}\right)$. A priori, there could be 'mixed' terms of second order, which differentiate once in each of these directions. On the other hand, the dependence on all other variables - namely, in the right factor of $M_{1}$ and in the diagonal of $\left(\partial M_{2}\right)^{2}$ (i.e. the base of the fibration of $\mathrm{ff}\left(M_{2}\right)$ ) - is purely parametric.

Definition 41. We say that a weakly asymptotically product hyperbolic metric $g$ on $M_{1} \times M_{2}$ is near-product hyperbolic if the normal operators $N_{1}\left(L^{g}\right), N_{2}\left(L^{g}\right)$ have the 
following special form:

$$
N_{1}\left(L^{g}\right)=L_{1,1}+L_{1,2} \quad \text { and } \quad N_{2}\left(L^{g}\right)=L_{2,1}+L_{2,2},
$$

where, for example, in the second decomposition $L_{2,1}$ is an operator acting on the left factor of $M_{1}$ (with coefficients depending parametrically on the right factor of $M_{1}$ and the diagonal of $\left.\left(\partial M_{2}\right)^{2}\right)$ and $L_{2,2}$ is an operator acting on the front face $\operatorname{ff}\left(M_{2}\right)$.

Let us investigate when this condition holds. Focussing again on $N_{2}\left(L^{g}\right)$, use interior coordinates $z_{1}$ on $M_{1}$ and $z_{2}=\left(x_{2}, y_{2}\right)$ near the boundary of $M_{2}$. Then, near $x_{2}=0, L^{g}$ is a sum of products of various of the $\partial_{z_{1}}$, and of $x_{2} \partial_{x_{2}}, x_{2} Y$ and $x_{2}^{2} Z$, where $\left(Y_{1}, \ldots, Y_{d_{2}\left(m_{2}-1\right)}\right)$ and $\left(Z_{1}, \ldots, Z_{d_{2}-1}\right)$ are bases of sections of $\mathscr{D}_{2}$ and its complement, respectively, in $T X_{2}$. Now lift to $M_{\mathrm{ph}}^{2}$ and near $\mathrm{ff}_{2}$ replace $\left(z_{2}, \tilde{z}_{2}\right)$ with projective coordinates; thus $y_{2}=\tilde{y}_{2}+\mathscr{O}\left(\tilde{x}_{2}\right)$ and at $\tilde{x}_{2}=0$, the derivatives with respect to $x_{2}, Y_{i}$ and $Z_{j}$ take the form $s \partial_{s}, s Y_{i}^{0}, s^{2} Z_{j}^{0}$, where $s=x_{2} / \tilde{x}_{2}$ and the $Y_{i}^{0}$ and $Z_{j}^{0}$ are a basis of left-invariant forms on $\mathbb{K}_{2} \mathrm{Heis}^{m_{2}-1}$, all as explained in $\S 3$. In particular, these vector fields are tangent to the fibres of $\mathrm{ff}\left(M_{2}\right)$. This restriction to $\tilde{x}_{2}$ defines $N_{2}(L)$, so we see that $g$ is near-product hyperbolic provided that there are no cross-terms, i.e.

$$
N_{2}\left(L^{g}\right)=\sum a_{\alpha}^{(1)}\left(z_{1}, \tilde{y}_{2}\right) \partial_{z_{1}}^{\alpha}+\sum a_{j, \beta, \gamma}^{(2)}\left(z_{1}, \tilde{y}_{2}\right)\left(s \partial_{s}\right)^{j}\left(s Y^{0}\right)^{\beta}\left(s^{2} Z^{0}\right)^{\gamma},
$$

and in addition, as noted before, the endomorphism coefficients have no off-diagonal components with respect to the splitting into the two factors. Note that the coefficients $a_{\alpha}^{(1)}$ depend on $\left(z_{1}, \tilde{y}_{2}\right)$, but not on the 'active' variables in the second factor. On the other hand, the coefficients $a_{j, \beta, \gamma}^{(2)}$ potentially depend on all active variables on $\mathrm{ff}_{2}$. (To be clear, the nonactive variables are $\tilde{z}_{1}$ and $\tilde{y}_{2}$; these parametrize the base of the fibration of $\mathrm{ff}_{2}$.)

The fibres of $\operatorname{ff}\left(M_{2}\right)$ are identified with $\mathbb{K}_{2} H^{m_{2}}$, and it is clear that the second summand above, which in our previous notation is $L_{2,2}$, is left-invariant on this hyperbolic space. By the naturality of the geometric operators we are considering, we expect that $L_{2,2}$ is simply the linearized gauged Einstein operator or the rough Laplacian on $\mathbb{K}_{2} H^{m_{2}}$. This is indeed true, but only after a choice of basis of left-invariant vector fields (adapted to the boundary contact structure) on this space, and it is an important but not immediately obvious fact that this choice may depend smoothly on $z_{1}$. More clearly, the identification of $\mathrm{ff}\left(M_{2}\right)$ with $\mathbb{K}_{2} H^{m_{2}}$ is determined only up to a linear isomorphism which preserves the model contact structure on $\mathbb{K}_{2} \mathrm{Heis}^{m_{2}-1}$. When $\mathbb{K}_{2}=\mathbb{H}$ or $\mathbb{O}$, this determines the isomorphism completely since in these cases the contact structure determines the metric fully. However, when $\mathbb{K}_{2}=\mathbb{R}$ or $\mathbb{C}$, there are different linear identifications which are isometric, but not identical. For example, in the real case, this corresponds to the fact that any metric of the form

$$
\frac{d x^{2}+\sum_{i, j=1}^{n-1} a_{i j} d y^{i} d y_{j}}{x^{2}},
$$

where $\left(a_{i j}\right)$ is a constant positive definite symmetric matrix, is isometric to real hyperbolic space, but the isometry is defined in terms of the coefficients $a_{i j}$. In our setting, when $\mathbb{K}_{2}=\mathbb{R}$, these coefficients will depend smoothly on $z_{1}$, and for metrics which are 
very close to being a product globally, they will be very close to constant. Thus altogether, when $\mathbb{K}_{2}=\mathbb{R}$ or $\mathbb{C}, L_{2,2}$ must be thought of as a family of generalized Laplacians on $\mathbb{K}_{2} H^{m_{2}}$ which depend smoothly on the variable $z_{1}$. Hence $N_{2}\left(L^{g}\right)$ is not a product operator as studied in $\$ 7.1$. The fact which saves us in the setting of this paper is that since $g$ is a small perturbation of a product metric, the deviation of $L_{2,2}$ from an operator which does not depend on $z_{1}$ is very small.

Lemma 42. Let $g$ be a strongly asymptotically Einstein metric, as in Definition 37. Then $g$ is near-product hyperbolic.

Proof. By the global rigidity assumption for the conformal infinity data, we are fixing the product structure on each boundary hypersurface of $M_{1} \times M_{2}$. Consider the construction of $g$ at $M_{1} \times \partial M_{2}$. The preliminary extensions of the conformal infinity data $\left(\gamma_{1}, \eta_{1}\right)$ and $\gamma_{2} / x_{2}^{2}+\eta_{2} / x_{2}^{4}$, regarded as metrics on the bundle $T M_{1} \oplus{ }^{\mathbb{K}_{2} \Theta} T_{\partial M_{2}} M_{2}$ over this face, make these subbundles orthogonal to one another. The correction terms $h$ and $\phi$ preserve this orthogonality. The lemma follows directly from this.

Having established this, we now proceed with the parametrix construction by solving the two normal equations, say the one on $\mathrm{ff}_{2}$ again to be definite. The equation $N_{2}\left(L^{g}\right) N_{2}\left(H^{\prime \prime}\right)$ $=N_{2}\left(Q_{0}\right)$ depends parametrically on the right factor of $M_{1}$ in $\left(M_{1}\right)_{\mathbb{K}_{1} \Theta}^{2}$ and on $\partial M_{2}$; thus there is a separate equation to solve for each $\left(\tilde{x}_{1}, \tilde{y}_{1}, \tilde{y}_{2}\right)$. When $\tilde{x}_{1}>0$, this is an equation on $M_{1} \times \mathrm{ff}\left(M_{2}\right)$, and $N_{2}\left(Q_{0}\right)$ is smooth and compactly supported. When $\tilde{x}_{1}=0$, there are two cases: one is for the induced equation on the product of the right face of $\left(M_{1}\right)_{\mathbb{K}_{1} \Theta}^{2}$ (which is a copy of $M_{1}$ blown up at the boundary point $\left.\tilde{y}_{1}\right)$ with $\mathrm{ff}\left(M_{2}\right)$, and the other is for the induced equation on $\mathrm{ff}\left(M_{1}\right) \times \mathrm{ff}\left(M_{2}\right)$. Noting that $N_{2}\left(Q_{0}\right)$ vanishes identically on the space appearing in the middle case here, we set $N_{2}\left(H^{\prime \prime}\right)=0$ there, so we are left to understand this equation on $M_{1} \times \mathrm{ff}\left(M_{2}\right)$ and on $\mathrm{ff}\left(M_{1}\right) \times \mathrm{ff}\left(M_{2}\right)$, with $N_{2}\left(Q_{0}\right) \in \mathscr{C}_{0}^{\infty}$ in either case.

The latter case, on the product of the front faces, is the more elementary one since at this face, $N_{2}\left(L^{g}\right)$ is always an exact product operator, hence can be analyzed directly using the results of $\S 7.1$. More specifically, the restriction of $N_{2}\left(L^{g}\right)$ to this corner is the sum of model generalized Laplacians on $\mathbb{K}_{1} H^{m_{1}} \times \mathbb{K}_{2} H^{m_{2}}$; since it is of product type, the results of $\S 7.1$ show that $N_{2}\left(H^{\prime \prime}\right)=N_{2}\left(L^{g}\right)^{-1} N_{2}\left(Q_{0}\right)$ is conormal on $\mathrm{ff}\left(M_{1}\right) \times$ $\mathrm{ff}\left(M_{2}\right)$. Of course, $N_{2}\left(Q_{0}\right)$ depends smoothly on the parameters $\left(\tilde{y}_{1}, \tilde{y}_{2}\right)$, and the solution depends smoothly on these parameters.

The same analysis can be applied directly for the other case, i.e. for the equation on $M_{1} \times \operatorname{ff}\left(M_{2}\right)$, when $\mathbb{K}_{2}=\mathbb{H}$ or $\mathbb{O}$, since in these cases $N_{2}\left(L^{g}\right)$ is still of product type for each $\left(\tilde{z}_{1}, \tilde{y}_{2}\right)$. Taking into account the smooth dependence on these parameters and the conormal structure of $N_{2}\left(L^{g}\right)^{-1}$, we see that $N_{2}\left(H^{\prime \prime}\right)$ is conormal on $\mathrm{ff}_{2}$, at least away from the corner $\mathrm{ff}_{1} \cap \mathrm{ff}_{2}$. We discuss the behaviour at this corner momentarily.

It remains to understand this family of problems on $M_{1} \times f f\left(M_{2}\right)$ when $\mathbb{K}_{2}=\mathbb{R}$ or $\mathbb{C}$. As we have described, $N_{2}\left(L^{g}\right)$ is no longer necessarily of product type; instead, when the near-product hyperbolic metric $g$ is sufficiently close to a product (which is certainly the case for the strongly asymptotically Einstein metrics we have constructed), it can be decomposed as a sum $A+B$ where $A$ is of product type and $B$ is a second order 
operator with small coefficients. The product analysis of $\$ 7.1$ shows that $A^{-1}$ exists and that its Schwartz kernel has conormal structure. Now write $(A+B) A^{-1}=I+B A^{-1}$. We now invoke the mapping properties from Corollary 40 to see that $B A^{-1}$ is bounded and has very small norm on any one of the spaces $x_{1}^{\nu_{1}} s_{2}^{\nu_{2}} \Lambda_{\mathrm{ph}-b}^{k, \ell, \alpha}\left(M_{1} \times \mathrm{ff}\left(M_{2}\right)\right)$, hence $I+B A^{-1}$ is invertible here. This implies that if $f \in \mathscr{C}_{0}^{\infty}$, then the solution $u$ to the equation $(A+B) u=f$ lies in the intersection over all $k$ and $\ell$ of these hybrid weighted Hölder spaces (for fixed weights $v_{1}, v_{2}$ ), hence is conormal up to the boundaries, at least away from $\mathrm{ff}_{1} \cap \mathrm{ff}_{2}$. In a similar way, we can find the solution $N_{1}\left(H^{\prime \prime}\right)$ on the interior of $\mathrm{ff}_{1}$ which is conormal up to the boundaries, at least away from $\mathrm{ff}_{1} \cap \mathrm{ff}_{2}$.

The final step is to analyze how these solutions fit together at the corner $\mathrm{ff}_{1} \cap \mathrm{ff}_{2}$. For this, first note that there is an obvious compatibility between the two normal operators at this corner:

$$
\left.N_{1}\left(L^{g}\right)\right|_{\mathrm{ff}_{1} \cap \mathrm{ff}_{2}}=\left.N_{2}\left(L^{g}\right)\right|_{\mathrm{ff}_{1} \cap \mathrm{ff}_{2}} .
$$

By uniqueness of the inverse, we see that

$$
\begin{aligned}
\left.N_{1}\left(H^{\prime \prime}\right)\right|_{\mathrm{ff}_{1} \cap \mathrm{ff}_{2}}: & =\left.\left.N_{1}\left(L^{g}\right)\right|_{\mathrm{ff}_{1} \cap \mathrm{ff}_{2}} ^{-1} N_{1}\left(Q_{0}\right)\right|_{\mathrm{ff}_{1} \cap \mathrm{ff}_{2}} \\
& =\left.\left.N_{2}\left(L^{g}\right)\right|_{\mathrm{ff}_{1} \cap \mathrm{ff}_{2}} ^{-1} N_{2}\left(Q_{0}\right)\right|_{\mathrm{ff}_{1} \cap \mathrm{ff}_{2}}:=\left.N_{2}\left(H^{\prime \prime}\right)\right|_{\mathrm{ff}_{1} \cap \mathrm{ff}_{2}} .
\end{aligned}
$$

The fact that $N_{j}\left(H^{\prime \prime}\right)$ is conormal up to this corner is a consequence of the fact that the parametric dependence of $N_{j}\left(Q_{0}\right)$ is conormal, and the inverses preserve conormality in these parameters.

Now extend the two functions $N_{j}\left(H^{\prime \prime}\right)$ on $\mathrm{ff}_{1} \cup \mathrm{ff}_{2}$ to a Schwartz kernel $H^{\prime \prime} \in$ $\mathscr{A}_{\mathrm{ff}}^{\sigma}\left(M_{\mathrm{ph}}^{2}\right)$ which restricts to $N_{j}\left(H_{2}\right)$ at $\mathrm{ff}_{j}$.

We have now constructed $H=H^{\prime}+H^{\prime \prime}$ which satisfies $L^{g} H=I-Q_{1}, Q_{1} \in$ $\mathscr{A}^{\tilde{\sigma}}\left(M_{\mathrm{ph}}^{2}\right)$. By Proposition 39, $Q_{1}$ is a compact operator on the weighted ph Hölder spaces. A left parametrix is obtained by taking adjoints. This completes the parametrix construction when $g$ is near-product hyperbolic and polyhomogeneous, and proves that $L^{g}$ is Fredholm.

As explained earlier, we can certainly restrict to studying polyhomogeneous metrics with smooth conformal infinity data but we comment briefly on how to extend this proof to the case where $g$ is the sum of a polyhomogeneous A $\mathbb{K} H$ metric and a perturbation term $k \in x_{1}^{\nu_{1}} x_{2}^{\nu_{2}} \Lambda_{\mathbb{K} \Theta}^{2, \alpha}$, where $0<v_{j}<n_{j}+d_{j}-1$. (Note that this is the regularity for the strongly asymptotically Einstein metrics.)

The symbol calculus step goes through immediately for symbols with this regularity. Indeed, the perturbation term $k$ appears only in the parametric dependence along the diagonal and does not occur in the leading terms near the front faces, so $H^{\prime}$ decomposes as a principal polyhomogeneous term and another, vanishing to some positive order at these faces but which is only bounded in Hölder norm. The restriction to the front faces of the error term $Q=I-L H^{\prime}$ does not depend on these lower order terms, and the normal operators $N_{j}(L)$ are independent of them too, except as above in their dependence on parameters, which means that the second step carries through exactly as before. 
We have proved

Proposition 43. Let $g$ be a strongly asymptotically Einstein metric as constructed in §6.2. Then there is a parametrix $H=H^{\prime}+H^{\prime \prime}$ for $L^{g}$, with $H^{\prime} \in \mathscr{A}_{\mathrm{ff}}^{\sigma}\left(M_{\mathrm{ph}}^{2}\right.$, $\left.\operatorname{diag}_{\mathrm{ph}}\right)$ and $H^{\prime \prime} \in \mathscr{A}_{\mathrm{ff}}^{\sigma}\left(M_{\mathrm{ph}}^{2}\right)$. The error term $Q_{1}=I-L\left(H^{\prime}+H^{\prime \prime}\right)$ is in $\mathscr{A}_{\mathrm{ff}}^{\tilde{\sigma}}\left(M_{\mathrm{ph}}^{2}\right)$.

Theorem 44. Let $g$ be a near-product hyperbolic metric which is sufficiently close to a nondegenerate product hyperbolic metric $g_{0}$ on $M_{1} \times M_{2}$. Then

$$
L^{g}: x_{1}^{\nu_{1}} x_{2}^{\nu_{2}} \Lambda_{\mathrm{ph}}^{2, \alpha}\left(M, \operatorname{Sym}^{2}\left(T^{*} M\right)\right) \rightarrow x_{1}^{\nu_{1}} x_{2}^{\nu_{2}} \Lambda_{\mathrm{ph}}^{0, \alpha}\left(M, \operatorname{Sym}^{2}\left(T^{*} M\right)\right)
$$

is an isomorphism.

Proof. If $g$ is any near-product hyperbolic metric, then we have constructed a parametrix $H^{g}$ for $L^{g}$ so that $L^{g} H^{g}=H^{g} L^{g}=I-Q^{g}$. We have also proved that $H^{g}$ is a bounded operator between the space on the right and the space on the left above, and that $Q^{g}$ is compact between these same spaces. This proves that the map $L^{g}$ is Fredholm.

The construction of $H^{g}$ depends continuously on the metric (with respect to some sufficiently strong topology). Since $Q^{g}=0$ when $g=g_{0}$, we can make the norm of $Q^{g}$ as small as desired when $g$ is sufficiently close to $g_{0}$, which implies that $L^{g}$ is then invertible.

\section{Solving for the Einstein metric}

The remainder of the proof of the Main Theorem proceeds very much as in the analogous arguments in $\$ 4$ and $\S 6$.

Let $\left(M=M_{1} \times M_{2}, g=g_{1}+g_{2}\right)$ be a product of AKH Einstein metrics. Let $L^{g}$ and $L^{g_{i}}$ be the linearized gauged Einstein operators for $g$ and the two component metrics $g_{i}$. We assume that

$$
0 \notin \operatorname{spec}\left(L^{g}\right) \cup \operatorname{spec}\left(L^{g_{1}}\right) \cup \operatorname{spec}\left(L^{g_{2}}\right),
$$

and that at least one of $\mathbb{K}_{1}$ or $\mathbb{K}_{2}$ is not equal to $\mathbb{H}$ or $\mathbb{O}$. Denote by $\mathfrak{c}$ the $\left(G_{1} \times G_{2}\right)$ conformal infinity data on $X=X_{1} \times X_{2}=\partial M_{1} \times \partial M_{2}$.

Theorem 45. Under all these conditions, let $\mathfrak{c}^{\prime}$ be any globally integrable $\left(G_{1} \times G_{2}\right)$ conformal infinity data on $X$ which is $\mathscr{C}^{\infty}$ and sufficiently close (in $\mathscr{C}^{2, \alpha}$ norm) to $\mathfrak{c}$. Then there is a unique strongly asymptotically Einstein metric $g^{\prime}$ which is close to the strongly asymptotically Einstein metric $\tilde{g}^{\prime}$ with conformal infinity data $\mathfrak{c}^{\prime}$ constructed in $\$ 6.2$.

Proof. Let $\tilde{g}^{\prime}$ be the strongly asymptotically Einstein metric constructed in $\$ 6.2$ with conformal infinity data $\mathfrak{c}^{\prime}$. Write $g^{\prime}=\tilde{g}^{\prime}+k$, where

$$
k \in x_{1}^{v} x_{2}^{v} \Lambda_{\mathrm{ph}}^{2, \alpha}\left(M, \operatorname{Sym}^{2}\left(T^{*} M\right)\right) .
$$

Write the gauged Einstein equation as

$$
N^{\tilde{g}^{\prime}}(k)=\operatorname{Ric}^{\tilde{g}^{\prime}+k}+\left(\tilde{g}^{\prime}+k\right)+\left(\delta^{\tilde{g}^{\prime}+k}\right)^{*} B^{\tilde{g}^{\prime}}(k)=0 .
$$

The linearization at $k=0$ is the generalized Laplacian $L^{\tilde{g}^{\prime}}$. 
We see that

$$
N^{\tilde{g}^{\prime}}: x_{1}^{\nu_{1}} x_{2}^{\nu_{2}} \Lambda_{\mathrm{ph}}^{2, \alpha}\left(M, \operatorname{Sym}^{2}\left(T^{*} M\right)\right) \rightarrow x_{1}^{\nu_{1}} x_{2}^{\nu_{2}} \Lambda_{\mathrm{ph}}^{0, \alpha}\left(M, \operatorname{Sym}^{2}\left(T^{*} M\right)\right)
$$

is a $\mathscr{C}^{1}$ map from a neighbourhood of 0 in the domain space, and that $L^{\tilde{g}^{\prime}}$ is a bounded linear map between these same two spaces. Moreover, from our construction of the metric $\tilde{g}^{\prime}$, one has

$$
N^{\tilde{g}^{\prime}}(0) \in x_{1}^{\nu_{1}} x_{2}^{\nu_{2}} \Lambda_{\mathrm{ph}}^{0, \alpha}\left(M, \operatorname{Sym}^{2}\left(T^{*} M\right)\right)
$$

According to Theorem 44, if $\mathfrak{c}^{\prime}$ is sufficiently close to $\mathfrak{c}$, the linearization $L^{\tilde{g}^{\prime}}$ is an isomorphism. Furthermore, the norm of its inverse is bounded away from zero, uniformly as $\mathfrak{c}^{\prime} \rightarrow \mathfrak{c}$, and the norm of $N^{\tilde{g}^{\prime}}(0)$ tends to 0 . The inverse function theorem implies that there is a unique solution $k$ to $N^{\tilde{g}^{\prime}}(k)=0$ with $k$ near 0 .

\section{Appendix. Osculating quaternionic coordinates}

In this brief appendix we prove Lemma 6. This uses an idea close to that used to find normal coordinates in Riemannian geometry, and should be a general fact for all the so called 'parabolic geometries'.

Fix a quaternionic contact structure on $Y^{4 m-1}$ and any metric in this conformal class on the distribution $\mathscr{D}$. We use the Tanaka-Webster type connection $\nabla$ from [2, Chapter 2] (see also $[6,7]$ for the special case of dimension 7). The contact distribution $\mathscr{D}$ is the kernel of three 1 -forms $\left(\eta_{1}, \eta_{2}, \eta_{3}\right)$, and has a privileged supplementary subspace generated by three 'Reeb vectors fields' $R_{1}, R_{2}, R_{3}$ which are uniquely specified by the conditions $\eta_{i}\left(R_{j}\right)=\delta_{i j}$ and $\left.\left(i_{R_{i}} d \eta_{j}+i_{R_{j}} d \eta_{i}\right)\right|_{\mathscr{D}}=0$ (in dimension 7 a weaker condition is placed on these). This connection satisfies:

- $\nabla$ preserves the distribution $\mathscr{D}$ and the quaternionic structure on $\mathscr{D}$;

- the torsion $T^{\nabla}$ of two horizontal vectors $X_{1}, X_{2} \in \mathscr{D}$ is given by

$$
T_{X_{1}, X_{2}}^{\nabla}=\sum_{k=1}^{3} d \eta_{k}\left(X_{1}, X_{2}\right) R_{k}
$$

However, since these conditions only place restrictions on the derivatives in horizontal directions, the connection is not unique and different extensions are possible.

Now fix a point $p \in Y$. We may assume that the $R_{i}$, and hence also the $\eta_{i}$, are parallel at $p$, i.e.

$$
\nabla R_{i}(p)=0, \quad i=1,2,3 .
$$

The connection determines the exponential map in horizontal directions,

$$
\exp _{p}: \mathscr{D}_{p} \rightarrow Y
$$


by solving the differential equation $\nabla_{\dot{c}} \dot{c}=0, c(0)=p, \dot{c}(0)=X$, and setting $\exp _{p}(X)$ $=c(1)$. Linear coordinates $\left(y_{1}, \ldots, y_{4 m-4}\right)$ on $\mathscr{D}_{p}$ give a coordinate system on the image $S$ of a small ball by $\exp _{p}$. Then, at $p$ one has

$$
\nabla_{\partial_{y_{i}}} \partial_{y_{j}}+\nabla_{\partial_{y_{j}}} \partial_{y_{i}}=\frac{1}{2}\left(\nabla_{\partial_{y_{i}}+\partial_{y_{j}}}\left(\partial_{y_{i}}+\partial_{y_{j}}\right)-\nabla_{\partial_{y_{i}}-\partial_{y_{j}}}\left(\partial_{y_{i}}-\partial_{y_{j}}\right)\right)=0,
$$

and hence

$$
\nabla_{\partial_{y_{i}}} \partial_{y_{j}}=\frac{1}{2}\left(\nabla_{\partial_{y_{i}}} \partial_{y_{j}}-\nabla_{\partial_{y_{j}}} \partial_{y_{i}}\right)=\frac{1}{2} T_{\partial_{y_{i}}, \partial_{y_{j}}}^{\nabla}=\frac{1}{2} \sum_{k=1}^{3} d \eta_{k}\left(\partial_{y_{i}}, \partial_{y_{j}}\right) R_{k}
$$

In particular, still at $p$,

$$
\partial_{y_{i}} \eta_{k}\left(\partial_{y_{j}}\right)=\eta_{k}\left(\nabla_{\partial_{y_{i}}} \partial_{y_{j}}\right)=\frac{1}{2} d \eta_{k}\left(\partial_{y_{i}}, \partial_{y_{j}}\right),
$$

from which we deduce that for $y \in S$, the projection $X_{i}$ of $\partial_{y_{i}}$ on $\mathscr{D}$ parallel to the Reeb vector fields $R_{1}, R_{2}$ and $R_{3}$ satisfies

$$
X_{i}=\partial_{y_{j}}-\frac{1}{2} \sum_{k=1}^{3} y_{i} d \eta_{k}\left(\partial_{y_{i}}, \partial_{y_{j}}\right) R_{k}+O\left(|y|^{2}\right) .
$$

This can be interpreted as saying that if we write the standard quaternionic contact structure $\Theta_{0}$ in coordinates $\left(\sigma_{j}, y_{i}\right)$ on the Heisenberg group, and denote by $X_{i}^{0}$ the standard horizontal vector fields as in (3.4) and (3.5), then along $S$ one has

$$
\eta=\Theta_{0}+O\left(|y|^{2}\right), \quad X_{i}=X_{i}^{0}+O\left(|y|^{2}\right) .
$$

Choosing transverse coordinates $\left(\sigma_{1}, \sigma_{2}, \sigma_{3}\right)$ so that $\partial_{\sigma_{i}}=R_{i}$ along $S$, we get the same result in a neighborhood of $p$ with an error term $O\left(|y|^{2}+|\sigma|\right)$.

Acknowledgments. The authors wish to thank the referee for a very careful reading of the manuscript and several helpful comments which led to an improvement of the exposition.

O.B. was supported by CNRS-USA program \# 3332. R.M. was supported by the NSF under Grant DMS-0505709.

\section{References}

[1] Besse, A. L.: Einstein Manifolds. Springer, Berlin (1987) Zbl 0613.53001 MR 0867684

[2] Biquard, O.: Métriques d'Einstein asymptotiquement symétriques. Astérisque 265 (2000); English translation: SMF/AMS Texts Monogr. 13 (2006) Zbl 0967.53030 MR 1760319

[3] Biquard, O.: Métriques autoduales sur la boule. Invent. Math. 148, 545-607 (2002) Zbl 1040.53061 MR 1908060

[4] Biquard, O. (ed.): AdS/CFT Correspondence: Einstein Metrics and Their Conformal Boundaries. IRMA Lectures in Math. Theor. Phys. 8, Eur. Math. Soc., Zürich (2005) Zbl 1062.81002 MR 2160864 
[5] Chruściel, P. T., Delay, E., Lee, J. M., Skinner, D. N.: Boundary regularity of conformally compact Einstein metrics. J. Differential Geom. 69, 111-136 (2005) Zbl 1088.53031 MR 2169584

[6] Duchemin, D.: Géométrie quaternionienne en basses dimensions. PhD thesis, Institut de Recherche Mathématique Avancée, Univ. Louis Pasteur, Strasbourg (2004) MR 2152238

[7] Duchemin, D.: Quaternionic contact structures in dimension 7. Ann. Inst. Fourier (Grenoble) 56, 851-885 (2006) Zbl 1122.53025 MR 2266881

[8] Epstein, C. L., Melrose, R. B.: Shrinking tubes and the $\bar{\partial}$-Neumann problem. Preprint

[9] Epstein, C. L., Melrose, R. B., Mendoza, G. A.: Resolvent of the Laplacian on strictly pseudoconvex domains. Acta Math. 167, 1-106 (1991) Zbl 0758.32010 MR 1111745

[10] Graham, C. R.: Volume and area renormalizations for conformally compact Einstein metrics. In: Proc. 19th Winter School "Geometry and Physics" (Srní, 1999), Rend. Circ. Mat. Palermo (2) Suppl. 63, 31-42 (2000) Zbl 0984.53020 MR 1758076

[11] Graham, C. R., Lee, J. M.: Einstein metrics with prescribed conformal infinity on the ball. Adv. Math. 87, 186-225 (1991) Zbl 0765.53034 MR 1112625

[12] Guillarmou, C.: Meromorphic properties of the resolvent on asymptotically hyperbolic manifolds. Duke Math. J. 129, 1-37 (2005) Zbl 1099.58011 MR 2153454

[13] Helliwell, D.: Boundary regularity for conformally compact Einstein metrics in even dimensions. Comm. Partial Differential Equations 33, 842-880 (2008) Zbl 1145.53037 MR 2424380

[14] Huang, H. C.: The resolvent and scattering operator on near product-hyperbolic spaces. PhD thesis, Stanford Univ. (2006) MR 2709789

[15] Lee, J. M.: Fredholm operators and Einstein metrics on conformally compact manifolds. Mem. Amer. Math. Soc. 183, no. 864 (2006) Zbl 1112.53002 MR 2252687

[16] Lee, J. M., Melrose, R.: Boundary behaviour of the complex Monge-Ampère equation. Acta Math. 148, 159-192 (1982) Zbl 0496.350420496.35042

[17] Mazzeo, R.: The Hodge cohomology of a conformally compact metric. J. Differential Geom. 28, 309-339 (1988) Zbl 0656.53042 MR 0961517

[18] Mazzeo, R.: Elliptic theory of differential edge operators. I. Comm. Partial Differential Equations 16, 1615-1664 (1991) Zbl 0745.58045 MR 1133743

[19] Mazzeo, R.: Regularity for the singular Yamabe problem. Indiana Univ. Math. J. 40, 12771299 (1991) Zbl 0770.53032 MR 1142715

[20] Mazzeo, R. R., Melrose, R. B.: Meromorphic extension of the resolvent on complete spaces with asymptotically constant negative curvature. J. Funct. Anal. 75, 260-310 (1987) Zbl 0636.58034 MR 0916753

[21] Mazzeo, R., Vasy, A.: Resolvents and Martin boundaries of product spaces. Geom. Funct. Anal. 12, 1018-1079 (2002) Zbl 1031.58020 MR 1937834

[22] Mazzeo, R., Vasy, A.: Analytic continuation of the resolvent of the Laplacian on SL(3)/SO(3). Amer. J. Math. 126, 821-844 (2004) Zbl 1060.58020 MR 2075483

[23] Mazzeo, R., Vasy, A.: Analytic continuation of the resolvent of the Laplacian on symmetric spaces of noncompact type. J. Funct. Anal. 228, 311-368 (2005) Zbl 1082.58029 MR 2175410

[24] Mazzeo, R., Vasy, A.: Scattering theory on SL(3)/SO(3): connections with quantum 3-body scattering. Proc. London Math. Soc. 94, 545-593 (2007) Zbl 1117.43009 MR 2325313

[25] Melrose, R. B.: Calculus of conormal distributions on manifolds with corners. Int. Math. Res. Notices 1992, no. 3, 51-61 Zbl 0754.58035 MR 1154213

[26] Shubin, M. A.: Pseudodifferential Operators and Spectral Theory. 2nd ed., Springer, Berlin (2001) Zbl 0980.35180 MR 1852334

[27] Taylor, M. E.: Partial Differential Equations. II. Appl. Math. Sci. 116, Springer, New York (1996) Zbl 0869.35003 MR 1395149 